\title{
Dimensional reduction for D3-brane moduli
}

\author{
Brad Cownden, ${ }^{a}$ Andrew R. Frey, ${ }^{a, b}$ M.C. David Marsh ${ }^{c}$ and Bret Underwood ${ }^{d}$ \\ ${ }^{a}$ Department of Physics \& Astronomy, University of Manitoba, \\ Winnipeg, Manitoba R3T 2N2, Canada \\ ${ }^{b}$ Department of Physics, University of Winnipeg, \\ Winnipeg, Manitoba R3B 2E9, Canada \\ ${ }^{c}$ Department of Applied Mathematics and Theoretical Physics, University of Cambridge, \\ Cambridge, CB3 0WA, U.K. \\ ${ }^{d}$ Department of Physics, Pacific Lutheran University, \\ Tacoma, WA 98447, U.S.A. \\ E-mail: cowndenb@myumanitoba.ca, a.frey@uwinnipeg.ca, \\ m.c.d.marsh@damtp.cam.ac.uk, bret.underwood@plu.edu
}

ABSTRACT: Warped string compactifications are central to many attempts to stabilize moduli and connect string theory with cosmology and particle phenomenology. We present a first-principles derivation of the low-energy $4 \mathrm{D}$ effective theory from dimensional reduction of a D3-brane in a warped Calabi-Yau compactification of type IIB string theory with imaginary self-dual 3-form flux, including effects of D3-brane motion beyond the probe approximation, and find the metric on the moduli space of brane positions, the universal volume modulus, and axions descending from the 4-form potential. As D3-branes may be considered as carrying either electric or magnetic charges for the self-dual 5-form field strength, we present calculations in both duality frames. Our results are consistent with, but extend significantly, earlier results on the low-energy effective theory arising from D3branes in string compactifications.

KEYWORDS: Flux compactifications, D-branes

ARXIV EPRINT: 1609.05904 


\section{Contents}

1 Introduction 1

2 Background and fluctuations $\quad 4$

2.1 Flux compactifications 4

2.2 Dimensional reduction and the kinetic action 5

$\begin{array}{lll}2.3 & \text { Equations of motion with D3-brane sources } & 7\end{array}$

3 Electric D3-brane couplings $\quad 9$

$\begin{array}{lll}3.1 & \text { D3-brane fluctuations in electric formalism } & 10\end{array}$

$\begin{array}{lll}\text { 3.1.1 Summary } & 12\end{array}$

$\begin{array}{lll}3.2 & \text { Kähler moduli in electric components } & 12\end{array}$

$\begin{array}{lll}3.2 .1 & \text { Summary } & 15\end{array}$

$\begin{array}{lll}3.3 & 4 \mathrm{D} \text { effective action in electric formalism } & 15\end{array}$

$\begin{array}{lll}\text { 3.3.1 Contributions to effective action } & 15\end{array}$

$\begin{array}{lll}\text { 3.3.2 Scalar axions and kinetic action } & 17\end{array}$

$\begin{array}{lll}3.3 .3 & \text { Summary } & 19\end{array}$

4 Magnetic D3-brane couplings $\quad 19$

4.1 D3-brane fluctuations in magnetic formalism 20

$\begin{array}{lll}\text { 4.1.1 Summary } & 21\end{array}$

4.2 Non-trivial Bianchi identities, field redefintions, and EOM 21

4.2.1 Field redefinition in background 3-Form 22

4.2.2 Field redefinition for D3-brane source 23

4.2.3 The brane EOM in the magnetic picture 26

$\begin{array}{lll}4.2 .4 & \text { Summary } & 30\end{array}$

4.3 4D effective action in magnetic formalism 30

4.3.1 Kähler moduli in magnetic components 31

$\begin{array}{lll}4.3 .2 & \text { Effective action } & 32\end{array}$

$\begin{array}{lll}\text { 4.3.3 Summary } & 35\end{array}$

5 Discussion $\quad 35$

$\begin{array}{ll}\text { A Conventions } & 36\end{array}$

$\begin{array}{ll}\text { B Bitensors, expansions, and Green's functions } & 37\end{array}$

C Linearized equations of motion $\quad 40$

C.1 Electric formalism equations of motion 40

$\begin{array}{lll}\text { C.1.1 Einstein equations } & 40\end{array}$

C.1.2 Form flux EOM 42

C.1.3 Brane EOM 43 
C.2 Magnetic equations of motion 44

$\begin{array}{lll}\text { C.2.1 Einstein equations } & 44\end{array}$

C.2.2 Form flux EOM 45

$\begin{array}{lll}\text { C.2.3 Brane EOM } & 46\end{array}$

$\begin{array}{ll}\text { D Kähler potential and kinetic action } & 47\end{array}$

\section{Introduction}

In any theory with extra dimensions, a first step towards understanding its dynamics is to construct a low energy, 4-dimensional effective theory. In such an effective description, the dynamical degrees of freedom arise as particular fluctuations of the fields present in the higher dimensions. As a classic example, 5-dimensional gravity on a circle gives rise at low energies to a 4-dimensional effective theory with gravity, a $\mathrm{U}(1)$ gauge field, and a scalar, all of which arise as fluctuations of the bulk 5-dimensional metric. In string theory, the low energy limit of compactification also leads to a 4-dimensional effective theory in which the 4-dimensional degrees of freedom arise from fluctuations of the original 10-dimensional fields. These can include bulk fields such as the metric and $p$-form gauge fields, as well as localized sources like branes. The details of the effective theory depend on the details of the compact space and other fields of the higher-dimensional background, and much work has gone into deriving these effective theories (see [1-4] for example). Typically, these effective theories are derived by dimensionally reducing the higher dimensional action for some particular ansatz of the higher dimensional fields (specifically a zero mode of the appropriate differential operator on the extra dimensions). However, as we emphasize, care must be taken that the ansatz chosen is a consistent solution to the higher-dimensional equations of motion.

One set of essential ingredients in string compactifications are the dynamics of localized sources such as D-branes. D-branes arise in string compactifications as sources of StandardModel-like fields [5], supersymmetry-breaking uplifiting [6], and sources of cosmic inflation (as in [7]; see also the reviews [8,9]). The 4-dimensional effective theory for D-branes is commonly obtained by dimensional reduction of the localized Dirac-Born-Infeld and Chern-Simons actions in the probe approximation. However, the probe approximation cannot address several related conceptual problems.

As an example, consider a $\mathrm{D} p$-brane in an internal space described by the coordinates $\left\{y^{m}\right\}$, so that the brane spans 4-dimensional spacetime $\left\{x^{\mu}\right\}$ embedded with coordinates $Y$. Let us write the D-brane embedding as a constant reference value plus a spacetimedependent fluctuation $Y=Y_{0}+\delta Y(x)$. In the probe approximation, the D-brane degrees of freedom are described by the spacetime-dependent transverse coordinates $\partial_{\mu} Y=\partial_{\mu} \delta Y(x)$ and are independent fluctuations in 10-dimensions. However, when the brane is coupled to gravity it is always possible to make a spacetime-dependent coordinate redefinition (diffeomorphism) of the transverse coordinates $y \rightarrow \tilde{y}, Y \rightarrow \tilde{Y}$ so hypersurfaces of constant 

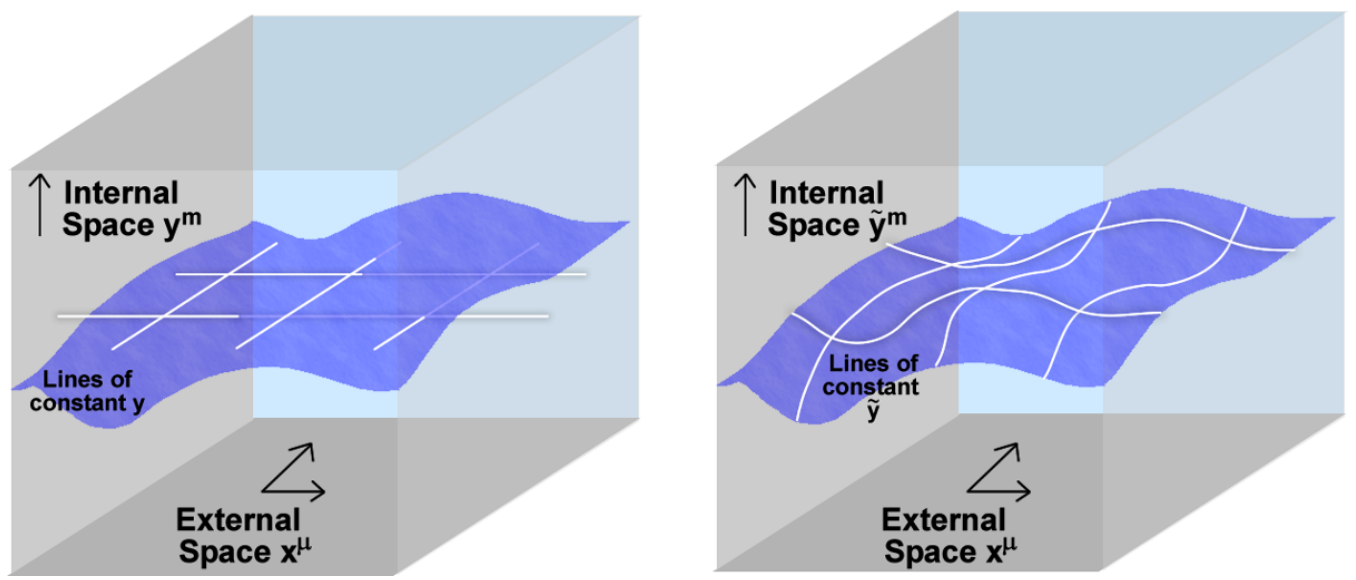

Figure 1. A spacetime-dependent fluctuation of a brane can be described by its transverse coordinates (left). However, it is also possible to redefine the coordinates in a spacetime-dependent way, so the brane fluctuation is "gauged away" (right). In this case, the brane fluctuation is "eaten" by the metric.

$\tilde{y}$ coincide with the worldvolume of the D-brane, as in figure 1 . The brane embedding coordinates no longer encode the D-brane degrees of freedom, since they are now, by definition, spacetime-independent $\partial_{\mu} \tilde{Y}=0$. The D-brane degrees of freedom have been "eaten" by the metric, and the effective theory for the D-brane degrees of freedom now arises not from the localized sources but from the dynamics of the metric. It is interesting to note that this lack of diffeomorphism invariance of the D-brane transverse coordinates implies that the true diffeomorphism invariant degrees of freedom describing the motion of D-branes are a combination of the transverse coordinates and the metric - a combination of open and closed string sectors.

One might think that the simplest resolution is just to fix the gauge such that all of the degrees of freedom are found in the transverse coordinates and not in the metric. Unfortunately, the linearized higher dimensional equations of motion (EOM) do not allow one to choose a gauge with vanishing metric fluctuations. As an example, consider a scalar field in a 4-dimensional homogeneous and isotropic cosmological background. Fluctuations of the scalar field about a time-dependent background $\phi=\phi_{0}(t)+\delta \phi(t, x)$ can be removed by an appropriate time redefinition $t \rightarrow \tilde{t}(t, x)$ so that the true gauge-invariant degree of freedom is a combination of scalar field and metric fluctuations [10]. It is not possible to work in a fixed gauge in which the fluctuation only appears in the scalar field, setting the metric fluctuations to zero, because there is a contribution to the off-diagonal Einstein equations of the form

$$
0=G_{0 i}-\kappa_{4}^{2} T_{0 i}=-\frac{1}{2} \kappa_{4}^{2} \dot{\phi}_{0} \partial_{i} \delta \phi
$$

These off-diagonal Einstein equations act as non-dynamical constraints on the fluctuations, and must be solved for a consistent dynamical description of the scalar perturbations, even at linear order. As is well known, the correct ansatz for scalar cosmological perturbation theory includes simultaneous fluctuations of the metric and scalar field, allowing one to 
consistently solve the constraint equations. The metric fluctuations then play an important role in determining the equation of motion for the dynamics of the scalar degree of freedom.

Similarly, there is no consistent gauge in which the metric does not contain any of the degrees of freedom for the D-brane. In particular, an ansatz with a static metric but spacetime-dependent D-brane fails in two ways even at linear order. First, the change in brane position backreacts on the 10-dimensional metric (specifically the warp factor). In addition, there is a contribution to the off-diagonal 10-dimensional Einstein equations through the energy-momentum tensor of the form

$$
0=G_{\mu m}-\kappa_{10}^{2} T_{\mu m}=-\kappa_{10}^{2} T_{3} \partial_{\mu} Y_{m}(x) \delta^{6}(y, Y) .
$$

These constraints on the D-brane motion arise from the required gauge-fixing described above, much like the Gauss law constraint of electromagnetism or the Hamiltonian and momentum constraints of gravitation. As a result, the probe-brane effective theory based on the D-brane degrees of freedom residing entirely in the localized transverse coordinates is incomplete. The appropriate 10-dimensional ansatz will require including parts of the Dbrane degrees of freedom in both the metric and the transverse coordinates, as we will see.

One concern with moving beyond the probe approximation is that inserting the backreacted brane solution into the effective action could cause a "self-energy" problem in which the effective action diverges. We explicitly show in section 3.3 that when one carefully performs a dimensional reduction which solves the constraint equations the resulting effective action does not contain any such divergent self-energy terms.

In this paper, as a test case and for concreteness, we will focus on the dynamics of D3-branes in the type IIB backgrounds of the form given in [11-14] (commonly called GKP compactifications). In these backgrounds, the positions of D3-branes (along with metric Kähler moduli and various axions) are moduli, and the effective theory is described by a 4D supergravity (possibly with spontaneous supersymmetry breaking). As a result, the effective theory arises from a Kähler potential (see for example $[1,7,15]$ ). One challenge for the construction of an effective theory in GKP backgrounds is that the metric becomes a warped product between the internal and external spaces, complicating the identification of the degrees of freedom. The supersymmetry of the background along with the fact that scaling the warp factor can be removed with a 10D diffeomorphism has allowed [16-19] to derive many aspects of the effective theory without a direct dimensional reduction.

However, to work with generic warped compactifications without so much structure, it is necessary to develop techniques for dimensional reduction in warped metrics. For example, [20] showed that the dilaton and volume modulus are identified under diffeomorphisms for warped backgrounds with a dilaton profile. Fortunately, work on warped effective field theory (see [20-26]) has led to some useful formalisms [20, 23] for constructing and analyzing fluctuations of these 10-dimensional backgrounds and their effective theories. In [25], the effective theory for the universal volume modulus and its associated axion was derived for GKP compactifications, while [26] improved and extended this analysis to include the rest of the axion sector. ${ }^{1}$ Our first-principles approach is to choose an ansatz in a fixed

\footnotetext{
${ }^{1} \mathrm{~A}$ recent analysis in [19] shows that the supersymmetric formalism [16-18] in fact agrees with direct dimensional reduction [26] in detail for GKP compactifications.
} 
gauge (for diffeomorphisms and the supergravity form fields) and ensure that it solves all the constraints of the 10D theory even when the 4D fluctuation is off shell.

An initial attempt [27] to derive the effective theory for D3-branes in these backgrounds was unable to solve the constraint equations coming from the higher-dimensional EOM and was unable to derive the terms (including mixing terms) necessary to construct the Kähler potential and effective theory. We will use the formalism and techniques developed in $[20,26]$ to obtain an ansatz which does solve all of the higher dimensional EOM and to derive all the necessary terms in the effective theory. It is not essential that the D3-brane we study in this paper is localized in a strongly warped region: the effects discussed above are inherent to the D-brane's interaction with the background, and thus will be present regardless of the strength of the warping at the location of the D3-brane.

In fact, we will present two calculations of this effective action. The self-duality of the type IIB five-form causes the action to vanish if all the components of the $10 \mathrm{D}$ covariant tensor are included. Following [14], we discard half the components of the five-form in the dimensional reduction of the action. If we keep components with legs mostly along the external dimensions, the D3-brane carries an electric charge for the five-form; keeping the complementary set of components leads the D3-brane to carry magnetic monopole charge. In the former case, axions of the 4D theory arise as 2-form degrees of freedom, and cross terms between the brane position and axion descend from the brane's Wess-Zumino action. Meanwhile, in the latter case, we solve the nontrivial Bianchi identity by finding a field redefinition that gives the 5 -form field strength an explicit dependence on the brane position, and cross terms in the kinetic action arise directly through backreaction of the brane on the field strength. This procedure is related to Dirac's original proposal for a Lagrangian describing the coupling of magnetic monopoles and Maxwell fields [28]; the relationship of our procedure to Dirac's theory and its generalization to branes will appear in a forthcoming paper [29].

The plan of our paper is as follows. In section 2, we review the background compactification, the procedure for dimensional reduction, and the EOM of type IIB supergravity with D3-branes. We then give the dimensional reduction in the version of the theory in which the D3-brane carries electric charge for the five-form in 3, followed by the analogous calculation for the magnetically charged D3-brane in section 4. In these two sections, we provide a short summary at the end of each subsection encapsulating the important results as an aid to the reader. We close with a discussion; conventions and formalism are found in the appendices.

\section{$2 \quad$ Background and fluctuations}

\subsection{Flux compactifications}

To set our conventions, we work in the bosonic sector of type IIB string theory as described by the low-energy supergravity (SUGRA)

$$
\begin{aligned}
S_{\text {IIB }}= & \frac{1}{2 \kappa_{10}^{2}} \int d^{10} x \sqrt{-g}\left(\mathcal{R}-\frac{\partial_{M} \tau \partial^{M} \bar{\tau}}{2(\operatorname{Im} \tau)^{2}}\right)-\frac{1}{2 \kappa_{10}^{2}} \int\left[\frac{G_{3} \wedge \star \bar{G}_{3}}{12 \operatorname{Im} \tau}+\frac{1}{4} \tilde{F}_{5} \wedge \star \tilde{F}_{5}\right. \\
& \left.+\frac{i}{4 \operatorname{Im} \tau} C_{4} \wedge G_{3} \wedge \bar{G}_{3}\right]+S_{\text {loc }},
\end{aligned}
$$


where $\mathcal{R}$ is the ten-dimensional (10D) Ricci scalar, and $\kappa_{10}$ is the 10D Newton's constant. We have defined the axio-dilaton $\tau=C_{0}+i e^{-\phi}$, combined the 3 -forms $F_{3}=d C_{2}$ and $H_{3}=d B_{2}$ into the complex 3-form $G_{3}=F_{3}-\tau H_{3}$, and defined the five-form field strength as $\tilde{F}_{5}=d C_{4}-C_{2} \wedge H_{3}$, which is constrained to be self-dual: $\tilde{F}_{5}=\star \tilde{F}_{5} . S_{\text {loc }}$ is the action for all the local objects, including D3-branes.

The background fields of GKP compactifications take the form

$$
\begin{aligned}
& d s_{10}^{2}=e^{2 A^{(0)}(y)} \hat{\eta}_{\mu \nu} d x^{\mu} d x^{\nu}+e^{-2 A^{(0)}(y)} \tilde{g}_{m n} d y^{m} d y^{n}, \\
& \tilde{F}_{5}^{(0)}=\hat{\epsilon} \wedge \tilde{d} e^{4 A^{(0)}}+\tilde{\star} \tilde{d} e^{-4 A^{(0)}}, \quad \tilde{\star} G_{3}^{(0)}=i G_{3}^{(0)},
\end{aligned}
$$

where $\left\{x^{\mu}\right\}$ spans 4-dimensional (4D) spacetime and $\left\{y^{m}\right\}$ spans the internal dimensions. The unwarped metrics $\hat{\eta}_{\mu \nu}, \tilde{g}_{m n}$ are respectively Minkowski and Calabi-Yau (CY). We denote objects constructed with respect to $\hat{\eta}_{\mu \nu}$ with a hat and those with respect to $\tilde{g}_{m n}$ with a tilde. The three-form field strength is harmonic, and the background warp factor, $A^{(0)}(y)$, obeys a Poisson equation

$$
\tilde{\nabla}^{2} e^{-4 A^{(0)}}=-\frac{g_{s}}{2}\left|G_{3}^{(0)}\right|^{\tilde{2}}-2 T_{3} \kappa_{10}^{2} \tilde{\delta}^{6}\left(y, Y^{(0)}\right)-\cdots,
$$

where $\cdots$ are other local sources including more D3-branes (which also contribute delta function sources), D7-branes, and negative charge and tension orientifold O3- and O7planes. Although only a single D3-brane (located at $Y$ ) is considered here and throughout, the extension to multiple, non-interacting branes of this type is trivial. Furthermore, we work in the orientifold limit where four D7-branes are coincident with each O7-plane for simplicity, although we expect our results to generalize straightforwardly to F-theory.

A nontrivial $G_{3}^{(0)}$ also stabilizes moduli of the compactification, generically including all the complex structure moduli of $\tilde{g}_{m n}$ and the axiodilaton $\tau$. (See [26] for a more complete review of the effects of the flux on moduli stabilization.) As a result, we assume that the complex structure and $\tau$ are constant. In addition, the background is supersymmetric at the classical level when $G_{3}^{(0)}$ is $(2,1)$ in the CY complex coordinates; ${ }^{2}$ if the flux breaks supersymmetry, it does so spontaneously [21], and the effective theory can be described by 4D SUGRA.

With $\tau$ assumed to be constant, we can write $G_{3}=d A_{2}$ in terms of a complex potential $A_{2}=B_{2}-\tau C_{2}$. In this case, it is common to redefine $C_{4}$ to set $\tilde{F}_{5}=d C_{4}+\left(i g_{s} / 4\right)\left(A_{2} \wedge\right.$ $\left.\bar{G}_{3}-\bar{A}_{2} \wedge G_{3}\right)$. We will use this definition for $\tilde{F}_{5}$ henceforth.

\subsection{Dimensional reduction and the kinetic action}

To determine the effective 4D theory, we must first decompose the 10D fields into orthogonal modes, each of which corresponds to a $4 \mathrm{D}$ degree of freedom. In a product space compactification, these modes are simply the eigenfunctions of some second order differential operator on the compact space (for example, the Laplacian for a scalar or the Hodge-de Rham operator for a form field). However, the constraints described in the introduction complicate matters somewhat for warped compactifications. We are particularly interested

\footnotetext{
${ }^{2}$ And primitive, which is automatically satisfied on a generic CY.
} 
in clasically massless moduli, so we consider only those modes that satisfy the massless Klein-Gordon equation in the external $x^{\mu}$ directions. The following sections will describe the structure of these massless modes; here, we will outline the procedure of dimensionally reducing the action once the linearized modes are known, following [26].

To find the two-derivative kinetic action in terms of a metric $G_{a b}(\phi)$ on moduli space, we need find only the action to quadratic order in fluctuations around the background as long as we expand the background around an arbitrary point $\phi_{0}$ in moduli space, we recover the full dependence of the metric on the moduli. While our primary consideration is D3-brane position moduli, we are interested in the metric on the Kähler moduli space of the CY. We therefore also consider the universal volume modulus and axions descending from the 4-form potential (which are partners of the Kähler metric moduli). Because the $4 \mathrm{D}$ effective theory is a SUGRA, the moduli space is naturally described in terms of holomorphic coordinates, and the metric is defined in terms of a Kähler potential.

This quadratic action can be written in terms of the first-order fluctuations and the linearized EOM, as demonstrated in [26] (and used implicitly in [24]). Specifically, for type IIB SUGRA, we can write

$$
\begin{aligned}
S= & \frac{1}{4 \kappa_{10}^{2}} \int d^{10} x \sqrt{-g} \delta g^{M N} \delta E_{M N}+\frac{1}{4 \kappa_{10}^{2}} \int\left(\delta C_{4} \wedge \delta E_{6}+\frac{g_{s}}{2} \delta A_{2} \wedge \delta \bar{E}_{8}+\frac{g_{s}}{2} \delta \bar{A}_{2} \wedge \delta E_{8}\right) \\
& +\frac{T_{3}}{2} \int d^{10} x \sqrt{-g} \int d^{4} \xi \sqrt{-\gamma} \delta X^{M} \delta E_{M I},
\end{aligned}
$$

where $\delta E_{m n}$ is the linearized Einstein equation, $\delta E_{6}$ the linearized EOM for the 4 -form, $\delta E_{8}$ for the 2-form potential, and $\delta E_{M}$ for the D3-brane position. The EOM for the worldvolume metric $\gamma_{a b}$ (as described below) are higher-order (and constraints), so they do not contribute (though $\gamma_{a b}$ should be evaluated on the solution). Note that we have labeled the brane embedding coordinate $X^{M}(\xi)$ with a slashed index to indicate that tensors at that point do not contract with tensors at a general point $x^{M}$ in spacetime, and this term is integrated over the brane worldvolume coordinates as well as spacetime. Furthermore, these are the EOM as they directly arise from the variation of the action; in particular, the equations of type IIB SUGRA are often reorganized to remove terms proportional to $E_{6}$ from $E_{8}$.

There are also two subtleties to note. First, there is not actually a covariant action in 10D that respects the self-duality of the 5 -form field strength, ${ }^{3}$ so (2.4) includes only some of the components of $C_{4}$ and the corresponding EOM. We describe our approach to this subtlety in section 2.3 below. Second, [26] arrives at the action (2.4) after an integration by parts; in some cases, there are terms in the action which are quadratic in fluctuations but contribute no terms to the linearized EOM (for example, the axionic coupling $\theta F^{2}$ where the field strength is first order). These terms must be added separately to the quadratic action, as we will see in section 3.3.

The action (2.4) should also be understood in a fixed gauge (for diffeomorphisms, form gauge transformations, and worldvolume reparameterization). We choose a gauge in

\footnotetext{
${ }^{3}$ For the usual fields of IIB SUGRA; Sen [30] has recently (during preparation of this manuscript) described a covariant action with different field content.
} 
which the underlying CY metric $\tilde{g}_{m n}$ of the internal directions does not fluctuate (and form potential gauge as described below); for D3-branes with internal positions $Y^{\eta h}$ that vary slowly over the external spacetime, we find it convenient to use a static gauge $\xi^{a}=\delta_{\mu}^{a} X^{\mu}(\xi)$ so that fluctuations of $X^{\mu}$ are gauged away (with $\left|\partial_{a} Y^{\not h}\right|$ assumed to be small).

\subsection{Equations of motion with D3-brane sources}

In this section, we summarize the 10-dimensional EOM for type IIB supergravity with D3-brane sources. The bulk SUGRA equations are well-known [31], but the brane sources are less familiar. We therefore simply state the bulk terms but provide a brief derivation of the D3 sources. As in section 2.2, we use slashed indices at the D3-brane position; the parallel propagator $\Lambda_{M}^{M /}(x, X)$ (or its inverse) switches index type for fields evaluated at coincidence (i.e. when multiplied by a delta function).

The variation of the action with respect to the metric, $E_{M N}$, is

$$
E_{M N}=R_{M N}-\frac{1}{2} g_{M N} \mathcal{R}-\left(T_{M N}^{5}+T_{M N}^{3}+T_{M N}^{D 3}+T_{M N}^{l o c}\right),
$$

so that setting $E_{M N}=0$ gives the 10-dimensional Einstein equations. The contributions to the energy-momentum from the 5-form flux (given 5-form self-duality) and background 3-form are

$$
T_{M N}^{5}=\frac{1}{4 \cdot 4 !} \tilde{F}_{M P Q R S} \tilde{F}_{N} P Q R S, \quad T_{M N}^{3}=\frac{g_{s}}{4}\left(G_{(M}^{P Q} \bar{G}_{N) P Q}-g_{M N}|G|^{2}\right) .
$$

The D3-brane coupling to the metric enters in the Dirac-Born-Infeld (DBI) terms in the brane action; if we ignore couplings to the 2-form potentials and world-volume gauge field (justified below), we can replace the usual DBI action for the brane with a classically equivalent Polyakov-like form

$$
S_{D B I}=-\frac{T_{3}}{2} \int d^{10} x \sqrt{-g} \int d^{4} \xi \sqrt{-\gamma}\left[\gamma^{a b} P(g)_{a b}-2\right] \delta^{10}(x, X(\xi)),
$$

where $\gamma_{a b}$ is an independent worldvolume metric which equals the induced metric $P(g)_{a b}=$ $g_{M \perp X} \partial_{a} X^{M /} \partial_{b} X^{\perp / N}$ on shell. Regarding delta functions in curved spacetime, we refer the reader to appendix B. The resulting energy-momentum from D3-branes is then

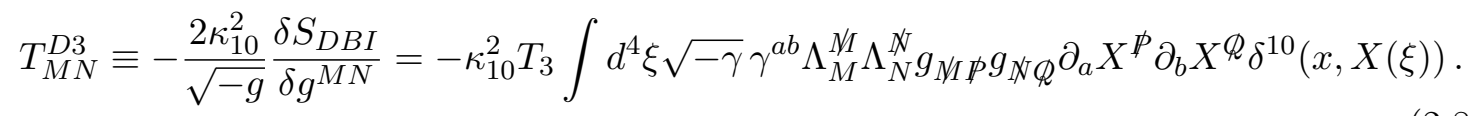

A similar expression leads to the energy-momentum $T_{M N}^{l o c}$ from localized sources other than the mobile D3-brane of interest. For notational convenience, we will often make the parallel propagators implicit, writing for example $g_{M \not p}=\Lambda_{M}^{M} g_{M \not p}$.

Self-duality of $\tilde{F}_{5}$ raises some complications for dimensional reduction at the level of the action. Specifically, evaluating (2.1) on a self-dual 5-form leads to a vanishing kinetic term, whether on or off shell. The prescription we follow is to replace (2.1) by a non-covariant action, retaining only half the components of $\tilde{F}_{5}$ and doubling the coefficient of the $\tilde{F}_{5} \wedge \star \tilde{F}_{5}$ term in the action. We will make two distinct choices for the sets of components to keep: 
the "electric" set with 4 or 3 legs on $x^{\mu}$ and 1 or 2 respectively on $y^{m}$, and the "magnetic" set with 0 or 1 leg on $x^{\mu}$ and 5 or 4 on $y^{m}$ (an equal number of components with 2 on $x^{\mu}$ and 3 on $y^{m}$ fall in each set, but these all vanish for the moduli we consider). The corresponding components of $C_{4}$ for the electric set couple electrically to the D3-brane, whereas the D3-brane is a magnetic source for the magnetic set. ${ }^{4}$ It is worth noting that the components in each set are Hodge dual to the components in the other set.

We begin by considering the theory for the electric components. In this case, the Bianchi identity is trivial $\left(d \tilde{F}_{5}=0\right)$, and the source for $d \star \tilde{F}_{5}$ arises through the D3-brane Wess-Zumino (WZ) action (again ignoring couplings to the 2-form potentials for now)

$$
\begin{aligned}
S_{\mathrm{WZ}} & =\mu_{3} \int d^{10} x \sqrt{-g} \int_{\xi} P\left(C_{4}\right) \delta^{10}(x, X(\xi)) \\
& =\mu_{3} \int d^{4} \xi \sqrt{-\gamma} \int_{10} C_{4} \wedge \star \epsilon_{\|} \delta^{10}(x, X(\xi)),
\end{aligned}
$$

where $\epsilon_{\|}$is the push-forward of the antisymmetric world-volume tensor. We define the push-forward as

$$
\epsilon_{\|}^{M I N \perp P Q} \equiv \epsilon^{a b c d} \partial_{a} X^{M I} \partial_{b} X^{\perp N} \partial_{c} X^{\not P} \partial_{d} X^{\varnothing}
$$

and take parallel propagators to be implicit in the 10D Hodge star. As we will see below, D3-branes which are mutually BPS with the background have charge equal to tension $\mu_{3}=T_{3}$. The resulting 5 -form EOM, including contributions from the 3 -form, is therefore

$$
E_{6}=d \star \tilde{F}_{5}-\frac{i g_{s}}{2} G_{3} \wedge \bar{G}_{3}+2 \kappa_{10}^{2} T_{3} \int d^{4} \xi \sqrt{-\gamma} \star \epsilon_{\|} \delta^{10}(x, X(\xi)),
$$

and vanishes on shell. ${ }^{5}$ Since the 3 -form has at most one leg in the external spacetime, $G_{3}$ contributes only to the EOM and not the Bianchi identity.

The EOM and Bianchi identity for the magnetic components of $\tilde{F}_{5}$ are simply given by exchanging $\tilde{F}_{5} \leftrightarrow \star \tilde{F}_{5}$ in the corresponding equations for the electric components. Therefore, the EOM is $E_{6}=d \star \tilde{F}_{5}$, while the Bianchi identity becomes

$$
0=d \tilde{F}_{5}-\frac{i g_{s}}{2} G_{3} \wedge \bar{G}_{3}+2 \kappa_{10}^{2} T_{3} \int d^{4} \xi \sqrt{-\gamma} \star \epsilon_{\|} \delta^{10}(x, X(\xi)) .
$$

We will consider both (2.11), (2.12) in the static gauge later. As discussed in [21, 25, 26], the nontrivial Bianchi identity (2.12) for the magnetic components means that perturbations of $C_{4}$ as defined in (2.1) and below are not globally defined on the CY manifold. Those references studied the Bianchi identity in the absence of D3-branes and found a field redefinition with a globally-defined 4 -form potential. In terms of the new $C_{4}$ (in the absence of axions descending from $\left.A_{2}\right)$, perturbations of $\tilde{F}_{5}$ are $\delta \tilde{F}_{5}=d \delta C_{4}+\left(i g_{s} / 2\right)\left(\delta A_{2} \bar{G}_{3}^{(0)}-\delta \bar{A}_{2} G_{3}^{(0)}\right)$.

\footnotetext{
${ }^{4}$ Technically, there is a WZ action coupling $C_{4}$ to the brane, but it appears at higher order in spacetime derivatives than we consider.

${ }^{5}$ There is one subtlety with factors of 2 ; when varying the covariant action (2.1), we replace $\mu_{3} \rightarrow \mu_{3} / 2$ in $S_{\text {loc }}$ since the WZ term includes magnetic and electric couplings. This is equivalent to keeping only the electric components of $\tilde{F}_{5}$ and doubling the coefficient of the $\tilde{F}_{5} \star \tilde{F}_{5}$ term as in [14].
} 
We will review this field redefinition and demonstrate the additional redefinition needed for the D3-brane contribution to the Bianchi identity in section 4.

The 3-form is somewhat simpler; the Bianchi identity is trivial $d G_{3}=0$ for constant axio-dilaton $\tau$, and the EOM is

$$
E_{8}=d \star G_{3}+i G_{3} \wedge\left(\tilde{F}_{5}+\star \tilde{F}_{5}\right)+\frac{i}{2} A_{2} \wedge E_{6}+\cdots
$$

with either electric or magnetic components for $\tilde{F}_{5}$. The coefficients of the last two terms are given in keeping with our prescription to double the $\tilde{F}_{5}$ kinetic term. The dots represent brane source terms, which we ignore as discussed below. Since we do not consider axions that descend from $A_{2}$ or their backgrounds, the last term given explicitly in (2.13) will not contribute.

Finally, we need to determine the 10-dimensional D3-brane EOM. Due to the different couplings to the electric and magnetic components of the 5-form, these equations take different forms depending on which version of the theory we consider. Here we present the more familiar electric version and discuss the magnetic version in section 4 below. Using the normalization of (2.4), variation of (2.7), (2.9) gives ${ }^{6}$

$$
\begin{aligned}
& E_{M I}=\left\{\nabla_{a}\left[\left(g_{M \perp X} \partial^{a} X^{\not X}+\frac{1}{6} \frac{\mu_{3}}{T_{3}} \epsilon^{a b c d} C_{M L \perp P Q} \partial_{b} X^{X_{N}} \partial_{c} X^{\not P} \partial_{d} X^{Q}\right)\right]\right.
\end{aligned}
$$

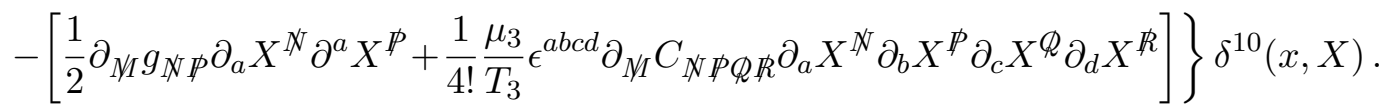

This EOM allows us to set our sign convention for the D3-brane charge (since $\left|\mu_{3}\right|=T_{3}$ ). Consider a static D3-brane $\partial_{a} Y^{\eta h}=0$ in static gauge in the background described above. The $M=\not \mu$ equation becomes $\nabla_{a}\left[\left(1-\mu_{3} / T_{3}\right) \delta^{10}(x, X)\right]=0$, while the $M=\not h$ equation becomes $-4 \partial_{\eta 2} A\left(1-\mu_{3} / T_{3}\right) \delta^{10}(x, X)=0$. Both of these vanish for the choice $\mu_{3}=T_{3}$.

We now justify ignoring the 2-form potential couplings in both the DBI and WZ actions, despite the fact that they appear in the background with nontrivial $G_{3}^{(0)}$. The main point is that the background potentials have completely internal legs and are pulled back to the world volume by two powers of the small derivatives $\partial_{a} Y^{\eta h}$. Furthermore, in a perturbative expansion of the DBI action, the pulled-back potential $P\left(B_{2}\right)$ enters either at second-order, or contracted with the world-volume field strength. These terms are $3 \mathrm{rd}$ and 4th order in fluctuations, so we ignore them. The WZ terms containing $P\left(C_{2}\right)$ and $P\left(B_{2}\right)$ are similarly $3 \mathrm{rd}$ and 4 th order and can also be ignored. Finally, we set the world-volume field strength to zero, since we do not consider vector degrees of freedom.

\section{Electric D3-brane couplings}

As we discussed in section 2.3 above, because of the 10D self-duality of $\tilde{F}_{5}$, a D3-brane can act as either an electric or magnetic source for $C_{4}$. In this section, we consider the "electric" choice as defined above, namely, the choice to keep components of $\tilde{F}_{5}$ with legs

\footnotetext{
${ }^{6}$ The Euler-Lagrange equations also apparently contain terms proportional to $\partial_{M M} \delta^{10}(x, X)$; however, in the variation of the action, these terms vanish upon converting the $X^{M I}$ derivative to an $x^{M}$ derivative and integrating by parts. We therefore do not consider them to be part of the EOM.
} 
mostly along the external spacetime. With this choice, a D3-brane has an electric coupling to $C_{4}$ through the WZ terms in its action.

We begin by first presenting our ansatz for fluctuations in the D3-brane position. As discussed in the introduction, an ansatz for dimensional reduction must satisfy the constraint equations arising from the 10D EOM (2.5), (2.11), (2.14). We will show how our ansatz - which involves the presence of D3-brane degrees of freedom not only as transverse coordinates but also in the metric and 4-form gauge potential - solves these constraint equations and is thus the first known consistent ansatz for the dimensional reduction of transverse D3-brane degrees of freedom. In order to compute the full effective action of the D3-brane, we need to understand how it couples to the massless moduli, such as the volume modulus and $C_{4}$ axions. Dimensional reduction of the volume modulus and axions in GKP compactifications has been studied previously in [25, 26]; however, those articles worked with the other (magnetic) choice for components of $\tilde{F}_{5}$, which leads to a slightly different ansatz for the linearized fluctuations. Thus, we briefly discuss the electric form of linearized fluctuations of these moduli. We will then see that these moduli can all be described by a common "electric" ansatz, which we use to carry out the dimensional reduction of the action to a $4 \mathrm{D}$ effective theory.

\subsection{D3-brane fluctuations in electric formalism}

As discussed in section 2.2, we will consider a D3-brane with embedding coordinates $X^{M /}=\left\{X^{\mu}, Y^{\eta h}\right\}$ with world volume parameterization in static gauge $\xi^{a}=\delta_{\mu h}^{a} X^{\mu}$ (so that fluctuations in $X^{\mu}$ are gauged away) and take small, slowly varying fluctuations of the transverse coordinates of the D3-brane $Y^{\text {hh }}(x)=Y^{(0) \not h}+\delta Y^{\not h}(x)$.

As discussed in the introduction, diffeomorphisms and the constraint equations couple the D3-brane with the metric and 4 -form $C_{4}$, forcing us to go beyond the probe limit for the D3-brane. Thus, fluctuations of the D3-brane transverse position appear in the metric at linear order through the ansatz

$$
d s^{2}=e^{2 \Omega(x)} e^{2 A(x, y)} \hat{\eta}_{\mu \nu} d x^{\mu} d x^{\nu}+2 e^{2 \Omega(x)} e^{2 A(x, y)} \partial_{\mu} B_{m}^{Y}(x, y) d x^{\mu} d y^{m}+e^{-2 A(x, y)} \tilde{g}_{m n} d y^{m} d y^{n},
$$

which uses the same structure as $[25,26]$ for the volume modulus and axions. This is similar to the background (2.2); the additions are spacetime-dependence in the warp factor $A(x, y)$, a "compensator" term containing a 1-form $B_{m}^{Y}(x, y)$ needed to solve the constraints (and which vanishes for spacetime-independent fluctuations), and a Weyl factor $\Omega(x)$ defined by

$$
e^{-2 \Omega(x)} \equiv \frac{1}{\tilde{V}} \int d^{6} y \sqrt{\tilde{g}} e^{-4 A(x, y)} \quad \text { with } \tilde{V} \equiv \int d^{6} y \sqrt{\tilde{g}} .
$$

The Weyl factor is required to diagonalize the $4 \mathrm{D}$ graviton and warped volume fluctuations. We work in a diffeomorphism gauge in which the CY metric $\tilde{g}_{m n}$ is fixed to its background form.

Fluctuations of the D3-brane position also appear in the electric components of the 4-form potential $C_{4}$ through the Weyl factor, warp factor, and compensator

$$
C_{4}=e^{4 \Omega} e^{4 A} \hat{\epsilon}+e^{4 \Omega} e^{4 A} \hat{\star} \hat{d} B_{1}^{Y}
$$


with corresponding 5 -form

$$
\begin{aligned}
\tilde{F}_{5}= & e^{4 \Omega} \hat{\epsilon} \wedge \tilde{d} e^{4 A}+d\left(e^{4 \Omega} e^{4 A_{\hat{\star}} \hat{d} B_{1}^{Y}}\right) \\
& +\left[\tilde{\star} \tilde{d} e^{-4 A}+\star d\left(e^{4 \Omega} e^{4 A_{\hat{\star}} \hat{d} B_{1}^{Y}}\right)-e^{2 \Omega} e^{-4 A_{\tilde{\star}}}\left(\hat{d} B_{1}^{Y} \wedge \tilde{d} e^{4 A}\right)\right] .
\end{aligned}
$$

The terms of (3.4) in square brackets are required in the 10D description of the field strength for self-duality (these are the magnetic components of $\tilde{F}_{5}$ ).

The constraints follow from inserting our ansatz (3.1), (3.2), (3.3), (3.4) into the 10D EOM (2.5), (2.11), and (2.14). We will see that the constraints have specific solutions for $A(x, y), B_{m}^{Y}(x, y)$, and $\Omega(x)$.

First, consider the constraints coming from the non-dynamical terms in the Einstein equations (see appendix C.1). In particular, the $(\mu \nu)$ component yields

$$
\tilde{\nabla}^{2} e^{-4 A(x, y)}=-\frac{g_{s}}{2}\left|G_{3}^{(0)}\right|^{\tilde{2}}-2 \kappa_{10}^{2} T_{3} \tilde{\delta}^{6}(y, Y)-\cdots,
$$

as well as

$$
\tilde{\nabla}^{\tilde{n}} B_{n}^{Y}=e^{-2 \Omega} \delta e^{-4 A}-e^{-4 A} \delta e^{-2 \Omega} .
$$

The first of these, (3.5), promotes the background Poisson equation for the warp factor (2.3) to include first-order contributions from the D3-brane "instantaneously" (i.e., separately at each point $x^{\mu}$ in spacetime):

$$
\begin{aligned}
e^{-4 A^{(0)}(y) \rightarrow e^{-4 A(x, y)}} & =2 \kappa_{10}^{2} T_{3} \tilde{G}\left(y, Y^{(0)}+\delta Y\right)+\cdots \\
& =2 \kappa_{10}^{2} T_{3} \tilde{G}\left(y, Y^{(0)}\right)+2 \kappa_{10}^{2} T_{3} \delta Y^{\eta_{h}} \partial_{\eta_{\imath}} \tilde{G}(y, Y)+\cdots \\
& =e^{-4 A^{(0)}(y)}+2 \kappa_{10}^{2} T_{3} \delta Y^{\eta_{h}} \partial_{\eta_{\imath}} \tilde{G}(y, Y),
\end{aligned}
$$

where the $\cdots$ represent the other contributions to the warp factor due to fluxes and other localized sources (which are smooth at $Y$ ) and $\tilde{G}(y, Y)$ is the biscalar Green's function on the internal CY. ${ }^{7}$ In this sense, our diffeomorphism gauge choice is analogous to the Coulomb gauge describing electromagnetic radiation. Equation (3.7) also shows that the Weyl factor $\Omega(x)$ is independent of the D3-brane moduli at linear order, since

$$
\delta_{Y} e^{-2 \Omega}=\frac{2 \kappa_{10}^{2} T_{3}}{\tilde{V}} \delta Y^{\not h} \int d^{6} y \sqrt{\tilde{g}} \partial_{\eta \downarrow} \tilde{G}(y, Y)=-\frac{2 \kappa_{10}^{2} T_{3}}{\tilde{V}} \delta Y^{\not h} \int d^{6} y \sqrt{\tilde{g}} \tilde{\nabla}_{n} \tilde{G}_{\not h}^{n}=0,
$$

via the relation (B.10) between scalar and tensor Green's functions. Nevertheless, we will keep the Weyl factor in our calculations since it is important for defining the $4 \mathrm{D}$ Einstein frame.

Next, the mixed component of the Einstein equation gives a non-trivial constraint

$$
-\frac{1}{2} \partial_{\mu} \partial_{m} e^{-4 A}+\frac{1}{2} e^{2 \Omega} \partial_{\mu} \tilde{\nabla}^{\tilde{n}}\left(\tilde{d} B_{1}^{Y}\right)_{m n}+\kappa_{10}^{2} T_{3} \tilde{g}_{m \eta} \partial_{\mu} Y^{\hbar} \tilde{\delta}^{6}(y, Y)=0
$$

\footnotetext{
${ }^{7}$ See appendix B for definitions and properties of bitensor Green's functions.
} 
as well as a copy of (3.5) multiplied by $\partial_{\mu} B_{m}^{Y}$. Since $\tilde{\nabla}^{\tilde{n}}\left(\tilde{d} B_{1}\right)_{m n}=-\tilde{\nabla}^{2} B_{m}+\tilde{\nabla}_{m} \tilde{\nabla}^{\tilde{n}} B_{n}$ on the Ricci flat CY, (3.6) and (3.9) yield

$$
\tilde{\nabla}^{2} B_{m}^{Y}=2 \kappa_{10}^{2} T_{3} e^{-2 \Omega} \tilde{Y}_{m} \tilde{\delta}^{6}(y, Y) \text {, where } \tilde{Y}_{m}=\tilde{g}_{m \not h} \delta Y^{\not h} .
$$

The compensator is given by the bivector Green's function

$$
B_{m}^{Y}(x, y)=-2 \kappa_{10}^{2} T_{3} e^{-2 \Omega} \tilde{g}_{m n} \delta Y^{\not p} \tilde{G}_{\not p}^{n}(y, Y) .
$$

The Green's function identity (B.10) implies that $B_{1}^{Y}$ automatically satisfies (3.6).

The EOM for the 5-form flux (2.11), evaluated for this ansatz in (C.20) in detail, also contributes a constraint equation. The source term for (2.11) in static gauge is

$$
\int d^{4} \xi \sqrt{-\gamma} \star \epsilon_{\|} \delta^{10}(x, X(\xi))=\star \epsilon_{\|} \delta^{6}(y, Y(x))=-\left(\tilde{\epsilon}+\hat{d} \tilde{\star} \tilde{Y}_{1}\right) \tilde{\delta}^{6}(y, Y),
$$

and the constraints are another copy of (3.5) and

$$
\hat{d}\left[\tilde{\star} \tilde{d} e^{-4 A}+e^{2 \Omega} \tilde{d} \tilde{\star} \tilde{d} B_{1}-2 \kappa_{10}^{2} T_{3} \tilde{\star} \tilde{Y}_{1} \tilde{\delta}^{6}(y, Y)\right]=0 .
$$

Equation (3.13) is the 6-dimensional dual of (3.9) and is thus automatically satisfied.

The D3-brane EOM (2.14) contributes no new constraints; the $M=\not$ component vanishes identically (see (C.23)) as in the background, while the $M=\not h$ component (shown below in (3.30)) contributes to the dynamical EOM only.

\subsubsection{Summary}

We have shown that the ansatz (3.1), (3.2), (3.3), (3.4) solves the constraints required to describe the motion of a D3-brane in a GKP background beyond the probe limit. To first order in the fluctuation of the brane position, the warp factor, metric and $\tilde{F}_{5}$ compensator, and Weyl factor are (repeating our earlier results)

$$
\begin{aligned}
e^{-4 A(x, y)} & =e^{-4 A^{(0)}(y)}+2 \kappa_{10}^{2} T_{3} \delta Y^{\eta_{h}} \partial_{\not h} \tilde{G}(y, Y), \\
B_{m}^{Y}(x, y) & =-2 \kappa_{10}^{2} T_{3} e^{-2 \Omega} \tilde{g}_{m n} \delta Y^{\not} \tilde{G}_{\not p}^{n}(y, Y), \\
e^{-2 \Omega(x)} & =\frac{1}{\tilde{V}} \int d^{6} y \sqrt{\tilde{g}} e^{-4 A^{(0)}(y)}=e^{-2 \Omega^{(0)}} .
\end{aligned}
$$

To our knowledge, this is the first ansatz in the literature that describes the backreaction of the D3-brane on the field strength and geometry and can be used to perform a consistent dimensional reduction.

\subsection{Kähler moduli in electric components}

Having found an ansatz for the SUGRA fields for a moving D3-brane that solves all constraints, we can determine the dynamical EOM and dimensionally reduce the quadratic action (2.4). However, the full structure of the effective action is apparent only when we include the complete moduli space. In this paper, we consider the universal volume modulus $c, 2$-form axions $b_{2}^{I}$ descending from $C_{4}$, and brane positions $Y^{\eta h}$. Each of the axions is 
associated with a harmonic 2-form $\omega_{2}^{I}$ on the unwarped CY manifold (where $I=1, \cdots h^{1,1}$ runs over a basis). In principle, the holomorphic moduli of the 4D SUGRA should include all the metric Kähler moduli as partners of the $b_{2}^{I}$; so far, the constraints have only been solved for the volume modulus. Some compactifications have additional axionic moduli that descend from $A_{2}$. Solutions to the constraints have been presented in [26], and they can be added to our analysis in a straightforward manner.

Here we present a brief description of the linearized fluctuations corresponding to all these moduli and the constraint equations they must solve; more details are presented in appendix C.1.

These moduli can all be described to linear order by a common metric

$$
d s^{2}=e^{2 \Omega} e^{2 A} \hat{\eta}_{\mu \nu} d x^{\mu} d x^{\nu}+2 e^{2 A} e^{2 \Omega} \partial_{\mu} B_{m} d x^{\mu} d y^{m}+e^{-2 A} \tilde{g}_{m n} d y^{m} d y^{n}
$$

and electric $C_{4}$ components $^{8}$

$$
C_{4}=e^{4 \Omega} e^{4 A} \hat{\epsilon}+e^{4 \Omega} e^{4 A_{\hat{\star}} \hat{d} B_{1}}+b_{2}^{I} \wedge \omega_{2}^{I} .
$$

The total compensator field is written as the sum from each moduli sector, so

$$
\hat{d} B_{1}(x, y) \equiv-\hat{d} c(x) \wedge \tilde{d} K(y)+e^{-4 \Omega_{\hat{\star}}} \hat{d} b_{2}^{I}(x) \wedge B_{1}^{b, I}(y)+\hat{d} B_{1}^{Y}(x, y) .
$$

Note that we have used a notational shorthand since $\hat{d}^{2} B_{1} \neq 0$ (that is, $\hat{d} B_{1}$ is not actually an exterior derivative in spacetime) except when the axions are on shell. It is worth noting that there is a gauge in which the volume modulus compensator field, $K(y)$, appears in the $(\mu \nu)$ component of the metric; however, this is not possible for the other moduli, since perturbations of $b_{2}^{I}$ and $Y^{\eta}$ directly source $T_{\mu m}$. The $C_{4}$ axions in the presence of background $G_{3}^{(0)}$ flux also source a compensator for the 2-form potential

$$
\delta A_{2}=-e^{-2 \Omega} \hat{\star} \hat{d} b_{2}^{I} \wedge \Lambda_{1}^{I} .
$$

The field strengths for our ansatz are

$$
\begin{aligned}
& \tilde{F}_{5}=e^{4 \Omega} \hat{\epsilon} \wedge \tilde{d} e^{4 A}+d\left(e^{4 \Omega} e^{4 A_{\hat{\star}} \hat{d} B_{1}}\right)+\hat{d} b_{2}^{I} \wedge \omega_{2}^{I} \\
& +\left[\tilde{\star} \tilde{d} e^{-4 A}-e^{2 \Omega} e^{-4 A_{\tilde{\star}}}\left(\hat{d} B_{1} \wedge \tilde{d} e^{4 A}\right)+\star d\left(e^{4 \Omega} e^{4 A_{\star} \hat{d} B_{1}}\right)+e^{-2 \Omega} \hat{\star} \hat{d} b_{2}^{I} \wedge e^{-4 A_{\tilde{\star}} \omega_{2}^{I}}\right], \\
& G_{3}=G_{3}^{(0)}-e^{-2 \Omega} \hat{d} \hat{\star} \hat{d} b_{2}^{I} \wedge \Lambda_{1}^{I}+e^{-2 \Omega} \hat{\star} \hat{d} b_{2}^{I} \wedge \tilde{d} \Lambda_{1}^{I} .
\end{aligned}
$$

As in the previous subsection, the terms in square brackets on the second line of (3.19) are the magnetic components dual to the electric components on the first line; the magnetic components subsume the Chern-Simons terms $\left(i g_{s} / 2\right)\left(\delta A_{2} \bar{G}_{3}^{(0)}-\delta \bar{A}_{2} G_{3}^{(0)}\right)$. This form for $\tilde{F}_{5}$ differs from the form presented in $[25,26]$ by terms proportional to the $4 \mathrm{D}$ dynamical EOM $e^{4 \Omega} e^{4 A} \hat{d} \hat{\star} \hat{d} B_{1}+e^{-4 A} \hat{\star} \hat{d} \hat{\star} \hat{d} \tilde{\star} B_{1}$. Therefore, both versions of the field strength represent the same on-shell degrees of freedom with slightly different $4 \mathrm{D}$ field definitions, and the constraints for the volume modulus and axions remain unchanged. We summarize them below.

\footnotetext{
${ }^{8}$ In principle, there can be an additional compensator term $-\hat{d} b_{2} K_{1}$ associated with each axion, but we show in the appendix that $K_{1}=0$.
} 
The constraints arising from the external components of the Einstein equation are, as above,

$$
\begin{aligned}
\tilde{\nabla}^{2} e^{-4 A(x, y)} & =-\frac{g_{s}}{2}\left|G_{3}^{(0)}\right|^{\tilde{2}}-2 \kappa_{10}^{2} T_{3} \tilde{\delta}^{6}(y, Y)-\cdots, \\
\tilde{\nabla}^{\tilde{n}} B_{n} & =e^{-2 \Omega} \delta e^{-4 A}-e^{-4 A} \delta e^{-2 \Omega}
\end{aligned}
$$

Since the axions do not appear as sources for the warp factor in (3.21), the warp and Weyl factors are independent of these degrees of freedom. However, the volume modulus appears as a spacetime-dependent shift of the warp factor [25]

$$
e^{-4 A(x, y)}=e^{-4 A^{(0)}(y)}+c(x)+2 \kappa_{10}^{2} T_{3} \delta Y^{\eta h}(x) \partial_{\not h} \tilde{G}(y, Y) .
$$

The Weyl factor is $e^{-2 \Omega}=e^{-2 \Omega^{(0)}}+c(x)$ including the volume modulus. In addition to (3.6), (3.22) gives a Poisson equation for the volume modulus compensator

$$
\tilde{\nabla}^{2} K(y)=e^{-4 A^{(0)}(y)}-e^{-2 \Omega^{(0)}}
$$

and $\tilde{\nabla}^{\tilde{n}} B_{n}^{b, I}=0$.

The mixed component of $E_{M N}$ gives a nontrivial constraint for all of the degrees of freedom, namely

$$
\begin{aligned}
& -\frac{1}{2} e^{4 A} \partial_{\mu} \partial_{m} e^{-4 A}+\frac{1}{2} e^{2 \Omega} e^{4 A} \partial_{\mu} \tilde{\nabla}^{\tilde{n}}\left(\tilde{d} B_{1}\right)_{m n}+\kappa_{10}^{2} T_{3} e^{4 A} \tilde{g}_{m \eta} \partial_{\mu} Y^{n} \tilde{\delta}^{6}(y, Y) \\
& -2 e^{-2 \Omega} e^{4 A}\left(\hat{\star} \hat{d} b_{2}^{I}\right)_{\mu}\left[e^{-4 A}\left(\omega_{2}^{I}\right)_{m n} \partial^{\tilde{n}} A-\frac{i g_{s}}{8}\left(\tilde{\star}\left(\tilde{d} \Lambda_{1} \wedge \bar{G}_{3}^{(0)}\right)_{m}-\text { c.c. }\right)\right]=0,
\end{aligned}
$$

as well as a copy of equation (3.21) multiplied by $\partial_{\mu} B_{m}$. We have already seen how this equation is satisfied for fluctuations in D3-brane position; for the volume modulus, we note that $\partial_{\mu} \partial_{m} e^{-4 A}=0$ and that its compensator is exact, so the constraint is automatically satisfied. For the 2-form axions, (3.25) becomes

$$
\tilde{\nabla}^{2} B_{m}^{b, I}=4 e^{-4 A}\left(\omega_{2}^{I}\right)_{m n} \partial^{\tilde{n}} A-\frac{i g_{s}}{2} \tilde{\star}\left(\tilde{d} \Lambda_{1}^{I} \wedge \bar{G}_{3}^{(0)}-\text { c.c. }\right),
$$

which we take to be the defining Poisson equation for the axion compensators, as in [26].

The constraints coming from the 5 -form EOM (C.20) again include a copy of (3.21), as well as $\tilde{\star}$ of $(3.25)$. For the axions, we can further rewrite this constraint as

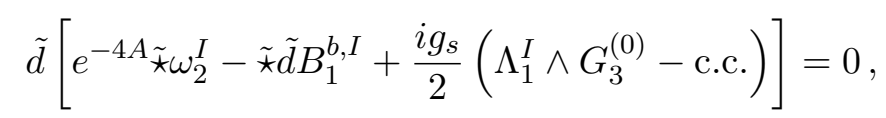

which implies that

$$
e^{-4 A_{\tilde{\star}} \omega_{2}^{I}}+\frac{i g_{s}}{2}\left(\Lambda_{1}^{I} \wedge G_{3}^{(0)}-\text { c.c. }\right)-\tilde{\star} \tilde{d} B_{1}^{b, I}=\gamma_{4}^{I}+\tilde{d} K_{3}^{I},
$$

where $\gamma_{4}^{I}$ is harmonic and $K_{3}$ is given by a Poisson-like equation (whose precise form will be unimportant to us).

Finally, the constraint from the 3 -form EOM (2.13) as given in (C.21) receives no contribution from the volume or D3 position moduli. For the axions, it is

$$
\tilde{d} \tilde{\star} \tilde{d} \Lambda_{1}+i \omega_{2}^{I} \wedge G_{3}^{(0)}=0 .
$$

The contribution $\Lambda_{1}^{I}$ for each axion takes the same form as in [26]. 


\subsubsection{Summary}

We have shown that a generic "electric" ansatz, given by (3.15), (3.16), (3.17), (3.19), can be used to describe the volume modulus, $C_{4}$ axions, and D3-brane position. The warp factor, Weyl factor, and compensators for each modulus can be determined by (3.23), (3.24), (3.26) and (3.29) - along with corresponding expressions from section 3.1 - and are shown to satisfy all of the 10D constraint equations. It is important to note that the constraints require fluctuations in multiple $10 \mathrm{D}$ fields for each of the moduli separately.

\subsection{D effective action in electric formalism}

As discussed in section 2.2, the quadratic effective action is obtained by multiplying the fluctuations of the 10D fields with the first-order parts of the 10D dynamical EOM; in this case, there is an additional contribution to the quadratic action described below that takes a topological form and does not appear in the EOM. Integration over the compact manifold projects onto the massless sector.

\subsubsection{Contributions to effective action}

As described above, the quadratic action is given by

$$
\begin{aligned}
S= & \frac{1}{4 \kappa_{10}^{2}} \int d^{10} x \sqrt{-g} \delta g^{M N} \delta E_{M N}+\frac{1}{4 \kappa_{10}^{2}} \int\left(\delta C_{4} \wedge \delta E_{6}+\frac{g_{s}}{2} \delta A_{2} \wedge \delta \bar{E}_{8}+\frac{g_{s}}{2} \delta \bar{A}_{2} \wedge \delta E_{8}\right) \\
& +\frac{T_{3}}{2} \int d^{10} x \sqrt{-g} \int d^{4} \xi \sqrt{-\gamma} \delta X^{M} \delta E_{M I},
\end{aligned}
$$

where $\delta g_{M N}, \delta C_{4}, \delta A_{2}$ are the first-order parts of (3.15), (3.16), (3.18). However, as noted in section 2.2, there can generally be "topological" terms in the quadratic action, such as the instanton density of 4D Yang-Mills theory, that do not appear in the linearized EOM. We identify a contribution of this type below.

We begin with the contribution from the D3-brane sector. The dynamical part of the D3-brane EOM is

$$
\begin{aligned}
\delta E_{\not h} & =e^{-4 A} e^{-4 \Omega}\left(e^{2 \Omega} \tilde{g}_{\not h \eta h} \hat{\partial}^{2} Y^{\not h}+e^{4 A} e^{4 \Omega} \hat{\partial}^{2} B_{\not h}-e^{4 A} e^{4 \Omega} \hat{\partial}^{2} B_{\not h}\right) \delta^{10}(x, X) \\
& =e^{-4 A} e^{-2 \Omega} \tilde{g}_{\not h \eta} \hat{\partial}^{2} Y^{h} \delta^{10}(x, X) .
\end{aligned}
$$

Note that the contribution to the EOM from the WZ action has cancelled with a term proportional to $g_{\mu m}$ in the DBI action in the first line of (3.30). We obtain the contribution

$$
S_{\text {eff }}^{D 3}=\frac{T_{3}}{2} \int d^{4} x \int d^{6} y \sqrt{\tilde{g}} e^{2 \Omega} \tilde{g}_{\eta \not h} \delta Y^{\not h} \hat{\partial}^{2} Y^{\not h} \tilde{\delta}^{6}(y, Y) .
$$

There is no "self-energy" problem; the singular fields sourced by the D3-brane cancel out of the effective action. Previous attempts at constructing the effective action for D3-branes (such as $[15,27]$ ) have only included this contribution from the DBI action on a fixed warped background. However, as we have emphasized, there are, in principle, additional contributions to the effective action arising from the presence of moduli dependence in the metric and flux sectors. 
For example, the dynamical Einstein equations, given in (C.17), (C.18), (C.19), are

$$
\begin{aligned}
\delta E_{\mu \nu} & =-2 e^{4 A} e^{2 \Omega} \hat{\eta}_{\mu \nu}\left(\partial^{\tilde{\ell}} A\right) \hat{\partial}^{2} B_{\ell}, \\
\delta E_{\mu m} & =0, \\
\delta E_{m n} & =\hat{\partial}^{2}\left[\tilde{\nabla}_{(m} B_{n)}-\tilde{g}_{m n} \tilde{\nabla}^{\tilde{\ell}} B_{\ell}\right]+e^{-4 A} e^{-2 \Omega} \tilde{g}_{m n} \hat{\partial}^{2}(3 \delta \Omega-2 \delta A) .
\end{aligned}
$$

Inserting these into the expression for the gravity contribution to the effective action, we obtain

$$
\begin{aligned}
S_{\mathrm{eff}}^{R}= & \frac{1}{4 \kappa_{10}^{2}} \int d^{4} x \int d^{6} y \sqrt{\tilde{g}} e^{4 \Omega}\left[16(\delta A+\delta \Omega)\left(\partial^{\tilde{\ell}} A\right) \hat{\partial}^{2} B_{\ell}-10 \delta A \hat{\partial}^{2} \tilde{\nabla}^{\tilde{\ell}} B_{\ell}\right. \\
& \left.+12 e^{-4 A} e^{-2 \Omega} \delta A \hat{\partial}^{2}(3 \delta \Omega-2 \delta A)\right] .
\end{aligned}
$$

Notice that (3.35) implies that there are contributions to the effective action for D3-brane fluctuations from both the compensators and the fluctuations in the warp and Weyl factors. These include complicated mixings with the volume modulus and $C_{4}$ axions and depend on the details of the solutions for the compensators from (3.24) and (3.26).

Next, we need to include the contribution to the effective action from the flux sectors. The relevant ${ }^{9}$ dynamic contributions to the 5 -form EOM are

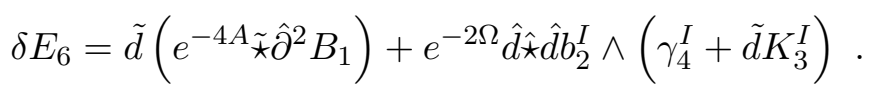

The 5 -form contribution to the effective action is then

$$
\begin{aligned}
S_{\text {eff }}^{5}= & \frac{1}{4 \kappa_{10}^{2}} \int d^{4} x \int d^{6} y \sqrt{\tilde{g}} e^{4 \Omega}\left[4(\delta A+\delta \Omega)\left(\hat{\partial}^{2} \tilde{\nabla}^{\tilde{\ell}} B_{\ell}-4 \partial^{\tilde{\ell}} A \hat{\partial}^{2} B_{\ell}\right)\right] \\
& -\frac{1}{4 \kappa_{10}^{2}} \int e^{-2 \Omega} \hat{d} b_{2}^{I} \wedge \hat{\star} \hat{d} b_{2}^{J} \int \omega_{2}^{I} \wedge \gamma_{4}^{J} .
\end{aligned}
$$

We expand $\gamma_{4}^{J}=\left(C^{-1}\right)^{J K} \tilde{\star} \omega_{2}^{K}$, so $\int \omega_{2}^{I} \gamma_{4}^{J}=3 \tilde{V}\left(C^{-1}\right)^{I J}$ using the normalization of our basis forms. Following [26],

$$
\left(C^{-1}\right)^{I J}=\frac{1}{3 \tilde{V}}\left\{\int e^{-4 A} \omega_{2}^{I} \wedge \tilde{\star} \omega_{2}^{J}+\frac{i g_{s}}{2} \int \omega_{2}^{I} \wedge\left(\Lambda_{1}^{J} \wedge \bar{G}_{3}^{(0)}-\bar{\Lambda}_{1}^{J} \wedge G_{3}^{(0)}\right)\right\},
$$

which is the identity when the CY metric is formal, meaning that the wedge product of harmonic forms is always harmonic. Meanwhile, the 3-form sector does not contribute to the quadratic action ${ }^{10}$ because none of the components of $\delta E_{8}$ have the correct legs to wedge nontrivially with $\delta A_{2}$.

Finally, there is an additional contribution to the effective action that is not captured by equation (2.4). Specifically, the WZ action (2.9) contains a term that is quadratic in

\footnotetext{
${ }^{9}$ There is an additional term of the form $e^{-4 A} \tilde{\star} \hat{d} \hat{\partial}^{2} B_{1}$, but it contributes only to higher-derivative terms. We expect these to be modified by other corrections, such as threshold corrections. They are also ambiguous because they change under field redefinitions of the form $u \rightarrow u+f(u) \hat{\partial}^{2} u$. For both these reasons, we do not consider them.

${ }^{10}$ Except at higher derivatives, which we ignore for the reasons stated in footnote 9.
} 
field fluctautions but does not give a contribution to the linearized EOM: ${ }^{11}$

$$
S_{\text {eff }}^{a x-D 3}=\frac{T_{3}}{2} \int_{D 3} b_{2}^{I} \wedge \hat{d} Y^{\not h} \wedge \hat{d} Y^{\not h} \omega_{\eta \not h}^{I} .
$$

This is analogous to the axion-photon coupling $\theta F^{2}$, which contributes to the EOM only at quadratic order in the fields but can nonetheless contribute to the action at quadratic order in fluctuations if $\theta$ has a background value. In infinite Minkowski space, a constant background $b_{2}$ is gauge trivial, but there are nontrivial Wilson lines if the spatial dimensions are compactified on a large torus, for example, so we must include this term. The terms in the action with background $b_{2}$ are topological in the sense that they are total derivatives, also like the axion-photon coupling.

Adding all the contributions yields a series of cancellations. Specifically, all terms proportional to $\partial^{\tilde{\ell}} A \hat{\partial}^{2} B_{\ell}$ cancel. Then the constraint equation for the compensator (3.22) allows us to simplify the remaining terms involving $\delta A, \delta \Omega$ to the form

$$
\begin{aligned}
S_{\mathrm{eff}}^{A-\Omega} & =\frac{8}{4 \kappa_{10}^{2}} \int d^{4} x \int d^{6} y \sqrt{\tilde{g}} e^{-4 A} e^{2 \Omega}(\delta A-\delta \Omega) \hat{\partial}^{2} \delta \Omega \\
& =\frac{1}{4 \kappa_{10}^{2}} \int d^{4} x \int d^{6} y \sqrt{\tilde{g}}\left[e^{4 \Omega}\left(c+2 \kappa_{10}^{2} T_{3} \delta Y^{\eta h} \partial_{\eta p} \tilde{G}(y ; Y)\right) \hat{\partial}^{2} c+2 e^{6 \Omega} e^{-4 A} c \hat{\partial}^{2} c\right] .
\end{aligned}
$$

Since $\partial_{\eta_{n}} \tilde{G}(y ; Y)=-\tilde{\nabla}_{n} \tilde{G}_{m p}^{n}(y ; Y)$, the brane-volume cross-term integrates to zero. With the definition of the Weyl factor, the remaining terms combine, yielding

$$
\begin{aligned}
& S_{\mathrm{eff}}=S_{\mathrm{eff}}^{D 3}+S_{\text {eff }}^{R}+S_{\text {eff }}^{5}+S_{\text {eff }}^{a x-D 3} \\
& =-\frac{3 \tilde{V}}{4 \kappa_{10}^{2}} \int d^{4} x e^{4 \Omega} \partial^{\hat{\mu}} c(x) \partial_{\mu} c(x)-\frac{3 \tilde{V}}{4 \kappa_{10}^{2}} \int e^{-2 \Omega}\left(C^{-1}\right)^{I J} \hat{d} b_{2}^{I} \wedge \hat{\star} \hat{d} b_{2}^{J}
\end{aligned}
$$

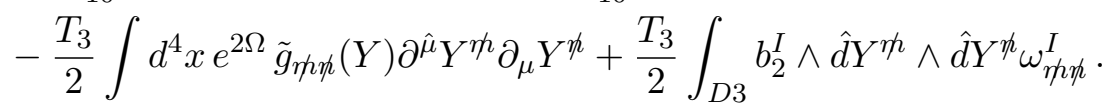

This is the key result of this section: all of the complicated structures from the ansatz, including compensators and Green's functions, end up cancelling non-trivially in the final effective action. This remarkable set of cancellations illustrates the necessity for a consistent solution of the 10D constraint equations; the constraints, and their solutions, are essential for simplifying the effective action. Note that the only contribution to the effective action by the warp factor is through the axion field space metric (3.38), as was determined in [26].

\subsubsection{Scalar axions and kinetic action}

Since the $4 \mathrm{D}$ effective theory is a supergravity, ultimately the quantity of interest is the Kähler potential, so we should write the kinetic action in terms of holomorphic scalar coordinates. Here, we dualize the 2-form axions into scalars and find that using complex coordinates $y^{m}=\left(z^{i}, \bar{z}^{\bar{\imath}}\right)$ on the $\mathrm{CY}$ provides some simpification.

The CY coordinates appear explicitly in the action (3.41) through the brane positions

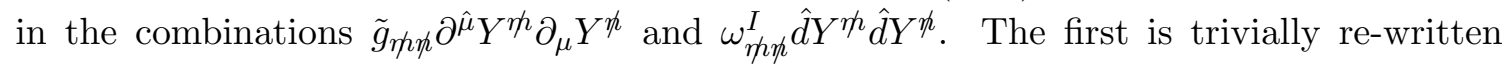

\footnotetext{
${ }^{11}$ The extra factor of $1 / 2$ in (3.39) compared to the WZ form (2.9) follows from careful comparison of combinatorial factors in a general 4 -form versus a 2 -form wedged with two 1-forms.
} 
in complex coordinates as $2 \tilde{g}_{i \bar{\jmath}} \partial^{\hat{\mu}} Z^{i} \partial_{\mu} \bar{Z}^{\bar{\jmath}}$ (for legibility, we drop the slash on quantities evaluated at the brane position in complex coordinates). However, we can re-organize the second further; by the $\partial \bar{\partial}$ lemma, the harmonic 2-forms are locally $\omega_{2}^{I}=i \partial \bar{\partial} k^{I}(z, \bar{z})$ in terms of potentials $k^{I}$ (when $\omega_{2}$ is the complex structure form $\tilde{J}_{2}, k$ is the Kähler potential of the CY), and

$$
\omega_{\eta \not h}^{I} \hat{d} Y^{\eta h} \hat{d} Y^{\natural \hbar}=i \partial_{i} k_{\bar{\jmath}}^{I} \hat{d} Z^{i} \hat{d} \bar{Z}^{\bar{\jmath}}-i \bar{\partial}_{\bar{\imath}} k_{j}^{I} \hat{d} \bar{Z}^{\bar{\imath}} \hat{d} Z^{j}, \quad k_{i}^{I}=\partial_{i} k^{I}, \quad k_{\bar{\imath}}^{I}=\bar{\partial}_{\bar{\imath}} k^{I} .
$$

Furthermore, since $k$ is evaluated at the brane position, we have $\hat{d} k_{\bar{\jmath}}^{I}=\partial_{i} k_{\bar{\jmath}} \hat{d} Z^{i}+\partial_{\bar{\imath}} k_{\bar{\jmath}}^{I} \hat{d} Z^{\bar{\jmath}}$; since partial derivatives commute, $\hat{d} k_{\bar{\jmath}}^{I} \hat{d} \bar{Z}^{\bar{\jmath}}=\partial_{i} k_{\bar{\jmath}}^{I} \hat{d} Z^{i} \hat{d} \bar{Z}^{\bar{j}}$.

Now the kinetic action for the axions can be written as

$$
\begin{aligned}
S_{\text {axion }} & =-\frac{3 \tilde{V}}{4 \kappa_{10}^{2}} \int e^{-2 \Omega}\left(C^{-1}\right)^{I J} \hat{d} b_{2}^{I} \wedge \hat{\star} \hat{d} b_{2}^{J}+i \frac{T_{3}}{2} \int b_{2}^{I} \wedge\left(\hat{d} k_{\bar{\imath}}^{I} \wedge \hat{d} \bar{Z}^{\bar{\imath}}-\hat{d} k_{i}^{I} \wedge \hat{d} Z^{i}\right) \\
& =-\frac{3 \tilde{V}}{4 \kappa_{10}^{2}} \int\left[e^{-2 \Omega}\left(C^{-1}\right)^{I J} \hat{d} b_{2}^{I} \wedge \hat{\star} \hat{d} b_{2}^{J}-i \gamma \hat{d} b_{2}^{I} \wedge \kappa_{1}^{I},\right]
\end{aligned}
$$

where we have defined $\kappa_{1}^{I} \equiv k_{j}^{I} \hat{d} Z^{j}-k_{\bar{\jmath}}^{I} \hat{d} Z^{\bar{j}}$ and $\gamma=2 \kappa_{10}^{2} T_{3} / 3 \tilde{V}$. To define the scalar axion, we re-write the action (3.43) in terms of the field strength $h_{3}^{I} \equiv \hat{d} b_{2}^{I}$ and enforce the Bianchi identity $\hat{d} h_{3}^{I}=0$ by introducing a Lagrange multiplier $b_{0}^{I}$, so

$$
S_{\text {axion }}=-\frac{3 \tilde{V}}{4 \kappa_{10}^{2}} \int\left(e^{-2 \Omega}\left(C^{-1}\right)^{I J} h_{3}^{I} \wedge \hat{\star} h_{3}^{J}-i \gamma h_{3}^{I} \wedge \kappa_{1}^{I}-2 b_{0}^{I} \hat{d} h_{3}^{I}\right) .
$$

The classical EOM implies

$$
h_{3}^{I}=e^{2 \Omega} C^{I J} \hat{\star}\left(\hat{d} b_{0}^{J}+i \frac{\gamma}{2} \kappa_{1}^{J}\right) .
$$

Substituting this into (3.44) and simplifying gives the action in terms of the scalar axions as

$$
S_{\text {axion }}=-\frac{3 \tilde{V}}{4 \kappa_{10}^{2}} \int e^{2 \Omega} C^{I J}\left(\hat{d} b_{0}^{I}+i \frac{\gamma}{2} \kappa_{1}^{I}\right) \wedge \hat{\star}\left(\hat{d} b_{0}^{J}+i \frac{\gamma}{2} \kappa_{1}^{J}\right) .
$$

With the brane coordinates written in terms of complex variables and the axions dualized to the conventional scalars, the effective action (3.41) becomes

$$
\begin{aligned}
S_{\text {eff }}= & -\frac{3 \tilde{V}}{4 \kappa_{10}^{2}} \int d^{4} x\left[e^{4 \Omega} \partial^{\hat{\mu}} c \partial_{\mu} c+2 \gamma e^{2 \Omega} \tilde{g}_{i \bar{\jmath}}(Z, \bar{Z}) \partial^{\hat{\mu}} Z^{i} \partial_{\mu} \bar{Z}^{\bar{\jmath}}+e^{2 \Omega} C^{I J}\right. \\
& \left.\times\left(\partial^{\hat{\mu}} b^{I}+i \frac{\gamma}{2} k_{i}^{I} \partial^{\hat{\mu}} Z^{i}-i \frac{\gamma}{2} k_{\bar{\imath}}^{I} \partial^{\hat{\mu}} \bar{Z}^{\bar{\imath}}\right)\left(\partial_{\mu} b^{J}+i \frac{\gamma}{2} k_{j}^{J} \partial_{\mu} Z^{j}-i \frac{\gamma}{2} k_{\bar{\jmath}}^{J} \partial_{\mu} \bar{Z}^{\bar{\jmath}}\right)\right] .
\end{aligned}
$$

This effective action for the volume modulus, $C_{4}$ axions, and D3-brane positions is the primary result of this paper. Compared to the action found in [1], which does not account for the effects of the warp factor or flux in kinetic terms, our result is similar, but we find that a nontrivial warp factor and flux appear through the Weyl factor (which corresponds to shifting the expectation value of the volume modulus) and the metric $C^{I J}$, as in $[25,26]$. In fact, our result matches that derived in [18] using methods from 4D conformal SUGRA 
and corrected by flux dependence on metric moduli [19]; the contribution from the flux background to $C^{I J}$ already appeared in direct dimensional reduction in [26].

In the case that the CY metric $\tilde{g}_{m n}$ is formal or that we restrict to the universal axion ( $\omega_{2}=\tilde{J}_{2}$, the almost complex structure), $C^{I J}=e^{2 \Omega} \delta^{I J}$. With a single axion $b$, then the effective action (3.47) follows from the Kähler potential

$$
\mathcal{K}=-3 \ln [-i(\rho-\bar{\rho})-\gamma k(Z, \bar{Z})]
$$

and holomorphic coordinate

$$
\rho=b_{0}+i\left(e^{-2 \Omega}+\frac{\gamma}{2} k(Z, \bar{Z})\right)
$$

as we show in appendix D. This takes the form proposed in $[7,15]$ for D3-branes in warped compactifications when restricted to a single Kähler modulus.

\subsubsection{Summary}

Starting with the ansatz of the previous subsection for the 10D SUGRA fields, we have performed a consistent dimensional reduction beyond the probe limit of the effective action of a mobile D3-brane, the volume modulus, and 4-form axions in a warped GKP background. A number of critical cancellations occur because the 10D fields satisfy their constraint equations.

The effective action is

$$
\begin{aligned}
S_{\text {eff }}= & -\frac{3 \tilde{V}}{4 \kappa_{10}^{2}} \int d^{4} x\left[e^{4 \Omega} \partial^{\hat{\mu}} c \partial_{\mu} c+\gamma e^{2 \Omega} \tilde{g}_{i \bar{\jmath}}(Z, \bar{Z}) \partial^{\hat{\mu}} Z^{i} \partial_{\mu} \bar{Z}^{\bar{\jmath}}+e^{2 \Omega} C^{I J}\right. \\
& \left.\times\left(\partial^{\hat{\mu}} b^{I}+i \frac{\gamma}{2} k_{i}^{I} \partial^{\hat{\mu}} Z^{i}-i \frac{\gamma}{2} k_{\bar{\imath}}^{I} \partial^{\hat{\mu}} \bar{Z}^{\bar{\imath}}\right)\left(\partial_{\mu} b^{J}+i \frac{\gamma}{2} k_{j}^{J} \partial_{\mu} Z^{j}-i \frac{\gamma}{2} k_{\bar{\jmath}}^{J} \partial_{\mu} \bar{Z}^{\bar{\jmath}}\right)\right],
\end{aligned}
$$

where the flux and warp factor appear through the metric

$$
\left(C^{-1}\right)^{I J}=\frac{1}{3 \tilde{V}}\left\{\int e^{-4 A} \omega_{2}^{I} \wedge \tilde{\star} \omega_{2}^{J}+\frac{i g_{s}}{2} \int \omega_{2}^{I} \wedge\left(\Lambda_{1}^{J} \wedge \bar{G}_{3}^{(0)}-\bar{\Lambda}_{1}^{J} \wedge G_{3}^{(0)}\right)\right\} .
$$

There is no known explicit form for the corresponding Kähler potential, though it reduces to the DeWolfe-Giddings [7, 15] form when the CY has only a single Kähler modulus.

\section{Magnetic D3-brane couplings}

We can also carry out the dimensional reduction in the version of type IIB SUGRA in which we keep the mostly internal components of $\tilde{F}_{5}$. In this version of the theory, a D3-brane does not couple to $C_{4}$ in the action, ${ }^{12}$ but rather through a nontrivial Bianchi identity. To describe the SUGRA in this way will require a new, but equivalent, expression for the field strength ansatz which differs from (3.4) by terms that are proportional to equations of motion. Since it is identical on shell, this ansatz describes the same 4D degrees of freedom as (3.4); off shell, the $4 \mathrm{D}$ effective action will differ only in higher-derivative terms, which

\footnotetext{
${ }^{12}$ Except at higher order in spacetime derivatives than we consider.
} 
are ambiguous as they can be changed by a $4 \mathrm{D}$ field redefinition. We start this section by demonstrating that the new ansatz satisfies the same constraints as required in the electric description of the D3-brane.

At the same time, we will notice that the fluctuation in $\tilde{F}_{5}$ decomposes into an exact term (the contribution of a globally-defined 4-form potential) and a delta-function supported term with an explicit dependence on the brane position. This motivates us to divide $\tilde{F}_{5}$ in the presence of magnetic charges into an exact piece and terms that depend explicitly on brane position; the field redefinition yields a 4 -form potential without a Dirac string singularity, much as a field redefinition can be used to create a 4 -form potential that is invariant under 2-form gauge transformations needed to describe a background 3-form field strength. We discuss this field redefinition in section 4.2.

A puzzle that arises when treating the D3-brane as a magnetic charge is how the no-force condition in our background arises, since there is no WZ coupling between the brane and $C_{4}$. As it turns out, the field redefinition described above solves this puzzle, since the explicit dependence of $\tilde{F}_{5}$ on the brane position modifies the D3-brane EOM. In section 4.2.3, we find the modified equation of motion for the brane and demonstrate that a static D3-brane feels no force in backgrounds that are mutually BPS with the brane (including GKP backgrounds).

Finally, after reviewing the results of $[25,26]$ in a "magnetic" description, we present a unified ansatz for the volume modulus, $C_{4}$ axions, and brane position at linear order. Using this ansatz, we solve the constraints and integrate the quadratic action over the internal manifold to find the $4 \mathrm{D}$ effective action for all moduli.

\subsection{D3-brane fluctuations in magnetic formalism}

Clearly, to represent the same $4 \mathrm{D}$ degree of freedom, the self-dual $\tilde{F}_{5}$ must be the same on shell whether we choose to describe IIB SUGRA using the electric or magnetic components. However, the magnetic components of $\tilde{F}_{5}$ in the ansatz (3.4) (which we used for the electrically-coupled D3-brane) are not easily described in terms of a 4-form potential with the magnetic set of components. Fortunately, it is possible to describe the same on-shell solution of the $10 \mathrm{D}$ theory by adding terms proportional to the $4 \mathrm{D}$ dynamical EOM to $\tilde{F}_{5}$; some of these combine with other magnetic components to take the form $d C_{4}$, as we will see below. Specifically, we take

$$
\begin{aligned}
\tilde{F}_{5}^{\text {mag }} & =\tilde{F}_{5}^{\text {elec }}-e^{4 A} e^{4 \Omega} \hat{d} \hat{\star} \hat{d} B_{1}^{Y}-e^{-4 A} \hat{\star} \hat{d} \hat{\star} \hat{d} \tilde{\star} B_{1}^{Y} \\
& =\tilde{\star} \tilde{d} e^{-4 A}-e^{2 \Omega} \hat{d}\left(\tilde{\star} \tilde{d} B_{1}^{Y}\right)+\left[e^{4 \Omega} \hat{\epsilon} \wedge \tilde{d} e^{4 A}-e^{4 \Omega} \hat{\star} \hat{d} \tilde{d}\left(e^{4 A} B_{1}^{Y}\right)\right],
\end{aligned}
$$

where $\tilde{F}_{5}^{\text {elec }}$ is given by (3.4). In the second line, the first two terms are the magnetic components of $\tilde{F}_{5}^{\text {mag }}$, while the terms in brackets are the electric components. Throughout the remainder of this section, we consider only $\tilde{F}_{5}^{\text {mag }}$, and therefore we suppress the superscript. Our ansatz for the metric remains the same.

Since it differs from the ansatz in the electric formalism only by terms that are second order in spacetime derivatives, this modified ansatz for $\tilde{F}_{5}$ leaves the constraint equations unchanged, so it is still a valid ansatz for dimensional reduction. The additional terms 
in (4.1) lead to higher-derivative terms in the dynamical EOM, which in fact vanish on shell and do not affect our analysis. We verify that the constraints are unchanged by explicit calculation in appendix C.2.

We can now address our claim that the magnetic components of this ansatz are simply written in terms of a 4 -form potential. The key is the constraint (3.13), which can be written as

$$
\tilde{\star} \tilde{d} \delta e^{-4 A}+e^{2 \Omega} \tilde{d} \tilde{\star} \tilde{d} B_{1}-2 \kappa_{10}^{2} T_{3} \tilde{\star} \tilde{Y}_{1} \tilde{\delta}^{6}(y, Y)=0 .
$$

As a result, the fluctuation in the magnetic components is

$$
\begin{aligned}
\delta \tilde{F}_{5} & =\tilde{\star} \tilde{d} \delta e^{-4 A}-e^{2 \Omega_{\tilde{\star}} \tilde{d} \hat{d} B_{1}^{Y}} \\
& =-d\left(e^{2 \Omega_{\tilde{\star}} \tilde{d} B_{1}^{Y}}\right)+2 \kappa_{10}^{2} T_{3} \tilde{\star} \tilde{Y}_{1} \tilde{\delta}^{6}(y, Y) .
\end{aligned}
$$

In other words, $\delta \tilde{F}_{5}=d \delta C_{4}^{\prime}+S_{5}$, where $C_{4}^{\prime}$ is a globally-defined potential (ie, has no Dirac string singularity and can therefore be defined with a single gauge patch) and $S_{5}$ is the explicit dependence on the brane position required to solve the Bianchi identity. This is a (non-local) field redefinition of the potential which apparently gives the 5-form an explicit dependence on the brane position. We will explore this field redefinition in more detail in the following subsection, including an analogy to the well-known Chern-Simons terms involving $A_{2}$ and $G_{3}$ in $\tilde{F}_{5}$.

\subsubsection{Summary}

The ansatz for 10D fields is somewhat modified in order to write the magnetic components of $\tilde{F}_{5}$ in terms of a 4 -form potential. The metric and 5 -form are given by

$$
\begin{aligned}
d s^{2} & =e^{2 \Omega} e^{2 A} \hat{\eta}_{\mu \nu} d x^{\mu} d x^{\nu}+2 e^{2 \Omega} e^{2 A} \partial_{\mu} B_{m}^{Y} d x^{\mu} d y^{m}+e^{-2 A} \tilde{g}_{m n} d y^{m} d y^{n}, \\
\tilde{F}_{5} & =\tilde{\star} \tilde{d} e^{-4 A}-e^{2 \Omega} \hat{d}\left(\tilde{\star} \tilde{d} B_{1}^{Y}\right)+\left[e^{4 \Omega} \hat{\epsilon} \wedge \tilde{d} e^{4 A}-e^{4 \Omega} \hat{\star} \hat{d} \tilde{d}\left(e^{4 A} B_{1}^{Y}\right)\right],
\end{aligned}
$$

where the magnetic components are the first two terms. We have found that the magnetic components of the field strength can be written as $\tilde{F}_{5}=S_{5}+d C_{4}^{\prime}$, where $S_{5}$ contains explicit dependence on the brane position and is described by a potential with a Dirac string singularity. The redefined potential $C_{4}^{\prime}$ is globally defined and has fluctuation $\delta C_{4}^{\prime}=$ $-e^{2 \Omega} \tilde{\star} \tilde{d} B_{1}^{Y}$.

This ansatz satisfies the same constraints as the ansatz we proposed in the electric formalism. Therefore, we still have $e^{2 \Omega}=e^{2 \Omega^{(0)}}$,

$$
\begin{aligned}
& e^{-4 A(x, y)}=e^{-4 A^{(0)}(y)}+2 \kappa_{10}^{2} T_{3} \delta Y^{\not h} \partial_{\not h} \tilde{G}(y, Y), \text { and } \\
& B_{m}^{Y}(x, y)=-2 \kappa_{10}^{2} T_{3} e^{-2 \Omega} \tilde{g}_{m n} \delta Y^{\not p} \tilde{G}_{\not p}^{n}(y, Y) .
\end{aligned}
$$

\subsection{Non-trivial Bianchi identities, field redefintions, and EOM}

We recall from equation (2.12) that the Bianchi identity for the magnetic components of $\tilde{F}_{5}$ is

$$
d \tilde{F}_{5}=\frac{i g_{s}}{2} G_{3} \wedge \bar{G}_{3}-2 \kappa_{10}^{2} T_{3} \sum_{D 3 / O 3} \int d^{4} \xi \sqrt{-\gamma} \star \epsilon_{\|} \delta^{10}(x, X(\xi))
$$


which has both distributed $\left(G_{3}\right)$ and local sources. The meaning of the distributed sources is well-understood - the gauge-invariant field strength contains both an exact term and Chern-Simons terms involving both the potential and field strength for another SUGRA degree of freedom. As a result, the 4 -form potential $C_{4}$ (even at first order in perturbations) has a nontrivial gauge patching in a background $G_{3} ;[21,25,26]$ demonstrated that this gauge transformation can be removed from perturbations of $C_{4}$ by a simple field redefinition, so the perturbation in $\tilde{F}_{5}$ decomposes into an exact term and a (somewhat altered) Chern-Simons term.

Similarly, the presence of a local magnetic source implies that $C_{4}$ must be defined on at least two patches glued together with a nontrivial gauge transformation (to remove the Dirac-string-like singularity). We show here that, as in the case of distributed sources, there is a field redefinition of the potential that allows the perturbation in the field strength to be written as $d C_{4}^{\prime}$ plus an analog of Chern-Simons terms with delta-function support. Our approach is to make a formal expansion of the source terms around an arbitrary fixed point; the nontrivial gauge patching can then be relegated to a background potential that creates the zeroth order term in the expansion, while the spacetime-dependent terms in the field strength are separated into an explicit dependence on the brane position and the exterior derivative of an exact potential. This explicit dependence of the field strength on the D3-brane position in turn modifies the EOM for the brane's motion. These techniques are similar to Dirac's original proposal for magnetic monopoles in 4D Maxwell theory [28]; the relationship of our work to Dirac's and the extension of Dirac's formalism to general branes is the subject of an upcoming companion paper by two of us [29].

The key point in both cases is two-fold: the original $C_{4}$ is not suitable for dimensional reduction because it is not globally defined (and cannot be integrated over the CY in the usual way, for example) and is not an entirely independent degree of freedom because its nontrivial gauge patching depends on the values of other fields. The field redefinitions we discuss below resolve both of these difficulties.

We begin with a brief review of the field redefinition in the case of a background $G_{3}$, largely following [26]. We then demonstrate how to re-write $\tilde{F}_{5}$ with explicit dependence on the brane position in a generic background, working in static gauge. The cases of distributed and local magnetic sources are independent, so we discuss them separately. We close with a discussion of the modified D3-brane EOM in section 4.2.3.

\subsubsection{Field redefinition in background 3-Form}

Ignoring local sources, the 5 -form Bianchi identity can be written as $d \tilde{F}_{5}=\left(i g_{s} / 2\right) G_{3} \wedge$ $\bar{G}_{3}=\left(i g_{s} / 4\right) d\left(A_{2} \wedge \bar{G}_{3}-\bar{A}_{2} \wedge G_{3}\right){ }^{13}$ This, of course, leads to the well-known expression $\tilde{F}_{5}=d C_{4}+\left(i g_{s} / 4\right)\left(A_{2} \wedge \bar{G}_{3}-\bar{A}_{2} \wedge G_{3}\right)$ with Chern-Simons terms acounting for the nontrivial Bianchi identity. The appearance of the 2 -form potentials requires $C_{4}$ to vary nontrivially under gauge transformations of $A_{2}$. This is the usual definition of the 4 -form potential in IIB SUGRA (one of the two common definitions, to be precise).

\footnotetext{
${ }^{13}$ When the axio-dilaton is constant; this discussion must be modified somewhat in a general $\mathrm{F}$ theory background.
} 
However, if $G_{3}$ has a harmonic background value $G_{3}^{(0)}$ (as in GKP compactifications), $A_{2}$ is defined only on coordinate patches, so $C_{4}$, including its fluctuation, must also be defined only in patches. Defining $G_{3}=G_{3}^{(0)}+\delta G_{3}$ with $\delta G_{3}=d \delta A_{2}$ exact, we have

$$
d \tilde{F}_{5}=\frac{i g_{s}}{2}\left[G_{3}^{(0)} \wedge \bar{G}_{3}^{(0)}+\delta G_{3} \wedge \bar{G}_{3}^{(0)}+G_{3}^{(0)} \wedge \delta \bar{G}_{3}+\delta G_{3} \wedge \delta \bar{G}_{3}\right] .
$$

Similarly splitting $\tilde{F}_{5}=\tilde{F}_{5}^{(0)}+\delta \tilde{F}_{5}$, where $\tilde{F}_{5}^{(0)}$ satisfies the Bianchi identity for $G_{3}=G_{3}^{(0)}$,

$$
d \delta \tilde{F}_{5}=\frac{i g_{s}}{2} d\left[\delta A_{2} \wedge \bar{G}_{3}^{(0)}-\delta \bar{A}_{2} \wedge G_{3}^{(0)}\right]+\frac{i g_{s}}{4} d\left[\delta A_{2} \wedge \delta \bar{G}_{3}-\delta \bar{A}_{2} \wedge \delta G_{3}\right] .
$$

This suggests writing

$$
\delta \tilde{F}_{5}=d \delta C_{4}^{\prime}+\frac{i g_{s}}{2}\left[\delta A_{2} \wedge \bar{G}_{3}^{(0)}-\delta \bar{A}_{2} \wedge G_{3}^{(0)}\right]+\frac{i g_{s}}{4}\left[\delta A_{2} \wedge \delta \bar{G}_{3}-\delta \bar{A}_{2} \wedge \delta G_{3}\right] .
$$

It is important to note, however, that $\delta C_{4}^{\prime}$ is not the fluctuation of $C_{4}$ as defined above but is shifted from that fluctuation by a wedge product of $\delta A_{2}$ and the patched 2-form potential that describes the background $G_{3}^{(0)}$. It is clear from (4.7) that $\delta C_{4}^{\prime}$ is a globally-defined form; [21, 25, 26] demonstrated this fact using the explicit field redefinition and the gauge transformations of the SUGRA fields.

Using the variables $\delta C_{4}^{\prime}, \delta A_{2}, \delta \bar{A}_{2}$ rather than $\delta C_{4}, \delta A_{2}, \delta \bar{A}_{2}$ serves two purposes: it removes the background gauge transformations from the first-order potential and clarifies the dependence of $\delta \tilde{F}_{5}$ on $\delta A_{2}, \delta \bar{A}_{2}$. Since $\delta C_{4}^{\prime}$ is globally-defined, it is the appropriate variable to describe fluctuations in moduli (such as 4-form axions) or compensators. However, because the explicit dependence of $\delta \tilde{F}_{5}$ on $\delta A_{2}$ changes, the field redefinition from $\delta C_{4}$ to $\delta C_{4}^{\prime}$ also modifies the 10D EOM for $\delta A_{2}$ compared to the usual result from the SUGRA (while leaving the 4-form EOM unchanged). As can be determined either by direct variation (at linear order) or by plugging the explicit field redefinition into (2.4), the change to the linearized form of (2.13) is to ensure that $A_{2}$ in the last term of that equation is the globally defined $\delta A_{2}$ (which may have a background value). Henceforth, we will use this modified potential and correspondingly modified EOM.

\subsubsection{Field redefinition for D3-brane source}

We can take a parallel approach for dynamical local sources; considering only a single D3-brane, the Bianchi identity (2.12) is

$$
d \tilde{F}_{5}=-2 \kappa_{10}^{2} T_{3} \int d^{4} \xi \sqrt{-\gamma} \star \epsilon_{\|} \delta^{10}(x, X(\xi)),
$$

which we evaluate in the static gauge $\partial_{a} X^{\mu}=\delta_{a}^{\mu}$. With this gauge choice, the integral reduces to

$$
\int d^{4} \xi \sqrt{-\gamma} \star \epsilon_{\|} \delta^{10}(x, X(\xi))=-\left(\star_{\perp}-\star_{\perp} \hat{d} Y_{1}+\frac{1}{2} \star_{\perp}\left(\hat{d} Y_{1} \wedge \hat{d} Y_{1}\right)+\cdots\right) \delta_{\perp}^{6}(y, Y(x))
$$

following from the definition (2.10). Remarkably, all factors of the metric cancel on both sides of $(4.9)$, so we can take $\star_{\perp}$ and $\delta_{\perp}^{6}$ to depend on an arbitrary $6 \mathrm{D}$ metric $g_{\perp, m n}$ on 
the $y^{m}$. For now we will leave this metric arbitrary, making an advantageous choice later. Because we can use an arbitrary $6 \mathrm{D}$ metric, our procedure does not rely on factorizability of the 10D metric. Note that explicit factors of parallel propagators contracting $\hat{d} Y$ have been suppressed since they become Kronecker deltas at coincidence (as enforced by the delta function). Notationally, we continue to define $\hat{d}=\partial_{\mu} d x^{\mu}, \tilde{d}=\partial_{m} d y^{m}$ rather than introduce $d_{\|}, d_{\perp}$.

Our approach, as when considering a fluctuating 3-form source in 4.2 .1 above, is to demonstrate that terms containing spacetime derivatives of the brane position are exact. Then $\tilde{F}_{5}$ can be written as the exterior derivative of a (redefined) potential plus deltafunction-supported terms making explicit the entire dependence of $\tilde{F}_{5}$ on the brane position $Y(x)$. To separate out the the dynamics of the brane position, we formally expand the right-hand side of (4.9) around a fixed arbitrary point $Y_{*}^{\underline{m}}$; note that this is not necessarily the background value of the D3-brane position. The proper expansion quantity is Synge's worldfunction $\sigma\left(Y_{*}, Y\right)$ (half the square geodesic distance between $Y_{*}^{m}$ and $\left.Y^{\not h}\right) .{ }^{14}$ The derivatives $\sigma_{\underline{m}} \equiv \partial_{\underline{m}} \sigma\left(Y_{*}, Y\right)$ lie tangent to the geodesic from $Y^{\text {th }}$ to $Y_{*}^{\underline{m}}$ in $T M_{Y_{*}}^{*}$ as illustrated in figure 2. By taking further partial derivatives, we can see that $\Lambda_{\eta p}^{m} \partial_{\mu} Y^{\eta h}=$ $-\Lambda_{\underline{m}}^{m} \partial_{\mu} \sigma^{\underline{m}}$, so we can replace $\hat{d} Y_{1} \rightarrow-\hat{d} \sigma_{1}$ in equation (4.9) above.

In carrying out this formal expansion, we note that

$$
\delta_{\perp}^{6}(y, Y)=\delta_{\perp}^{6}\left(y, Y_{*}\right)-\sigma^{\underline{m}} \partial_{\underline{m}} \delta_{\perp}^{6}\left(y, Y_{*}\right)+\frac{1}{2} \sigma^{\underline{m}} \sigma^{\underline{n}} \nabla_{\underline{m}} \partial_{\underline{n}} \delta_{\perp}^{6}\left(y, Y_{*}\right)+\mathcal{O}\left(\sigma^{3}\right) .
$$

Order by order in $\sigma$, then, we find

$$
\begin{aligned}
d \tilde{F}_{5}= & 2 \kappa_{10}^{2} T_{3}\left\{\epsilon_{\perp} \delta_{\perp}^{6}\left(y, Y_{*}\right)-\epsilon_{\perp} \sigma^{\underline{m}} \partial_{\underline{m}} \delta_{\perp}^{6}\left(y, Y_{*}\right)-\hat{d} \star_{\perp}\left(\sigma_{1} \delta_{\perp}^{6}\left(y, Y_{*}\right)\right)\right. \\
& \left.+\frac{1}{2} \epsilon_{\perp} \sigma^{\underline{m}} \sigma^{\underline{n}} \nabla_{\underline{m}} \partial_{\underline{n}} \delta_{\perp}^{6}\left(y, Y_{*}\right)+\left(\hat{d} \star_{\perp} \sigma_{1}\right) \sigma^{\underline{m}} \partial_{\underline{m}} \delta_{\perp}^{6}\left(y, Y_{*}\right)-\frac{1}{2} \star_{\perp}\left(\hat{d} \sigma_{1} \wedge \hat{d} \sigma_{1}\right) \delta_{\perp}^{6}\left(y, Y_{*}\right)\right\} .
\end{aligned}
$$

Using (B.5), and the fact that $\sigma^{\underline{m}}$ is function of $Y_{*}, Y$ and not the position $y$ where $\tilde{F}_{5}$ is evaluated, we can rewrite

$$
\sigma^{\underline{m}} \partial_{\underline{m}} \delta_{\perp}^{6}\left(y, Y_{*}\right)=-\nabla_{m}\left(\sigma^{\underline{m}} \Lambda_{\underline{m}}^{m} \delta_{\perp}^{6}\left(y, Y_{*}\right)\right)=\star_{\perp} \tilde{d} \star_{\perp}\left(\sigma_{1} \delta_{\perp}^{6}\left(y, Y_{*}\right)\right) .
$$

We immediately see that the second and third terms of (4.11) combine into a total derivative $-d\left(\star_{\perp} \sigma_{1} \delta_{\perp}^{6}\left(y, Y_{*}\right)\right)$.

We wish to write the second order terms also as 10D total derivatives. We start by noting that similarly

$$
\star_{\perp} \sigma^{\underline{m}} \sigma^{\underline{n}} \nabla_{\underline{m}} \partial_{\underline{n}} \delta_{\perp}^{6}\left(y, Y_{*}\right)=\tilde{d}\left[\left(\star_{\perp} \sigma_{1}\right) \sigma^{\underline{m}} \partial_{\underline{m}} \delta_{\perp}^{6}\left(y, Y_{*}\right)\right]
$$

and also

$$
\star_{\perp}\left(\hat{d} \sigma_{1} \wedge \hat{d} \sigma_{1}\right) \delta_{\perp}^{6}\left(y, Y_{*}\right)=\hat{d}\left[\star_{\perp}\left(\sigma_{1} \wedge \hat{d} \sigma_{1}\right) \delta_{\perp}^{6}\left(y, Y_{*}\right)\right] .
$$

\footnotetext{
${ }^{14}$ For additional details, see appendix B.
} 
The remaining term in (4.11) can be re-written in two ways; the first is given by the simple differentiation

$$
\hat{d}\left[\left(\star_{\perp} \sigma_{1}\right) \sigma^{\underline{m}} \partial_{\underline{m}} \delta_{\perp}^{6}\left(y, Y_{*}\right)\right]=\left(\hat{d} \star_{\perp} \sigma_{1}\right) \sigma^{\underline{m}} \partial_{\not h} \delta_{\perp}^{6}\left(y, Y_{*}\right)-\left(\star_{\perp} \sigma_{1}\right) \hat{d} \sigma^{\underline{m}} \partial_{\underline{m}} \delta_{\perp}^{6}\left(y, Y_{*}\right) .
$$

While the left-hand side of (4.15) looks like the complement to (4.13) that we desire, it enters (4.11) with an incorrect coefficient, and the third term of (4.15) is not a derivative. As it turns out, the complement of (4.14) is

$$
\tilde{d}\left[\star_{\perp}\left(\sigma_{1} \wedge \hat{d} \sigma_{1}\right) \delta_{\perp}^{6}\left(y, Y_{*}\right)\right]=-\hat{d}\left(\star_{\perp} \sigma_{1}\right) \sigma^{\underline{m}} \partial_{\underline{m}} \delta_{\perp}^{6}\left(y, Y_{*}\right)-\left(\star_{\perp} \sigma_{1}\right) \hat{d} \sigma^{\underline{m}} \partial_{\underline{m}} \delta_{\perp}^{6}\left(y, Y_{*}\right),
$$

where we have remembered to differentiate the parallel propagators $\Lambda_{\underline{m}}^{m}$ in the definition of $\sigma_{1}$. All told,

$$
\hat{d}\left(\star_{\perp} \sigma_{1}\right) \sigma^{\underline{m}} \partial_{\underline{m}} \delta_{\perp}^{6}\left(y, Y_{*}\right)=\frac{1}{2} \hat{d}\left[\left(\star_{\perp} \sigma_{1}\right) \sigma^{\underline{m}} \partial_{\underline{m}} \delta_{\perp}^{6}\left(y, Y_{*}\right)\right]-\frac{1}{2} \tilde{d}\left[\star_{\perp}\left(\sigma_{1} \wedge \hat{d} \sigma_{1} \delta_{\perp}^{6}\left(y, Y_{*}\right)\right],\right.
$$

so, to second order in the formal expansion,

$$
\begin{aligned}
d \tilde{F}_{5}= & 2 \kappa_{10}^{2} T_{3}\left\{\epsilon_{\perp} \delta_{\perp}^{6}\left(y, Y_{*}\right)-d\left[\star_{\perp} \sigma_{1} \delta_{\perp}^{6}\left(y, Y_{*}\right)\right]+\frac{1}{2} d\left[\left(\star_{\perp} \sigma_{1}\right) \sigma^{\underline{m}} \partial_{\underline{m}} \delta_{\perp}^{6}\left(y, Y_{*}\right)\right]\right. \\
& \left.-\frac{1}{2} d\left[\star_{\perp}\left(\sigma_{1} \wedge \hat{d} \sigma_{1}\right) \delta_{\perp}^{6}\left(y, Y_{*}\right)\right]\right\} .
\end{aligned}
$$

While we do not carry out this calculation to higher order, we conjecture that all terms in the Bianchi identity at first or higher order in the formal $\sigma$ expansion can be organized into total derivatives, as we have shown at first and second order. It is important to note that the source terms are actually independent of $Y_{*}$ when all orders of the expansion are included, since they are simply a way of re-writing a function of $Y(x)$.

Since the source for the Bianchi identity is a static delta function plus a series of total derivatives according to our conjecture, $\tilde{F}_{5}$ can be written in terms of a patched-together potential for a static magnetic monopole located at $Y_{*}$, a globally-defined potential, and additional terms that translate the Dirac string from $Y_{*}^{\underline{m}}$ to $Y^{m}$ as

$$
\begin{aligned}
\tilde{F}_{5}= & d S_{4}^{*}+d C_{4}^{\prime}-2 \kappa_{10}^{2} T_{3}\left[\star_{\perp} \sigma_{1} \delta_{\perp}^{6}\left(y, Y_{*}\right)-\frac{1}{2}\left(\star_{\perp} \sigma_{1}\right) \sigma^{\underline{m}} \partial_{\underline{m}} \delta_{\perp}^{6}\left(y, Y_{*}\right)\right. \\
& \left.+\frac{1}{2} \star_{\perp}\left(\sigma_{1} \wedge \hat{d} \sigma_{1}\right) \delta_{\perp}^{6}\left(y, Y_{*}\right)+\cdots\right] .
\end{aligned}
$$

Note that the static monopole potential $S_{4}^{*}$ satisfies $d^{2} S_{4}^{*}=2 \kappa_{10}^{2} T_{3} \star_{\perp} \delta_{\perp}^{6}\left(y, Y_{*}\right)$, which is allowed since it is not globally defined and is singular at $y=Y_{*}$. Of course, $\tilde{F}_{5}$ and all physically meaningful quantities must be independent of the arbitrarily chosen $Y_{*}$, so the equation $\partial \tilde{F}_{5} / \partial Y_{*}^{\underline{m}}=0$ will result in a system of relations among different orders of the expansion similar to renormalization group flow.

We emphasize that (4.19) is not our ansatz for D3-brane motion. Rather, any 5-form field strength with a monopole source can be written in this form, as we have done for our ansatz in (4.3). Specifically, $\delta \tilde{F}_{5}$ in that expression is the first order term of $\tilde{F}_{5}$ in 
the fluctuation of the brane position; to make contact with that expression, we should take $Y_{*}$ to be the fixed background brane position, $Y$ the actual brane position including fluctuations, and $g_{\perp, m n}=\tilde{g}_{m n}$. Then $d S_{4}^{*}+d C_{4}^{\prime}$ is the background 5 -form, and $\sigma_{1} \rightarrow-\tilde{Y}_{1}$. Then (4.19) is identically (4.3) to first order.

Just as in section 4.2.1, the field redefinition from $C_{4} \rightarrow C_{4}^{\prime}$ serves a dual purpose. First, it is clear that $d^{2} C_{4}^{\prime}=0$, so, unlike the original 4 -form, the redefined potential is now globally defined and is suitable for dimensional reduction. Therefore, the $C_{4}$ compensators and axion moduli appear in the redefined $C_{4}^{\prime}$. Further, the field redefinition cleanly separates $\tilde{F}_{5}$ into a contribution from the independent 4 -form potential $C_{4}^{\prime}$ and an explicit contribution from the D3-brane degrees of freedom (which is required by the Bianchi identity). As for other moduli, the explicit dependence of the field strength on $Y(x)$ must be supplemented by the appearance of compensators, which are necessary to satisfy the constraints. In addition, since $\tilde{F}_{5}$ explicitly depends on the brane position when written in terms of $C_{4}^{\prime}$, the D3-brane EOM is not simply given by the DBI and WZ actions, as we discuss below.

\subsubsection{The brane EOM in the magnetic picture}

As we have noted previously, in the magnetic version of IIB SUGRA, D3-branes couple to $\tilde{F}_{5}$ only through the nontrivial Bianchi identity; there is no WZ coupling between $C_{4}$ and a static D3-brane. This naively presents a puzzle, since the brane is mutually BPS with a GKP background (or the background of other static D3-branes) and should therefore feel no force. The DBI action provides a gravitational force, but there is apparently no counter-balancing force from the 5 -form, unlike in the electric formulation of the theory!

The resolution of the puzzle lies in the redefinition of $\tilde{F}_{5}$ in (4.19). While the redefinition leaves the $\tilde{F}_{5}$ EOM $E_{6}$ unchanged because $C_{4}^{\prime}$ enters $\tilde{F}_{5}$ in the same way as $C_{4}$ does, the new explicit dependence of $\tilde{F}_{5}$ on the brane position $Y^{\eta h}$ through $\sigma_{\underline{m}}\left(Y_{*}, Y\right)$ modifies the D3-brane's EOM, just as the SUGRA Chern-Simons terms in $\tilde{F}_{5}$ contribute to the EOM for $A_{2}$. We show here that the delta-function-supported terms in equation (4.19) introduce two new contributions to the brane EOM: a force, which resolves the puzzle described above, and terms proportional to the 5 -form EOM $E_{6}$, which vanish on shell but will contribute to off-shell quantities including the effective action.

In the magnetic description, the D3-brane position degrees of freedom appear in the DBI action (2.7) and the $\tilde{F}_{5}$ kinetic terms, which are

$$
S_{5}=-\left.\frac{1}{2 \kappa_{10}^{2}} \int d^{10} x \sqrt{-g}\left(\frac{1}{2}\right)\left(\frac{1}{5 !}\right) \tilde{F}_{M N P Q R} \tilde{F}^{M N P Q R}\right|_{\mathrm{mag}},
$$

where the subscript mag indicates that the sum is over only the magnetic components of $\tilde{F}_{5}$ (which are defined with indices lowered). Note that the indices are raised with the full $10 \mathrm{D}$ metric $g^{M N}$. In the static gauge, the pullback of the metric is

$$
P(g)_{\mu \nu}=g_{\mu \nu}(x, Y)+2 g_{\mu \hbar}(x, Y) \hat{\partial}_{\nu} Y^{\not h}+g_{\eta \not h}(x, Y) \hat{\partial}_{\mu} Y^{\not h} \hat{\partial}_{\nu} Y^{\not h} ;
$$

the EOM for $\gamma_{\mu \nu}$ also enforce $\gamma_{\mu \nu}=P(g)_{\mu \nu}$, but we impose that constraint only after varying $S$ with respect to $Y^{\eta}$. The DBI part of the action, as previously, contributes 
terms equal to the $\operatorname{EOM}(2.14)$ with $C_{M I N D Q}=0$ and all indices $X^{M I}$ restricted to $Y^{\not h}$ in static gauge.

We now determine the variation of the 5 -form kinetic action with respect to the brane position. To first order in the $\sigma$ expansion,

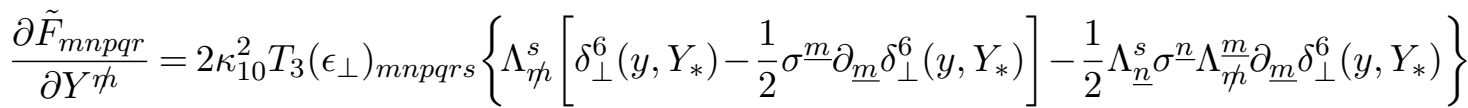

$$
\begin{aligned}
& =2 \kappa_{10}^{2} T_{3}\left(\epsilon_{\perp}\right)_{m n p q r s}\left\{\Lambda_{m p}^{s} \delta_{\perp}^{6}(y, Y)+\Lambda_{\underline{s}}^{s} \Lambda_{\eta \underline{h}}^{[\underline{s}} \sigma^{\underline{m}]} \partial_{\underline{m}} \delta_{\perp}^{6}\left(y, Y_{*}\right)\right\} \text {, } \\
& \frac{\partial \tilde{F}_{\mu m n p q}}{\partial Y^{\eta p}}=\kappa_{10}^{2} T_{3}\left(\epsilon_{\perp}\right)_{m n p q r s} \Lambda_{\eta h}^{r} \Lambda_{\not h}^{s} \partial_{\mu} Y^{\not h} \delta_{\perp}^{6}\left(y, Y_{*}\right) \text {, } \\
& \frac{\partial \tilde{F}_{\mu m n p q}}{\partial\left(\hat{\partial}_{\nu} Y^{\eta h}\right)}=-\kappa_{10}^{2} T_{3} \delta_{\mu}^{\nu}\left(\epsilon_{\perp}\right)_{m n p q r s} \Lambda_{\underline{m}}^{r} \sigma^{\underline{m}} \Lambda_{m p}^{s} \delta_{\perp}^{6}\left(y, Y_{*}\right) \text {. }
\end{aligned}
$$

We have used the relationship $\partial \sigma^{\underline{m}} / \partial Y^{\not h}=-\Lambda \frac{m}{\eta_{h}}$ as well as the expansion (4.10) for the delta function.

For the most part, the EOM can be evaluated using the typical Euler-Lagrange formula. However, the contribution from the second term of (4.22) deserves special consideration. In the variation of the action, we can integrate by parts to remove the derivative from the delta function:

$$
\delta S_{5}=T_{3} \int d^{10} x \frac{1}{5 !} \delta Y^{\eta h}\left\{\nabla_{t}^{\perp}\left[\sqrt{-g}\left(\epsilon_{\perp}\right)_{m n p q r s} \tilde{F}^{m n p q r} \Lambda_{\underline{s}}^{s}\right] \Lambda_{m}^{[\underline{s}} \sigma^{\underline{m}]} \Lambda_{\underline{m}}^{t} \delta_{\perp}^{6}\left(y, Y_{*}\right)+\cdots\right\},
$$

where $\nabla^{\perp}$ is the covariant derivative compatible with the as yet arbitrary metric $g_{\perp, m n}$. With the antisymmetrization of the $\underline{s}, \underline{m}$ indices, the derivative appears to be $\tilde{d} \star_{\perp} \tilde{F}_{5}$; however, the presence of the 10D metric requires a more delicate interpretation. As we expect that the brane EOM will contain terms proportional to the $\tilde{F}_{5}$ EOM, we are motivated to re-write

$$
\frac{1}{5 !} \nabla_{[t}^{\perp}\left[\sqrt{-g}\left(\epsilon_{\perp}\right)_{|m n p q r| s]} \tilde{F}^{m n p q r}\right] \Lambda_{\underline{n}}^{t} \Lambda_{\eta h}^{s}=\frac{1}{2} \sqrt{-\gamma} \sqrt{g_{\perp}}(\star \gamma \tilde{d} \star \tilde{F})_{\underline{n} \eta \underline{h}},
$$

where $\star_{\gamma}$ is the $4 \mathrm{D}$ Hodge star for the induced metric; this combination is ultimately independent of $\gamma_{\mu \nu} \cdot{ }^{15}$ Note that only certain components of $\tilde{F}$ appear in $\left(\star_{\gamma} \tilde{d} \star \tilde{F}\right)_{t s}$. Similarly, the $\partial_{\mu}\left(\partial \mathcal{L} / \partial\left(\partial_{\mu} Y\right)\right)$ term of the Euler-Lagrange equation contains

$$
\frac{1}{4 !} \partial_{\mu}\left[\sqrt{-g}\left(\epsilon_{\perp}\right)_{m n p q r s} \tilde{F}^{\mu m n p q} \Lambda_{\eta p}^{s}\right] \Lambda_{\underline{n}}^{n}=\sqrt{-\gamma} \sqrt{g_{\perp}} \star \gamma \hat{d} \star \tilde{F}_{\underline{n} \not h}
$$

(with a slight abuse of notation). These terms in fact add together to give a contribution proportional to the EOM $E_{6}$.

\footnotetext{
${ }^{15}$ Recall that $g_{M N}$ and $\gamma_{\mu \nu}$ are independent variables until the $\gamma_{\mu \nu}$ EOM is enforced.
} 
All told, the variation of the action with respect to the brane position (in static gauge) is

$$
\begin{aligned}
& \delta S=-T_{3} \int d^{10} x \sqrt{-g} \delta Y^{\not h}\left\{( \frac { 1 } { 2 } ) \int d ^ { 4 } \xi \sqrt { - \gamma } \left[\gamma ^ { \mu \nu } \left(\partial_{\eta \downarrow} g_{\mu \nu}(x, Y)+2 \partial_{\eta h} g_{\not h(\mu} \partial_{\nu)} Y^{\not h}\right.\right.\right. \\
& \left.\left.+\partial_{\not h} g_{\not h p h} \partial_{\mu} Y^{\not h} \partial_{\nu} Y^{\not p}\right)-2 \nabla_{\mu}^{\gamma}\left(\gamma^{\mu \nu} g_{\eta m h} \partial_{\nu} Y^{\not h}+\gamma^{\mu \nu} g_{\nu \not h}\right)\right] \delta^{10}(x, X) \\
& -\left[\frac{1}{5 !}\left(\epsilon_{\perp}\right)_{\eta n n p q r s} \tilde{F}^{n p q r s} \delta_{\perp}^{6}(y, Y)-\frac{1}{2} \frac{\sqrt{-\gamma} \sqrt{g_{\perp}}}{\sqrt{-g}}\left(\star_{\gamma} d \star \tilde{F}_{5}\right)_{\underline{n} \eta \underline{n}} \sigma^{\underline{n}} \delta_{\perp}^{6}\left(y, Y_{*}\right)\right. \\
& \left.\left.+\frac{1}{5 !}\left(\epsilon_{\perp}\right)_{\not h p p q r s} \tilde{F}^{\mu p q r s} \partial_{\mu} Y^{h t} \delta_{\perp}^{6}\left(y, Y_{*}\right)\right]\right\} \text {. }
\end{aligned}
$$

We need to make several comments. First, we have worked only to the first subleading order in the formal $\sigma$ expansion. It is reasonable to conjecture that the sole effect of the higher order terms in $\sigma$ is to replace $\delta_{\perp}^{6}\left(y, Y_{*}\right) \rightarrow \delta_{\perp}^{6}(y, Y)$ and perhaps to add new contributions to the EOM which do not contribute at first order in the D3-brane velocity (like the last term of (4.28)). We will primarily assume that this is the case in our discussion of the dimensionally reduced action below but also comment on the possibility that the conjecture is false (we leave a check of the conjecture to [29]). Next, we note the appearance of several different metrics in (4.28) including the (as yet) arbitrary metric $g_{\perp, m n}$. As we mentioned above, this formalism has allowed us to include geometries in which $g_{M N}$ does not factorize into $4 \mathrm{D}$ and $6 \mathrm{D}$ metrics. Finally, we recall from equations (2.4), (2.14) our convention that the EOM should be defined as (in static gauge)

$$
\delta S=T_{3} \int d^{10} x \sqrt{-g} \int d^{4} \xi \sqrt{-\gamma} \delta Y^{\eta h} E_{m h} \text { with } E_{m h} \propto \delta^{10}(x, X) .
$$

Some of the terms in (4.28) manifestly take this form, but others do not. However, consider that

$$
\int d^{10} x \sqrt{-g} \int d^{4} \xi \sqrt{-\gamma} f(x, y, Y) \delta^{10}(x, X)=\int d^{10} x \sqrt{-\gamma} \sqrt{g_{\perp}} f(x, y, Y) \delta_{\perp}^{6}(y, Y)
$$

for any function $f(x, y, Y)$. Furthermore, we have the identities $\sqrt{-\gamma} \sqrt{g_{\perp}} \epsilon_{\gamma}^{\mu \nu \lambda \rho} \epsilon_{\mu \nu \lambda \rho m n p q r s}$ $=-4 ! \sqrt{-g}\left(\epsilon_{\perp}\right)_{m n p q r s}$ and $\sqrt{-\gamma} \sqrt{g_{\perp}} \epsilon_{\gamma}^{\mu \lambda \rho \sigma} \epsilon_{\nu \lambda \rho \sigma m n p q r s}=-6 \sqrt{-g} \delta_{\nu}^{\mu}\left(\epsilon_{\perp}\right)_{\text {mnpqrs }}$. Therefore, we can re-write (4.28) as the EOM

$$
\begin{aligned}
& E_{\not h}=\left\{\nabla_{\mu}^{\gamma}\left(\gamma^{\mu \nu} g_{\not h \not h} \partial_{\nu} Y^{\not h}+\gamma^{\mu \nu} g_{\nu \not h}\right)-\frac{1}{2} \gamma^{\mu \nu}\left(\partial_{\not h} g_{\mu \nu}+2 \partial_{\eta h} g_{\not h(\mu} \partial_{\nu)} Y^{\not h}\right.\right. \\
& \left.+\partial_{\not h} g_{\not h p} \partial_{\mu} Y^{h} \partial_{\nu} Y^{\not p}\right)-\frac{1}{2}\left(\star_{\gamma} d \star \tilde{F}_{5}\right)_{n \eta \underline{h}} \Lambda_{\underline{n}}^{n} \sigma^{\underline{n}} \\
& \left.-\left(\left(\star_{\gamma} \star \tilde{F}_{5}\right)_{\eta h}+\left(\star_{\gamma} \star \tilde{F}_{5}\right)^{\mu}{ }_{\eta \eta \eta} \partial_{\mu} Y^{\not h}\right)\right\} \delta^{10}(x, X) \text {. }
\end{aligned}
$$

Note that the final result depends only on the 10D metric and the induced metric on the worldvolume, not the arbitrary metric $g_{\perp, m n}$; this is physically necessary but occurs through nontrivial cancellations. The field redefinition $C_{4} \rightarrow C_{4}^{\prime}$ which showed the explicit dependence of $\tilde{F}_{5}$ on $Y^{\eta h}$ has introduced several new terms in the D3-brane EOM. The last two terms represent the "electromagnetic Lorentz" force of $\tilde{F}_{5}$ on the brane and, at least to 
the order we have calculated in $\sigma$, they are independent of the arbitrary reference point $Y_{*}$, as any physical quantity should be. Higher orders in the $\sigma$ expansion can contribute terms with higher powers of $\partial_{\mu} Y^{\not h}$ as necessary for the 5 -form version of the Lorentz force. The remaining term, proportional to $E_{6}=d \star \tilde{F}_{5}$, is somewhat more puzzling because it contains $\sigma^{\underline{n}}$. This term vanishes on shell, so it does not affect the physically meaningful brane EOM, but it does contribute to the (off-shell) quadratic action that we wish to calculate. We will discuss this contribution in more detail when we calculate the quadratic action below. For now, we simply note that we can replace $\left(\star_{\gamma} E_{6}\right)_{n \eta \underline{h}} \Lambda_{\underline{r}}^{n} \sigma^{\underline{n}}=\left(\star_{\gamma} E_{6}\right)_{\eta \eta_{n}} \sigma^{h /}$ using properties of the Synge world function (see appendix B).

We can now return to the puzzle we raised earlier — how does a static magneticallycharged brane feel a BPS-like no-force condition even though it has no direct coupling to the 5 -form? We have recognized that the nontrivial Bianchi identity for $\tilde{F}_{5}$ modifies the D3-brane EOM as above. Consider a D3-brane in the background (2.2) at a constant position $Y^{\eta h}$. In this case, $\gamma_{\mu \nu}=g_{\mu \nu}=e^{2 A} \hat{\eta}_{\mu \nu}$. Then the brane EOM becomes

$$
\begin{aligned}
E_{\not h} & =\left[-\frac{1}{2} \gamma^{\mu \nu} \partial_{\not h} g_{\mu \nu}+\left(\star_{\gamma} \star \tilde{F}_{5}\right)_{\not h}\right] \delta^{10}(x, X) \\
& =\left[-2 e^{-2 A} \partial_{\not h} e^{2 A}-e^{-6 A} e^{10 A} \partial_{\not h} e^{-4 A}\right] \delta^{10}(x, X)=0 .
\end{aligned}
$$

In other words, the new contributions to the brane EOM due to the $\tilde{F}_{5}$ field redefinition precisely restore the no-force condition for the brane.

It is also worth discussing the relationship of (4.31) to the D3-brane EOM in the electric formalism as given in (2.14). In static gauge, ${ }^{16}$ this is

$$
\begin{aligned}
E_{\not h}= & \left\{\nabla_{\mu}^{\gamma}\left(\gamma^{\mu \nu} g_{\not h n} \partial_{\nu} Y^{\not h}+\gamma^{\mu \nu} g_{\nu \not h}\right)-\frac{1}{2} \gamma^{\mu \nu}\left(\partial_{\eta h} g_{\mu \nu}+2 \partial_{\not h} g_{\not h(\mu} \partial_{\nu)} Y^{\not h}\right.\right. \\
& \left.+\partial_{\not h} g_{\not h p p} \partial_{\mu} Y^{\not h} \partial_{\nu} Y^{\not p}\right)+\epsilon_{\gamma}^{\mu \nu \lambda \rho} \nabla_{\mu}^{\gamma}\left(\frac{1}{6} C_{\not h \nu \lambda \rho}+\frac{1}{2} C_{\not h \eta h \lambda \rho} \partial_{\nu} Y^{\not h}+\cdots\right) \\
& \left.-\epsilon_{\gamma}^{\mu \nu \lambda \rho}\left(\frac{1}{24} \partial_{\not h} C_{\mu \nu \lambda \rho}+\frac{1}{6} \partial_{\not h} C_{\not h \nu \lambda \rho} \partial_{\mu} Y^{\not h}+\cdots\right)\right\} \delta^{10}(x, X),
\end{aligned}
$$

where the $\cdots$ include higher powers of $\partial_{\mu} Y^{\eta h}$, which we did not calculate in the magnetic framework. The first terms, which involve the metric and its derivatives, are manifestly identical in the electric and magnetic formalisms, so we are left to compare the terms involving the potential/flux. With some rearrangement,

$$
\frac{1}{6} \epsilon_{\gamma}^{\mu \nu \lambda \rho} \nabla_{\mu}^{\gamma} C_{\not h \nu \lambda \rho}=-\frac{1}{24} \epsilon_{\gamma}^{\mu \nu \lambda \rho}\left(\hat{d} C_{4}\right)_{\mu \nu \lambda \rho \not h}+\frac{1}{6} \epsilon_{\gamma}^{\mu \nu \lambda \rho} \partial_{\not h} C_{\not h \nu \lambda \rho} \partial_{\mu} Y^{\not h}
$$

the first term combines with the first term of the third line of $(4.33)$ to give $-\left(\star_{\gamma} \tilde{F}_{5}\right)_{\not h}$ in terms of the electric components. We add the remainder to the other terms from (4.33) and find

$$
-\frac{1}{3} \epsilon_{\gamma}^{\mu \nu \lambda \rho} \partial_{[\not h} C_{\not h] \nu \lambda \rho} \partial_{\mu} Y^{\not h}+\frac{1}{2} \epsilon_{\gamma}^{\mu \nu \lambda \rho} \nabla_{\mu}^{\gamma} C_{\not h \not \lambda \rho} \partial_{\nu} Y^{\not h}=-\left(\star_{\gamma} \tilde{F}_{5}\right)^{\mu}{ }_{\not h \eta} \partial_{\nu} Y^{\not h}+\cdots
$$

\footnotetext{
${ }^{16}$ And excluding terms proportional to derivatives of the delta function as in footnote 6 .
} 
after remembering the no-torsion condition for derivatives of scalars. As when deriving the D3-brane EOM originally, we have assumed that the 2-form potentials and 3-form fluxes do not contribute to the electric components of $\tilde{F}_{5}$. Now we see that the D3-brane EOM in the electric and magnetic formalisms are equivalent (at least the terms we consider) if

$$
\left[\left(\star_{\gamma} \tilde{F}_{5}\right)_{\not h}+\left(\star_{\gamma} \tilde{F}_{5}\right)^{\mu}{ }_{\eta \eta h} \partial_{\nu} Y^{\not h}\right]^{\text {elec }}=\left[\left(\star_{\gamma} \star \tilde{F}_{5}\right)_{\eta_{h}}+\left(\star_{\gamma} \star \tilde{F}_{5}\right)^{\mu}{ }_{\eta_{\eta h}} \partial_{\nu} Y^{\not h}\right]^{\text {mag }},
$$

which is simply the relation of the electric and magnetic components of the fieldstrength to each other.

\subsubsection{Summary}

In the presence of a nontrivial Bianchi identity, the potential $C_{4}$ as usually defined contains both an independent degree of freedom in the 10D SUGRA (which contributes both to the 4 -form axions and compensators of other moduli in dimensional reduction) and also a direct dependence on the D3-brane position. This potential carries a Dirac string singularity which moves with the brane (alternately described as gauge patching); because of the nonstandard periodicity conditions, $C_{4}$ is not appropriate to describe moduli or compensators in dimensional reduction, and it also does not accurately reflect the contribution of the brane position to the action.

As we describe in section 4.2.1, this situation is similar to the case of background 3 -form flux; we reviewed a field transformation previously described by $[21,25,26]$ to a globally defined 4 -form $C_{4}^{\prime}$ which also makes explicit the dependence of $\tilde{F}_{5}$ on fluctuations in $A_{2}$. We then found that it is similarly possible to write $\tilde{F}_{5}$ in the presence of a D3-brane monopole as

$$
\begin{aligned}
\tilde{F}_{5}= & d S_{4}^{*}+d C_{4}^{\prime}-2 \kappa_{10}^{2} T_{3}\left[\star_{\perp} \sigma_{1} \delta_{\perp}^{6}\left(y, Y_{*}\right)-\frac{1}{2}\left(\star_{\perp} \sigma_{1}\right) \sigma^{\underline{m}} \partial_{\underline{m}} \delta_{\perp}^{6}\left(y, Y_{*}\right)\right. \\
& \left.+\frac{1}{2} \star_{\perp}\left(\sigma_{1} \wedge \hat{d} \sigma_{1}\right) \delta_{\perp}^{6}\left(y, Y_{*}\right)+\cdots\right]
\end{aligned}
$$

in terms of a new potential $C_{4}^{\prime}$, which is globally defined and an independent degree of freedom.

The explicit dependence of $\tilde{F}_{5}$ on the brane position contributes to the brane EOM, as derived in (4.28). The modified EOM satisfies the no-force condition on a static D3brane in a GKP background, which would otherwise be a mystery due to the lack of a WZ coupling to the magnetic 4-form.

\subsection{D effective action in magnetic formalism}

In this section, we calculate the $4 \mathrm{D}$ effective action in the magnetic formulation of the SUGRA. We begin by reviewing the ansätze for the D3-brane positions, volume modulus, and axions, and then we compute the quadratic action and integrate over the internal manifold. Appendix C.2 contains details of the calculation. 


\subsubsection{Kähler moduli in magnetic components}

The 10D ansätze (and solutions to the constraints) for the volume modulus and $C_{4}$ axions in terms of the magnetic components of $\tilde{F}_{5}$ were presented in $[25,26]$. We review those results here in concert with the ansatz for D3-brane motion as given in section 4.1.

All these moduli are described by the same metric ansatz (3.15) as in the electric formulation, including a compensator field

$$
B_{1}=-c(x) \tilde{d} K(y)+b_{0}^{I}(x) B_{1}^{I}(y)+B_{1}^{Y}(x, y) .
$$

The 4-form perturbation is

$$
\delta C_{4}^{\prime}=b_{0}^{I}(x) \tilde{\star} \omega_{2}^{I}(y)-\hat{d} b_{0}^{I} K_{3}^{I}(y)-e^{2 \Omega} \tilde{\star} \tilde{d} B_{1}^{Y}(x, y)
$$

note that the volume modulus does not appear in the magnetic components of $C_{4}^{\prime}$, even through its compensator. There is an additional compensator for the axions in the 2 -form potential, $\delta A_{2}=-\hat{d} b_{0}^{I}(x) \Lambda_{1}^{I}(y)$, which is nontrivial only in the presence of a background 3 -form flux. Altogether, the field strengths are

$$
\begin{aligned}
\tilde{F}_{5}= & \tilde{\star} \tilde{d} e^{-4 A}-e^{2 \Omega} \hat{d}\left(\tilde{\star} \tilde{d} B_{1}^{Y}\right)+\hat{d} b_{0}^{I} \wedge\left(\tilde{\star} \omega_{2}^{I}+\tilde{d} K_{3}^{I}-\frac{i g_{s}}{2}\left(\Lambda_{1}^{I} \wedge \bar{G}_{3}^{(0)}-\bar{\Lambda}_{1}^{I} \wedge G_{3}^{(0)}\right)\right) \\
& +\left[e^{4 \Omega} \hat{\epsilon} \wedge \tilde{d} e^{4 A}-e^{4 \Omega} \hat{\star} \hat{d} \tilde{d}\left(e^{4 A} B_{1}\right)+e^{2 \Omega} \hat{\star} \hat{d} b_{0}^{I} \wedge \gamma_{2}^{I}\right] \text { and } \\
G_{3}= & G_{3}^{(0)}+\hat{d} b_{0}^{I} \wedge \tilde{d} \Lambda_{1}^{I} .
\end{aligned}
$$

The field strength $\tilde{F}_{5}$ is not the background plus $d \delta C_{4}^{\prime}$ because $\tilde{F}_{5}$ includes extra terms as in (4.7), (4.19).

The compensators and warp factor are given by equations (3.23), (3.24), (3.11), as in the electric formulation, along with

$$
\tilde{\nabla}^{2} \Lambda_{m}^{I}=-\frac{1}{2} G_{m n p}^{(0)} \gamma^{I, \widetilde{n p}}, \quad \tilde{\nabla}^{2} B_{m}^{I}=-e^{-2 \Omega} \gamma_{m n}^{I} \partial^{\tilde{n}} e^{-4 A}-\frac{i g_{s}}{2} \tilde{\star}\left(\tilde{d} \Lambda_{1}^{I} \wedge \bar{G}_{3}^{(0)}-\text { c.c. }\right)_{m}
$$

for the axions. The new form $\gamma_{2}^{I}$ in (4.39), (4.41) is harmonic (with the CY metric $\tilde{g}_{m n}$ ) satisfying

$$
\gamma_{2}^{I} \equiv e^{4 A}\left[\omega_{2}^{I}+\tilde{\star}\left(\tilde{d} K_{3}^{I}-\frac{i g_{s}}{2}\left(\tilde{d} \Lambda_{1}^{I} \wedge \bar{G}_{3}^{(0)}-\text { c.c. }\right)\right)+e^{2 \Omega} \tilde{d} B_{1}^{I}\right] \equiv C^{I J} \omega_{2}^{J} .
$$

The matrix $C^{I J}$ is defined as in (3.38); see [26] for more details of the axion degrees of freedom. The final constraint can be written conveniently as

$$
\tilde{\nabla}^{\tilde{m}} B_{m}=e^{-2 \Omega} \delta e^{-4 A}-e^{-4 A} \delta e^{-2 \Omega},
$$

for the total compensator (4.37) including the variations due to all the moduli; the compensator $B_{1}^{I}$ for the axions is divergenceless. 


\subsubsection{Effective action}

The contribution to the quadratic action from the Einstein equations is only through the $(m n)$ component. By contracting the first-order parts of

$$
\delta E_{m n}=\hat{\partial}^{2}\left[4 \partial_{(m} A B_{n)}-2 \tilde{g}_{m n} \partial^{\tilde{p}} A B_{p}+\tilde{\nabla}_{(m} B_{n)}-\frac{1}{2} e^{-2 \Omega} e^{-4 A} \tilde{g}_{m n}\right]
$$

with

$$
\delta g^{m n}=\delta\left(e^{2 A} \tilde{g}^{m n}\right)=-\frac{1}{2} e^{6 A} \delta e^{-4 A} \tilde{g}^{m n},
$$

we arrive at

$$
S_{\text {eff }}^{R}=-\frac{1}{8 \kappa_{10}^{2}} \int d^{4} x \int d^{6} y \sqrt{\tilde{g}} e^{4 \Omega}\left[-6 \delta e^{-4 A} \hat{\partial}^{2} c+2 e^{4 A} \partial^{\tilde{m}} \delta e^{-4 A} \hat{\partial}^{2} B_{m}\right]
$$

after integration by parts and use of (4.43). Note that $\delta e^{-2 \Omega}=c(x)$, the volume modulus. The first term of (4.46) is proportional to

$$
\int d^{6} y \sqrt{\tilde{g}} \delta e^{-4 A}=\tilde{V} c(x)
$$

because the brane motion does not change the warped volume per (3.8). Therefore,

$$
S_{\text {eff }}^{R}=\frac{1}{4 \kappa_{10}^{2}} \int d^{4} x e^{4 \Omega}\left[3 \tilde{V} c \hat{\partial}^{2} c-\int d^{6} y \sqrt{\tilde{g}} e^{4 A} \partial^{\tilde{m}} \delta e^{-4 A} \hat{\partial}^{2} B_{m}\right] .
$$

Next, we examine the contribution from the 5-form EOM. The first-order parts of $E_{6}$ include the dynamical EOM for the axion as well as a contribution from the compensator for all moduli:

$$
\delta E_{6}=e^{2 \Omega} \hat{d} \hat{\star} \hat{d} b_{0}^{I} \wedge \gamma_{2}^{I}-e^{4 \Omega} \hat{d} \hat{\star} \hat{d} \tilde{d}\left(e^{4 A} B_{1}\right) .
$$

As in (2.4), we wedge this with $\delta C_{4}^{\prime}$ from (4.38) (which is globally defined and represents an independent 10D degree of freedom). After integration by parts and some cancellation, we see that

$$
S_{\text {eff }}^{5}=\frac{1}{4 \kappa_{10}^{2}}\left\{\int d^{2 \Omega} b_{0}^{I} \wedge \hat{d} \hat{\star} \hat{d} b_{0}^{J} \int \tilde{\star} \omega_{2}^{I} \wedge \gamma_{2}^{J}-\int e^{6 \Omega} e^{4 A} \tilde{d} \tilde{\star} \tilde{d} B_{1}^{Y} \wedge \hat{d} \hat{\star} \hat{d} B_{1}\right\} .
$$

We can simplify this further using the 2-form inner product and the constraint (3.13) to find

$$
\begin{aligned}
S_{\mathrm{eff}}^{5}= & \frac{1}{4 \kappa_{10}^{2}} \int d^{4} x\left[3 \tilde{V} C^{I J} e^{2 \Omega} b_{0}^{I} \hat{\partial}^{2} b_{0}^{J}+\int d^{6} y \sqrt{\tilde{g}} e^{4 \Omega} e^{4 A} \partial^{\tilde{m}} \delta e^{-4 A} \hat{\partial}^{2} B_{m}\right] \\
& -\frac{T_{3}}{2} \int d^{4} x \int d^{6} y \sqrt{\tilde{g}} \tilde{\delta}^{6}(y, Y) e^{4 \Omega} e^{4 A} \delta Y^{\eta h} \Lambda_{\eta p}^{m} \hat{\partial}^{2} B_{m} .
\end{aligned}
$$

The linearized 3-form EOM $\delta E_{8}$ is non-trivial but does not contribute to the quadratic action since $\delta A_{2}$ has the wrong legs to give a non-vanishing wedge product with it.

Finally, the contribution to the action from the brane sector is determined by contracting the dynamical part of the brane EOM with the fluctuation in brane position. As there 
is no WZ term, there is no cancellation between terms involving $g_{\mu m}$ and $C_{4}^{\prime}$. Instead, given that the induced metric is $g_{\mu \nu}$ to linear order, the linearized EOM is

$$
\delta E_{\not h}=\left[e^{-2 \Omega} e^{-4 A} \tilde{g}_{\not h \eta} \hat{\partial}^{2} \delta Y^{\not h}+\hat{\partial}^{2} B_{\not h}-\frac{1}{2} e^{-4 \Omega} e^{-4 A}\left(\hat{\star} \delta E_{6}\right)_{\eta \eta \eta} \sigma^{\not h}\right] \delta^{10}(x, X) .
$$

Therefore, the quadratic action as in (2.4) is

$$
\begin{aligned}
& S_{\text {eff }}^{D 3}=\frac{T_{3}}{2} \int d^{4} x \int d^{6} y \sqrt{\tilde{g}} \tilde{\delta}^{6}(y, Y) \delta Y^{\not h}\left[e^{2 \Omega} \tilde{g}_{\not p h} \hat{\partial}^{2} \delta Y^{\not h}+e^{4 \Omega} e^{4 A} \hat{\partial}^{2} B_{\not h}\right. \\
& \left.-\frac{1}{2} e^{2 \Omega} \hat{\partial}^{2} b_{0}^{I} \gamma_{\eta m h}^{I} \sigma^{\not h}+\frac{1}{2} e^{4 \Omega} \hat{\partial}^{2} \tilde{d}\left(e^{4 A} B_{1}\right)_{\text {mhn }} \sigma^{\not h}\right] \text {. }
\end{aligned}
$$

The second term will cancel with a similar term in $S_{\text {eff }}^{5}$ (4.51). As in section 3.3.2, we have $\tilde{g}_{\not \imath h} \partial_{\mu} Y^{\not h} \partial^{\hat{\mu}} Y^{\natural \hbar}=2 \tilde{g}_{i \bar{\jmath}} \partial_{\mu} Z^{i} \partial^{\hat{\mu}} \bar{Z}^{\bar{\jmath}}$; here and in the following we drop slashes on complex indices for legibility whenever the context is clear.

We are now forced to confront the terms that depend explicitly on the arbitrary reference point through the appearance of $\sigma^{\text {h }}$. We begin with the term proportional to the harmonic (1,1)-form $\gamma_{2}$. In complex coordinates, we can write

$$
\delta Y^{m} \gamma_{m n}^{I} \sigma^{n}=C^{I J}\left(\delta Z^{i} \omega_{i \bar{\jmath}}^{J} \bar{\sigma}^{\bar{\jmath}}+\delta \bar{Z}^{\bar{\jmath}} \omega_{\bar{\jmath} i}^{J} \sigma^{i}\right) .
$$

We know from our earlier calculation in the electric formulation that this term should contain derivatives of the (locally-defined) Kähler potential for the 2 -form defined via $\omega_{i \bar{\jmath}}^{I}=i \partial_{i} \bar{\partial}_{\bar{\jmath}} k^{I}$, so we consider the derivative $\bar{\partial}_{\bar{\jmath}}\left(\omega_{i \bar{k}}^{I} \sigma^{\bar{k}}\right)=\omega_{i \bar{\jmath}}+\left(\bar{\partial}_{\bar{k}} \omega_{i \bar{j}}\right) \sigma^{\bar{k}}$. Because their derivatives are the same, we replace $\omega_{i \bar{\jmath}}^{I} \sigma^{\bar{\jmath}} \rightarrow-i k_{i}^{I}, \omega_{\bar{j} i}^{I} \sigma^{i} \rightarrow i k_{\bar{\jmath}}^{I}$ to lowest order in the formal $\sigma$ expansion. If, instead, our conjectured replacement $\delta_{\perp}^{6}\left(y, Y_{*}\right) \rightarrow \delta_{\perp}^{6}(y, Y)$ is incorrect, we should evaluate this term as $\gamma_{\underline{m n}}^{I} \Lambda \frac{m}{m} \sigma^{\underline{n}}\left(Y_{*}\right)$. Since we can set both $k(Z, \bar{Z})$ and $k_{i}(Z, \bar{Z})$ to zero at $Y_{*}$ by a Kähler transformation, we se that $\gamma_{i \bar{\jmath}}^{I} \Lambda_{\bar{i}}^{\underline{i}} \sigma^{\bar{\jmath}}=C^{I J} \omega_{i \bar{\jmath}}^{J} \Lambda \frac{i}{i} \sigma^{\bar{\jmath}} \sim C^{I J} k_{i}^{J}(Y)$. In fact, this is essentially the approximation used in [1] for these kinetic terms. Presumably, higher order terms in the $\sigma$ expansion would make this approximation exact. In either case, we are led to replace

$$
e^{2 \Omega} \hat{\partial}^{2} b_{0}^{I} \gamma_{\eta \varkappa \eta}^{I} \delta Y^{\eta h} \sigma^{\not h} \rightarrow-i e^{2 \Omega} C^{I J} \hat{\partial}^{2} b_{0}^{I}\left(k_{i}^{J} \delta Z^{i}-k_{\bar{\imath}}^{J} \delta \bar{Z}^{\bar{\imath}}\right)
$$

in precise agreement with (3.47).

The other term, proportional to $\tilde{d}\left(e^{4 A} B_{1}\right)$, is somewhat more subtle because both $e^{-4 A}$ and $B_{1}$ contain Green's functions evaluated at the singular coincidence limit of their arguments. Specifically, $e^{4 A} \rightarrow 0$ at at D3-brane, so the volume modulus and axion compensators do not contribute to (4.53), while the divergence of $B_{1}^{Y}$ can lead a priori to a finite contribution for the D3-brane. ${ }^{17}$ It is possible to show for any 1 -form $v_{1}$ that

$$
\partial_{[\not p}\left(\left(\tilde{d} v_{1}\right)_{\not h] h} \sigma^{\not h}\right)=\left(\tilde{d} v_{1}\right)_{\not p h}-\frac{1}{2} \sigma^{\not h} \tilde{\nabla}_{\not h}\left(\tilde{d} v_{p p h}\right),
$$

\footnotetext{
${ }^{17}$ The compensators $K$ and $B_{1}^{I}$ do in fact solve Poisson equations with singular sources (given by $\tilde{G}(y, Y)$ ), but the subsequent convolution against another Green's function ensures the compensators themselves are smooth.
} 
so it seems reasonable at lowest order in the formal $\sigma$ expansion to replace

$$
\delta Y^{\eta h} e^{4 \Omega} \partial^{\hat{2}} \tilde{d}\left(e^{4 A} B_{1}\right)_{\eta \eta \eta} \sigma^{\not h} \rightarrow \delta Y^{\eta h} e^{4 \Omega} \partial^{\hat{2}} e^{4 A} B_{\eta_{\imath}} \rightarrow-e^{2 \Omega} \delta Y^{\eta h} \partial^{\hat{2}} \delta Y^{\natural \hbar} \lim _{y \rightarrow Y}\left(\Lambda_{m h}^{m} \tilde{g}_{m p} \frac{\tilde{G}_{\not h}^{p}(y, Y)}{\tilde{G}(y, Y)}\right)
$$

in the coincidence limit for the warp factor (3.7) and compensator (3.11). This term has the same general structure as the final term in (3.47), as it provides a not-necessarily-Hermitian contribution to the field space metric for the brane positions. However, the precise form is puzzling. Consistency with the calculation in the electric formalism suggests that we can identify the Green's function form of (4.57) with derivatives of Kähler potentials in combinations proportional to $C^{I J} k_{i}^{I} k_{j}^{J}$, etc. However, while it would be interesting to conjecture that the Green's functions may be related to the Kähler potentials in a similar way, the Green's functions know only about the unwarped CY metric and not the global warp factor or flux information contained in $C^{I J}$. Possibly, higher order terms in the formal $\sigma$ expansion contain this information; we leave it to future work [29] to determine if that is true.

Alternately, we can recall that we assumed that the $\delta$ function in the last term of (4.53) should be evaluated at $Y$ rather than $Y_{*}$. Evaluating it at $Y_{*}$ regulates the Green's functions in $e^{4 A} B_{1}$. Since the effective action cannot depend on the arbitrary point $Y_{*}$, one way to remove the $Y_{*}$ dependence is to average $Y_{*}$ over the CY. This certainly contains global information about the warp factor and flux. Ultimately, a resolution of these issues will require carrying the $\sigma$ expansion to higher (or all) orders. One approach, which we will not pursue here, may be to note that physical quantities like $\tilde{F}_{5}$ are independent of $Y_{*}$, leading to relations between terms at different orders in the $\sigma$ expansion. These relations may help resum the series in a manner similar to renormalization group flow.

Collecting the effective actions from each sector, we can construct the total effective action. Once again, there is significant cancellation between the different sectors. The total remaining action is

$$
\begin{aligned}
S_{\mathrm{eff}}= & -\frac{3 \tilde{V}}{4 \kappa_{10}^{2}} \int d^{4} x\left(e^{4 \Omega} \partial_{\mu} c(x) \partial^{\hat{\mu}} c(x)+e^{2 \Omega} C^{I J} \partial_{\mu} b_{0}^{I} \partial^{\hat{\mu}} b_{0}^{J}\right) \\
& -T_{3} \int d^{4} x\left[e^{2 \Omega} \tilde{g}_{i, \bar{\jmath}}(Y) \partial_{\mu} Z^{i} \partial^{\hat{\mu}} \bar{Z}^{\bar{\jmath}}-\frac{i}{4} C^{I J} \partial_{\mu} b_{0}^{I}\left(k_{\not}^{J} \partial^{\hat{\mu}} Z^{i}-k_{\bar{p}}^{J} \partial^{\hat{\mu}} \bar{Z}^{\bar{\gamma}}\right)\right. \\
& \left.-\frac{1}{2} e^{2 \Omega} \tilde{g}_{m p} \Lambda_{\not h}^{m}\left(\frac{\tilde{G}_{\not h}^{p}(y, Y)}{\tilde{G}(y, Y)}\right) \partial_{\mu} \delta Y^{\eta h} \partial^{\hat{\mu}} \delta Y^{\not h}\right]
\end{aligned}
$$

assuming we use the replacements (4.55), (4.57).

Our final result (4.58) is not manifestly equal to the effective action (3.47) found using the electric formalism for IIB SUGRA, and the two actions may not be equal at all. While a proper treatment must yield the same effective action whether we take electric or magnetic components for $\tilde{F}_{5},(4.58)$ relies on two conjectured replacements $(4.55),(4.57)$ as well as a D3-brane EOM truncated at second order in a formal expansion; we have not yet been able to verify all our assumptions. As a result, it is unsurprising that we do not have precise agreement with the complete derivation of (3.47). However, the agreement of the general 
form of the action is striking: in the magnetic formalism, backreaction of the D3-brane on $\tilde{F}_{5}$ induces a $\partial b_{0} \partial Z$ cross term of the correct form (and correct coefficient, if (4.55) is correct) as well as non-Hermitian $\partial Y \partial Y$ kinetic terms. The possibility that higher orders in the formal $\sigma$ expansion will lead to a relation between the derivatives of the CY Kähler potential and Green's functions is intriguing, but we leave that to the future.

\subsubsection{Summary}

We provided a unified ansatz for fluctuations of the volume modulus, $C_{4}$ axions, and D3-brane position in the magnetic formalism and performed a dimensional reduction on this ansatz. Interestingly, the D3-brane EOM as modified according to the results of the previous subsection contains terms of the form $i \partial b k_{i} \partial Z^{I}+$ c.c. and additional $\partial Y \partial Y$ kinetic terms. In the electric formalism, these terms had appeared due to the WZ action, which does not exist in the magnetic formalism. Although we have not been able to give a full interpretation of these terms in the magnetic formalism - there are possibly more contributions from higher orders in the formal $\sigma$ expansion - it is important to note that these crucial "cross terms" in the kinetic action arise due to the backreaction of the brane motion on the 5-form.

\section{Discussion}

D-branes are important ingredients in flux compactifications, and their dynamics are essential for understanding the structure of the Kähler moduli of the low energy effective theory as well as applications in string phenomenology and cosmology. For instance, several models of inflation in string theory explicitly use the motion of D-branes in warped regions in their construction (see $[7,32]$ ), so a correct description of D-brane dynamics is not of idle interest.

We have shown that a consistent dimensional reduction of $10 \mathrm{D}$ supergravity in the presence of a D3-brane requires the inclusion of fluctuations in the $10 \mathrm{D}$ metric and 5form gauge potential, in addition to the degrees of freedom of the transverse motion of the D3-brane. The D3-brane can couple to the 4-form as either an electric or magnetic source, and we presented for both cases the first consistent set of fluctuations that solve the 10D constraint equations. For a D3-brane coupling as a magnetic source, we find a novel field redefinition of the magnetic 4-form potential that allows the 5-form field strength to be written in terms of a globally-defined 4-form plus delta-function-supported terms that make the dependence of $\tilde{F}_{5}$ on the D3-brane position explicit. The field redefinition leads to additional terms in the D3-brane equation of motion from $\tilde{F}_{5}$, which resolve a puzzle involving the no-force condition on a D3-brane in the magnetic description, as well as contributing important terms to the effective action.

Combining our consistent 10D description of transverse D3-brane degrees of freedom with existing descriptions for the volume modulus [25] and $C_{4}$ axions [26], we performed a careful dimensional reduction to obtain the $4 \mathrm{D}$ effective action. The resulting effective action contains important contributions due to flux and warping, as previously seen in the axion sector in [26]. The calculation involves a remarkable set of cancellations between 
the compensators and Green's functions, demonstrating the importance of a consistent $10 \mathrm{D}$ solution of the constraint equations. We also explicitly demonstrated that there is no "self-energy" problem for the dynamical effective action which might arise from inserting the backreacted brane solution into the brane effective action. The kinetic action includes the expected brane-axion cross-terms as well as kinetic terms for transverse brane motion in addition to the manifest kinetic term in the DBI action. When treating the brane as an electric source, these terms arise from the Hodge dualization of the axions from 2-forms to scalars in the presence of the brane WZ action. When the underlying CY manifold has only a single Kähler modulus, the Kähler potential agrees with the proposal of [7, 15]. In the dimensional reduction treating the brane as a magnetic source, these additional terms arise from the explicit dependence of $\tilde{F}_{5}$ on the brane position along with the backreaction of the brane on the 5 -form. While we have not reproduced the precise form of the additional D3-brane kinetic terms, there are intriguing hints that these terms could be related to Green's functions on the internal space.

Many cancellations in the dimensional reduction likely occur because of the high degree of structure of the background, including supersymmetry and no-scale structure. An effective action for D3-branes in a more general warped background with interesting applications will likely be more complex, and the techniques developed here can play an important role in the necessary calculation. Moving beyond the probe approximation, which we have argued is necessary, it would be interesting to see if there are modifications to the kinetic part of the effective action arising from the interaction of the D3-brane with the 10D fields. As another example, the dynamics of $\overline{\mathrm{D} 3}$-branes in warped flux backgrounds [33] beyond the probe approximation should also include perturbations to the 10D fields in the effective description. We leave a detailed investigation of these and other applications to future work. Nevertheless, we have seen the importance of a consistent 10D description, solving the constraint equations, for constructing a $4 \mathrm{D}$ effective action from dimensional reduction.

\section{Acknowledgments}

We would like to thank L. McAllister for helpful discussions during the early stages of this project and D. Andriot for comments on an earlier version of the manuscript. B. U. would like to acknowledge support by NSERC, an Institute of Particle Physics Postdoctoral Fellowship, and a Lorne Trottier Fellowship from McGill University for support during the early stages of this project. The work of A. F. and B. C. has also been supported by NSERC.

\section{A Conventions}

Here we summarize our conventions and notational shorthand. External, noncompact spacetime coordinates denoted $x^{\mu}$, while internal, compact dimension coordinates are $y^{m}$; when used, $x^{M}$ include all coordinates. Brane worldvolume coordinates are $\xi^{a}$, and the embedding of the worldvolume into spacetime is denoted $X^{M /}(\xi)$ (with $\xi$ dependence sometimes suppressed). The slashed index indicates that the coordinate transforms under dif- 
feomorphisms as the position of the brane as opposed to the spacetime point $x^{M}$ where SUGRA fields are evaluated (see appendix B on bitensors).

Quantities with a hat ${ }^{\wedge}$ are associated with the $4 \mathrm{D}$ metric $\hat{\eta}_{\mu \nu}$, such as raised or lowered indices, the antisymmetric tensor $\hat{\epsilon}_{\mu \nu \lambda \rho}$ (or volume form $\hat{\epsilon}$ ). Similarly, any quantity with a tilde $\sim$ is associated with the unwarped CY metric $\tilde{g}_{m n}$. However, as partial derivatives are metric-independent, we do not accent them (ie, we write $\partial_{\mu}, \partial_{m}$ ) except for raised indices. However, for appearance, we accent the derivatives rather than the square in Laplacians/d'Alembertians, writing $\hat{\partial}^{2}$ and $\tilde{\nabla}^{2}$ rather than $\partial^{\hat{2}}$ and $\tilde{\nabla}^{\tilde{2}}$. Ten-dimensional quantities have capital indices but no accents. A superscript (0) with parentheses indicates a background value.

Two other metrics appear in this paper, the worldvolume metric $\gamma_{a b}$ and an arbitrary metric $g_{m n}^{\perp}$. Indices $a, b$ denote quantities associated with $\gamma_{a b}$; in static gauge $\nabla_{\mu}^{\gamma}$ is also the covariant derivative associated with the worldvolume metric. Quantities associated with $g_{m n}^{\perp}$ are denoted with a $\perp$ sub- or superscript.

We work with a mostly + metric and define the antisymmetric symbol $\epsilon$ as a tensor. Similarly, delta functions are defined as scalars, so $\epsilon$ and $\delta$ implicitly carry $\sqrt{|g|}$ factors. Combinatorial factors for differential forms are defined as in appendix B of [34]. The wedge symbol in a wedge product may be omitted in in-line mathematics.

Harmonic 2-forms are written in terms of a basis $\left\{\omega_{2}^{I}\right\}$ on the CY manifold, so any harmonic form can be written as $e^{I} \omega_{2}^{I}$ for constant coefficients $e^{I}$. This is not the basis of harmonic forms at a single point; if $h_{1,1}$ is greater than the 2 nd Betti number of $T^{6}$, the $\omega_{2}^{I}$ are not all linearly independent at any given point, only as functions. We orthonormalize the basis with respect to the inner product

$$
\int \omega_{2}^{I} \wedge \tilde{\star} \omega_{2}^{J}=3 \tilde{V} \delta^{I J}
$$

This normalization allows us to choose the Kähler form as $\omega_{2}^{1}=\tilde{J}$, since $\tilde{J}^{3}=6 \tilde{\epsilon}$ and $\tilde{\star} \tilde{J}=\tilde{J}^{2} / 2$. (Strictly speaking, in this paper we only consider 2 -forms with positive parity under the orientifold involution, but similar considerations would apply for those with negative parity.)

\section{B Bitensors, expansions, and Green's functions}

As we have noted previously, any attempt to describe the influence of D-branes on SUGRA fields necessarily involves (at least) two points in spacetime: the position where the SUGRA fields are evaluated and the position of the localized brane source (respectively $y^{m}$ and $Y^{\eta n}$ in static gauge). The SUGRA fields are generally functions of both of these positions. We also must consider both positions when evaluating the 10D EOM, as there are bulk equations evaluated at $y^{m}$ and brane equations involving fields evaluated at $Y^{\text {m}}$. At some points, we also introduce a fixed reference point $Y_{*}^{\underline{m}}$. As diffeomorphisms in general act differently at different points, we use distinct markings to indicate which transformation acts on a given tensor index (ie, unmarked, slashed, or underlined). Here we give a very brief review of the properties of tensor functions of two spacetime points, known as bitensors, following [35]. 
A bitensor is a tensorial function of two points in spacetime, which may have indices marked for either of the two points. As an example, the 5 -form $\tilde{F}_{5}$ has 5 indices associated with the evaluation point $y$ but also depends on the brane position $Y$, ie, $\tilde{F}_{m n p q r}(y, Y)$. A key example for us is the 6-dimensional biscalar Dirac distribution (delta function) $\delta^{6}(y, Y)$ (for some metric $g_{m n}$ ) defined by

$$
\int d^{6} y \sqrt{g(y)} f(y) \delta^{6}(y, Y)=f(Y), \quad \int d^{6} Y \sqrt{g(Y)} f(Y) \delta^{6}(y, Y)=f(y) .
$$

Note that the Dirac distribution integrates as a scalar in both coordinates, so it implicitly includes a factor of $1 / \sqrt{g}$. (In the main text, we will consider Dirac distributions for metrics $\tilde{g}_{m n}$ and $g_{m n}^{\perp}$.)

To understand the coincidence limit of a bitensor as well as the expansion of a tensor around a fixed point, we consider the Synge world function. This is a biscalar function of $y, Y$ defined by

$$
\sigma(y, Y)=\frac{1}{2}\left(\lambda_{1}-\lambda_{0}\right) \int_{\lambda_{0}}^{\lambda_{1}} g_{m n}(z) t^{m} t^{n} d \lambda
$$

where $z^{m}(\lambda)$ describes a geodesic with $z\left(\lambda_{0}\right)=y$ and $z\left(\lambda_{1}\right)=Y$ as depicted in figure 2. For affine parameter $\lambda, t^{m}$ is tangent to the geodesic, and $\sigma(y, Y)$ is half the squared geodesic distance between $y$ and $Y$. The derivatives $\sigma_{m} \equiv \partial_{m} \sigma$ and $\sigma_{m p} \equiv \partial_{p_{h}} \sigma$ are tangent to the geodesic at the respective endpoint and directed outward, as in the figure. It can be shown that $\sigma_{m} \sigma^{m}=\sigma_{\eta p} \sigma^{m}=2 \sigma$. A covariantly constant vector $A^{m}$ can be parallel transported from $y$ to $Y$ along the geodesic via the parallel propagator $\Lambda_{n}^{\not h}$ as $A^{\eta h}(Y)=\Lambda_{n}^{\eta h}(y, Y) A^{n}(y)$, with the corresponding generalization for covariantly constant tensors. At coincidence $y=Y, \Lambda_{n}^{\not h}=\delta_{n}^{\not h}$, so any bitensor (with any distribution of indices) satisfies

$$
\begin{aligned}
& T_{m_{1} \cdots m_{a} h_{1} \cdots h_{b}} p_{1} \cdots p_{j} \phi_{1} \cdots \phi_{k}(y, Y)\left(\Lambda_{n_{1}}^{h_{1}} \cdots\right)\left(\Lambda_{q_{1}}^{q_{1}} \cdots\right) \delta(y, Y) \\
& =T_{m_{1} \cdots m_{a} n_{1} \cdots n_{b}} p_{1} \cdots p_{j} q_{1} \cdots q_{k} \\
& (y, y) \delta(y, Y),
\end{aligned}
$$

etc.

There are several useful identities among the Dirac distribution, parallel propagator, and derivatives of the worldfunction. First, because $\sigma^{m} \propto t^{m}$, the tangent to the geodesic, we have

$$
\sigma_{\not h}=-\Lambda_{\not h}^{m} \sigma_{m} \quad \text { and } \quad \sigma_{n}=-\Lambda_{n}^{\not h} \sigma_{\not b} .
$$

Next, in the coincidence limit, parallel propagators are covariantly constant with respect to either endpoint. Finally, the Dirac distribution and the parallel propagator satisfy the identities

$$
\nabla_{m}\left(\Lambda_{\not h}^{m}(y, Y) \delta(y, Y)\right)=-\partial_{\not h} \delta(y, Y), \nabla_{\not h}\left(\Lambda_{n}^{\not h}(y, Y) \delta(y, Y)\right)=-\partial_{n} \delta(y, Y)
$$

in any dimensionality.

Of course, it is often useful to evaluate a tensor as a series expansion around a fixed reference point, preferably in a manifestly covariant manner. One possible application is to 


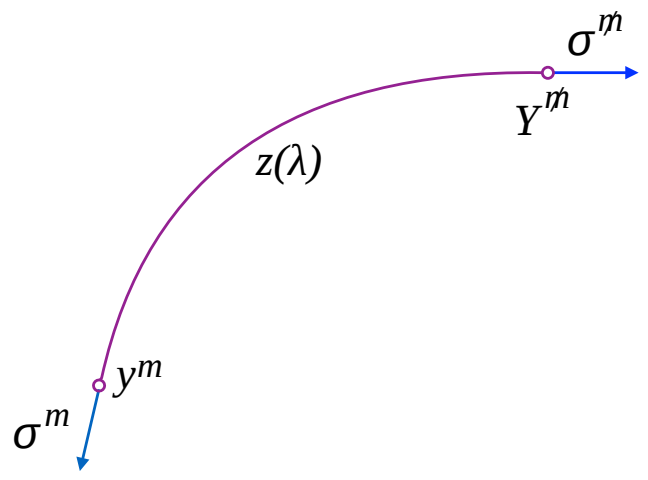

Figure 2. The geodesic $z(\lambda)$ connects $y^{m}$ to $Y^{\eta_{h}} \cdot \sigma^{m}, \sigma^{\text {hh }}$ are outgoing tangents to the geodesic at the endpoints with length equal to the geodesic distance.

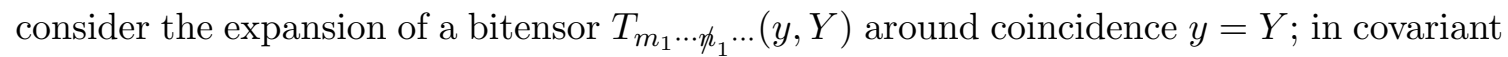
form, this is an expansion in powers of $\sigma^{m}$ (or alternately $\sigma^{\text {th }}$ ) [35]. Another application is the expansion of a tensor as a function of $Y^{\eta h}$ near a reference point $Y_{\star}^{\underline{m}}$. As an example, the expansion of a scalar is

$$
A(Y)=A\left(Y_{*}\right)-\partial_{\underline{n}} A\left(Y_{*}\right) \sigma^{\underline{n}}+\frac{1}{2} \nabla_{\underline{m}} \partial_{\underline{n}} A\left(Y_{*}\right) \sigma^{\underline{m}} \sigma^{\underline{n}}+\cdots,
$$

where the dots represent terms higher order in $\sigma^{\underline{m}}$. In the main text, we use this to expand $\tilde{\delta}^{6}(y, Y)$ around $Y=Y_{\star}$, taking $y$ as a constant, so the delta function is just a scalar function of $Y$.

Finally, let us discuss the behavior of Green's functions on curved space. Consider a minimally coupled massless scalar $\Phi$ and a vector field $A^{m}$ which satisfy the Poisson equations

$$
\nabla^{2} \Phi=-\mu(y), \quad \nabla^{2} A^{m}=-j^{m}(y),
$$

where $\mu, j^{m}$ are sources. We can write the solutions in terms of the biscalar and bitensor Green's functions $G\left(y, y^{\prime}\right)$ and $G_{m^{\prime}}^{m}\left(y, y^{\prime}\right)$ as

$$
\Phi(y)=\int d Y \sqrt{g(Y)} G(y, Y) \mu(Y), \quad A^{m}(y)=\int d Y \sqrt{g(Y)} G_{m p}^{m}(y, Y) j^{p^{h}}(Y)
$$

(in any dimensionality). The Green's functions are defined to satisfy

$$
\nabla^{2} G(y, Y)=-\delta(y, Y), \quad \nabla^{2} G_{m p}^{m}(y, Y)=-\Lambda_{m p}^{m} \delta(y, Y) .
$$

The scalar and tensor Green's functions are related by

$$
\nabla_{m} G_{m p}^{m}(y, Y)=-\partial_{m} G(y, Y)
$$

and vice-versa. We are mostly concerned with the $6 \mathrm{D}$ metric $\tilde{g}_{m n}$ and corresponding Green's functions $\tilde{G}(y, Y)$ and $\tilde{G}_{m}^{m}(y, Y)$. 


\section{Linearized equations of motion}

In this appendix, we assemble all the linearized 10D EOM for the 10D SUGRA fields; these are the Einstein equations, 5-form and 3-form flux equations, and D3-brane position equations. We will identify how the EOM divide into constraints and dynamical EOM; the electric form of the equations are listed first, followed by the magnetic form.

\section{C.1 Electric formalism equations of motion}

Here we provide the EOM for the electric formalism for D3-brane motion. For reference, our ansatz for the SUGRA fields is

$$
\begin{aligned}
& d s^{2}=e^{2 \Omega} e^{2 A} \hat{\eta}_{\mu \nu} d x^{\mu} d x^{\nu}+2 e^{2 \Omega} e^{2 A} \partial_{\mu} B_{m}(x, y) d x^{\mu} d y^{m}+e^{-2 A} \tilde{g}_{m n} d y^{m} d y^{n}, \\
& \tilde{F}_{5}=e^{4 \Omega} \hat{\epsilon} \wedge \tilde{d} e^{4 A}+d\left(e^{4 \Omega} e^{4 A_{\hat{\star}}} \hat{d} B_{1}\right)+\hat{d} b_{2}^{I} \wedge \omega_{2}^{I} \\
& +\left[\tilde{\star} \tilde{d} e^{-4 A}-e^{2 \Omega} e^{-4 A_{\star}} \tilde{\star}\left(\hat{d} B_{1} \wedge \tilde{d} e^{4 A}\right)+\star d\left(e^{4 \Omega} e^{4 A_{\hat{\star}} \hat{d} B_{1}}\right)+e^{-2 \Omega_{\hat{\star}} \hat{d} b_{2}^{I}} \wedge e^{-4 A_{\star}} \tilde{\star} \omega_{2}^{I}\right], \\
& G_{3}=G_{3}^{(0)}-e^{-2 \Omega} \hat{d} \hat{\star} \hat{d} b_{2}^{I} \wedge \Lambda_{1}^{I}+e^{-2 \Omega} \hat{\star} \hat{d} b_{2}^{I} \wedge \tilde{d} \Lambda_{1}^{I},
\end{aligned}
$$

along with $Y^{\eta h}(x)=Y^{(0) \eta h}+\delta Y^{\eta h}(x)$. As in the main text, the components of $\tilde{F}_{5}$ in square brackets are the magnetic components provided for reference as $\star \tilde{F}_{5}$ of the electric components. Above, and throughout, the metric compensator $B_{1}(x, y)$ is the total compensator for all the moduli

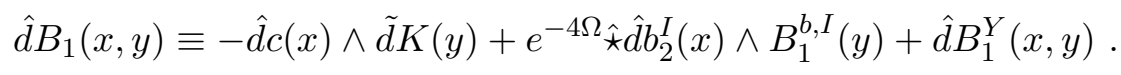

This is clearly an abuse of notation; for reference, $\hat{d} \hat{\star} \hat{d} B_{1}=-\hat{d} \hat{\star} \hat{d} c \tilde{d} K+\hat{d} \hat{\star} \hat{d} B_{1}^{Y}$ and $\hat{d}^{2} B_{1}=$ $e^{-4 \Omega} \hat{d} \hat{\star} \hat{d} b_{2}^{I} B_{1}^{b, I}$ to first order. Furthermore, the warp and Weyl factors include first-order contributions, ie $A=A(x, y)$ and $\Omega=\Omega(x)$. Finally, to allow for the presence of a compensator $\delta C_{4} \sim-\hat{d} b_{2}^{I} K_{1}^{I}$, we do not yet require that $\omega_{2}^{I}$ be harmonic (though it must be closed to avoid terms in $\tilde{F}_{5} \sim b_{2}^{I} \tilde{d} \omega_{2}^{I}$ ). A number of the results in this appendix follow from calculations in [26].

\section{C.1.1 Einstein equations}

The components of the Ricci tensor to first order are

$$
\begin{aligned}
R_{\mu \nu}= & \partial_{\mu} \partial_{\nu}(4 A-2 \Omega)-\hat{\eta}_{\mu \nu} \hat{\partial}^{2}(A+\Omega)+e^{4 A} e^{2 \Omega}\left(\partial^{\tilde{\ell}} A \hat{\partial}^{2} B_{\ell} \hat{\eta}_{\mu \nu}-\tilde{\nabla}^{2} A \hat{\eta}_{\mu \nu}+\partial_{\mu} \partial_{\nu} \tilde{\nabla}^{\tilde{\ell}} B_{\ell}\right), \\
R_{\mu m}= & 2 \partial_{\mu} \partial_{m} A-8 \partial_{\mu} A \partial_{m} A+e^{4 A} e^{2 \Omega}\left(\partial_{\mu} \tilde{\nabla}^{\tilde{\ell}}\left(\tilde{\nabla}_{[m} B_{\ell]}\right)-\tilde{\nabla}^{2} A \partial_{\mu} B_{m}+4 \partial^{\tilde{\ell}} A \partial_{\mu} \tilde{\partial}_{[m} B_{\ell]}\right), \\
R_{m n}= & \hat{\partial}^{2} \tilde{\nabla}_{(m} B_{n)}+4 \partial_{(m} A \hat{\partial}^{2} B_{n)}-\partial^{\tilde{\ell}} A \hat{\partial}^{2} B_{\ell} \tilde{g}_{m n}+\tilde{\nabla}^{2} A \tilde{g}_{m n}+e^{-4 A} e^{-2 \Omega} \hat{\partial}^{2} A \tilde{g}_{m n} \\
& -8 \partial_{m} A \partial_{n} A+\tilde{R}_{m n} .
\end{aligned}
$$

We note for later that we did not need to use $\hat{d}^{2} B_{1}=0$, which is not true off shell for (3.17). Here, $\tilde{R}_{m n}$ is the Ricci tensor of $\tilde{g}_{m n}$; for the CY metrics we consider here, it vanishes, so we set $\tilde{R}_{m n}=0$ henceforth. 
Using these, we can calculate the Ricci curvature, $\mathcal{R}$, to first order

$$
\mathcal{R}=6 e^{-2 A} e^{-2 \Omega} \hat{\partial}^{2}(A-\Omega)+2 e^{2 A}\left(\hat{\partial}^{2} \tilde{\nabla}^{\tilde{\ell}} B_{\ell}+\partial^{\tilde{\ell}} A \hat{\partial}^{2} B_{\ell}+\tilde{\nabla}^{2} A-4 \partial^{\tilde{\ell}} A \partial_{\ell} A\right),
$$

and the Einstein tensor, whose components are

$$
\begin{aligned}
G_{\mu \nu}= & \left(\partial_{\mu} \partial_{\nu}-\hat{\eta}_{\mu \nu} \hat{\partial}^{2}\right)\left(4 A-2 \Omega+e^{4 A} e^{2 \Omega} \tilde{\nabla}^{\tilde{\ell}} B_{\ell}\right)+2 e^{4 A} e^{2 \Omega} \hat{\eta}_{\mu \nu}\left(2 \partial^{\tilde{\ell}} A \partial_{\ell} A-\tilde{\nabla}^{2} A\right), \\
G_{\mu m}= & 2 \partial_{\mu} \partial_{m} A-8 \partial_{\mu} A \partial_{m} A+e^{4 A} e^{2 \Omega} \partial_{\mu}\left(\tilde{\nabla} \tilde{\ell}^{\tilde{\nabla}_{[m}} B_{\ell]}+4 \partial^{\tilde{\ell}} A \tilde{\nabla}_{[m} B_{\ell]}\right. \\
& \left.+2 B_{m}\left(2 \partial^{\ell} A \partial_{\ell} A-\tilde{\nabla}^{2} A\right)\right) \\
G_{m n}= & \hat{\partial}^{2} \tilde{\nabla}_{(m} B_{n)}-\hat{\partial}^{2} \tilde{\nabla}^{\tilde{\ell}_{\ell}} B_{\ell} \tilde{g}_{m n}+4 \partial_{(m} A \hat{\partial}^{2} B_{n)}-2 \partial^{\tilde{\ell}} A \hat{\partial}^{2} B_{\ell} \tilde{g}_{m n}-8 \partial_{m} A \partial_{n} A \\
& +4 \partial^{\tilde{\ell}} A \partial_{\ell} A \tilde{g}_{m n}+e^{-4 A} e^{-2 \Omega} \tilde{g}_{m n} \hat{\partial}^{2}(3 \Omega-2 A) .
\end{aligned}
$$

Next, we determine the stress-energy tensor. We remind the reader that the contributions of the Ramond-Ramond fluxes to the energy-momentum are

$$
T_{M N}^{5}=\frac{1}{4 \cdot 4 !} \tilde{F}_{M P Q R S} \tilde{F}_{N} P Q R S, \quad T_{M N}^{3}=\frac{g_{s}}{4}\left(G_{(M}{ }^{P Q} \bar{G}_{N) P Q}-g_{M N}|G|^{2}\right) .
$$

The resulting energy-momentum tensor for the 5-form (3.19), including terms up to firstorder, has components

$$
\begin{aligned}
T_{\mu \nu}^{5} & =2 e^{4 A} e^{2 \Omega} \hat{\eta}_{\mu \nu}\left(\partial^{\tilde{\ell}} A \hat{\partial}^{2} B_{\ell}-2 \partial^{\tilde{\ell}} A \partial_{\ell} A\right), \\
T_{\mu m}^{5} & =4 e^{4 A} e^{2 \Omega}\left(\partial^{\tilde{\ell}} A \partial_{\mu} \tilde{\nabla}_{[m} B_{\ell]}-\partial^{\tilde{\ell}} A \partial_{\ell} A \partial_{\mu} B_{m}\right)-2 e^{-2 \Omega}\left(\hat{\star} \hat{d} b_{2}^{I}\right)_{\mu} \omega_{m n} \partial^{\tilde{n}} A, \\
T_{m n}^{5} & =4 \partial^{\tilde{\ell}} A \partial_{\ell} A \tilde{g}_{m n}-8 \partial_{m} A \partial_{n} A+4 \partial_{(m} A \hat{\partial}^{2} B_{n)}-2 \partial^{\tilde{\ell}} A \hat{\partial}^{2} B_{\ell} \tilde{g}_{m n} .
\end{aligned}
$$

The resulting energy-momentum tensor for the 3-form (3.20), including terms up to firstorder, has components

$$
\begin{aligned}
T_{\mu \nu}^{3} & =-\frac{g_{s}}{4} e^{2 \Omega} e^{8 A}\left|G_{3}^{(0)}\right|^{\tilde{2}} \hat{\eta}_{\mu \nu}, \\
T_{\mu m}^{3} & =-\frac{g_{s}}{4}\left[i e^{-2 \Omega} e^{4 A}\left(\hat{\star} \hat{d} b_{2}^{I}\right)_{\mu} \tilde{\star}\left(\tilde{d} \Lambda_{1}^{I} \wedge \bar{G}^{(0)}-\text { c.c. }\right)_{m}+e^{2 \Omega} e^{8 A} \partial_{\mu} B_{m}\left|G_{3}^{(0)}\right|^{\tilde{2}}\right] \\
T_{m n}^{3} & =\frac{g_{s}}{4}\left(e^{4 A}\left(G^{(0)}\right)_{m} \tilde{p} \tilde{q}\left(\bar{G}^{(0)}\right)_{n p q}-\tilde{g}_{m n} e^{4 A}\left|G_{3}^{(0)}\right|^{\tilde{2}}\right)=0 .
\end{aligned}
$$

We have used the imaginary self-duality of the background flux, ie $\tilde{\star} G_{3}^{(0)}=i G_{3}^{(0)}$, to simplify (C.12), (C.13).

The energy-momentum tensor for our mobile D3-brane comes from

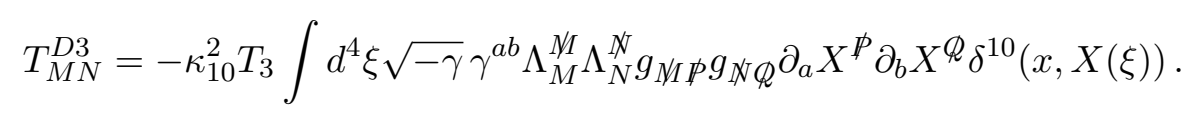

We will use slashed indicies $X^{M}$ to refer to the embedding coordinates of the D3-brane and will work in static gauge $\xi^{a}=\delta_{\mu \mu}^{a} X^{\mu}(\xi)$. The D3-brane energy-momentum tensor, up 
to first order, has components

$$
\begin{aligned}
T_{\mu \nu}^{D 3} & =-\kappa_{10}^{2} T_{3} e^{8 A} e^{2 \Omega} \hat{\eta}_{\mu \nu} \tilde{\delta}^{6}(y, Y), \\
T_{\mu m}^{D 3} & =-\kappa_{10}^{2} T_{3} e^{8 A} e^{2 \Omega} \partial_{\mu} B_{m} \tilde{\delta}^{6}(y, Y)-T_{3} e^{4 A} \tilde{g}_{m \eta} \partial_{\mu} Y^{\not h}(x) \tilde{\delta}^{6}(y, Y), \\
T_{m n}^{D 3} & =0 .
\end{aligned}
$$

Other localized sources (such as other D3-branes and O3-planes) also contribute an energy momentum tensor $T_{M N}^{l o c}$ identical in form to (C.14), (C.15), (C.16), with the exception that $T_{\mu m}^{l o c}$ does not contain the term with explicit dependence on the single mobile brane's position, $\partial_{\mu} Y^{\text {h }}$.

At the end of the day, we obtain the 10D Einstein equations $E_{M N}=G_{M N}-\left(T_{M N}^{5}+\right.$ $T_{M N}^{3}+T_{M N}^{D 3}+T_{M N}^{l o c}$ ) explicitly in terms of our ansatz (through first order in fluctuations):

$$
\begin{aligned}
E_{\mu \nu}= & e^{2 \Omega} e^{4 A}\left[2\left(4 \partial^{\tilde{\ell}} A \partial_{\ell} A-\tilde{\nabla}^{2} A\right)+T_{3} e^{4 A} \tilde{\delta}^{6}(y, Y)+\frac{g_{s}}{4} e^{4 A}\left|G_{3}^{(0)}\right|^{\tilde{2}}+\cdots\right] \hat{\eta}_{\mu \nu} \\
& +\left(\partial_{\mu} \partial_{\nu}-\hat{\eta}_{\mu \nu} \hat{\partial}^{2}\right)\left(4 A-2 \Omega+e^{4 A} e^{2 \Omega} \tilde{\nabla}^{\tilde{\ell}} B_{\ell}\right)-2 e^{4 A} e^{2 \Omega} \hat{\eta}_{\mu \nu} \partial^{\tilde{\ell}} A \hat{\partial}^{2} B_{\ell}, \\
E_{\mu m}= & e^{2 \Omega} e^{4 A} \partial_{\mu} B_{m}\left[2\left(4 \partial^{\tilde{\ell}} A \partial_{\ell} A-\tilde{\nabla}^{2} A\right)+\kappa_{10}^{2} T_{3} e^{4 A} \tilde{\delta}^{6}(y, Y)+\frac{g_{s}}{4} e^{4 A}\left|G_{3}^{(0)}\right|^{\tilde{2}}+\cdots\right] \\
& +2 \partial_{\mu} \partial_{m} A-8 \partial_{\mu} A \partial_{m} A+e^{4 A} e^{2 \Omega} \partial_{\mu} \tilde{\nabla}^{\tilde{\ell}} \tilde{\nabla}_{[m} B_{\ell]}+T_{3} e^{4 A} \tilde{g}_{m \eta} \partial_{\mu} Y^{\hbar h}(x) \tilde{\delta}^{6}(y, Y) \\
& +2 e^{-2 \Omega} e^{4 A}\left(\hat{\star} \hat{d} b_{2}^{I}\right)_{\mu}\left[e^{-4 A} \omega_{m n}^{I} \partial^{\tilde{n}} A+\frac{i g_{s}}{8} \tilde{\star}\left(\tilde{d} \Lambda_{1}^{I} \wedge \bar{G}_{3}^{(0)}-\text { c.c. }\right)\right] \\
E_{m n}= & \hat{\partial}^{2}\left(\tilde{\nabla}_{(m} B_{n)}-\tilde{g}_{m n} \tilde{\nabla}^{\tilde{\ell}} B_{\ell}\right)+e^{-4 A} e^{-2 \Omega} \tilde{g}_{m n} \hat{\partial}^{2}(3 \Omega-2 A),
\end{aligned}
$$

where $\cdots$ denotes the contributions due to local sources other than our mobile D3-brane, whose precise forms are unimportant. The Einstein equations contain both constraints and dynamical EOM For example, the $(\mu \nu)$ and $(\mu m)$ components both contain the Poisson equation (3.21) that determines the warp factor to be (3.23), which now must be satisfied to first order point-by-point on the external spacetime (in this way, our choice of coordinates is similar to the Coulomb gauge of Maxwell theory). The $(\mu \nu)$ component (C.17) also contains a constraint proportional to $\partial_{\mu} \partial_{\nu}-\hat{\eta}_{\mu \nu} \hat{\partial}^{2}$ (yielding (3.22)) and a dynamical EOM in the last term, while the remainder of the $(\mu m)$ component (C.18) is a constraint (3.25) determining the contribution of each modulus to $B_{m}$ (this gives both (3.11), (3.26)). Finally, $E_{m n}$ is entirely second-order in external spacetime derivatives and contributes a dynamical EOM.

\section{C.1.2 Form flux EOM}

In addition to the 10D Einstein equations, we also have 10D EOM from the 5-form and 3 -form fluxes, given by

$$
\begin{aligned}
& E_{6}=d \star \tilde{F}_{5}-\frac{i g_{s}}{2} G_{3} \wedge \bar{G}_{3}+2 \kappa_{10}^{2} T_{3} \int d^{4} \xi \sqrt{-\gamma} \star \epsilon_{\|} \delta^{10}(x, X(\xi)), \\
& E_{8}=d \star G_{3}+i G_{3} \wedge\left(\tilde{F}_{5}+\star \tilde{F}_{5}\right)+\frac{i}{2} A_{2} \wedge E_{6} .
\end{aligned}
$$


Using the ansatz (3.15), (3.19), (3.20), the 5-form EOM becomes

$$
\begin{aligned}
& E_{6}=\tilde{d} \tilde{\star} \tilde{d} e^{-4 A}-\frac{i g_{s}}{2} G_{3}^{(0)} \wedge \bar{G}_{3}^{(0)}-2 \kappa_{10}^{2} T_{3} \tilde{\epsilon} \tilde{\delta}^{6}(y, Y)+\tilde{d}\left(e^{-4 A_{\star}} \hat{\partial}^{2} B_{1}\right) \\
& +\hat{d}\left[\tilde{\star} \tilde{d} e^{-4 A}+e^{2 \Omega} \tilde{d} \tilde{\star} \tilde{d} B_{1}-2 \kappa_{10}^{2} T_{3} \tilde{\star} \tilde{Y}_{1} \tilde{\delta}^{6}(y, Y)+e^{-4 A} \hat{\partial}^{2} \tilde{\star} B_{1}\right]
\end{aligned}
$$

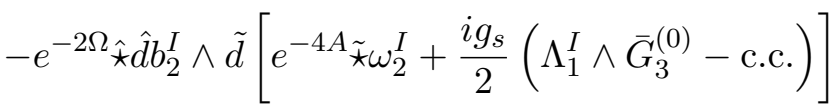

$$
\begin{aligned}
& +e^{-2 \Omega} \hat{d} \hat{\star} \hat{d} b_{2}^{I} \wedge\left[e^{-4 A} \tilde{\star} \omega_{2}^{I}-\tilde{\star} \tilde{d} B_{1}^{b, I}+\frac{i g_{s}}{2}\left(\Lambda_{1}^{I} \wedge \bar{G}_{3}^{(0)}-\text { c.c. }\right)\right] .
\end{aligned}
$$

Each component of $E_{6}$ leads to a distinct constraint or dynamical EOM. The constraints largely repeat those from the Einstein equations: the first three terms of (C.20) are once again the instantaneous Poisson equation for the warp factor. For reasons explained in footnote 9 , we ignore the $\hat{d} \hat{\partial}^{2} \tilde{\star} B_{1}$ term, so the $(1,5)$ components of $E_{6}$ nearly reproduce (C.18) (up to a Hodge star). They differ only by a term proportional to $\tilde{d} \tilde{\star} \omega_{2}^{I}$; therefore, we see that $\omega_{2}^{I}$ must be harmonic or alternately that any compensator $\hat{d} b_{2} K_{1}$ in $C_{4}$ must vanish. The dynamical EOM include $(0,6)$ and $(2,4)$ components and yield $(3.36)$ once the definition (3.28) is taken into account.

Meanwhile, the 3-form EOM becomes

$$
\begin{aligned}
& E_{8}=-\hat{d} b_{2}^{I} \wedge\left[\tilde{d} \tilde{\star} \tilde{d} \Lambda_{1}^{I}+i \omega_{2}^{I} \wedge G_{3}^{(0)}\right]+e^{-2 \Omega} e^{-4 A}\left(\hat{d} \hat{\star} \hat{d} \hat{\star} \hat{d} b_{2}^{I}\right) \wedge \tilde{\star} \Lambda_{1}^{I}
\end{aligned}
$$

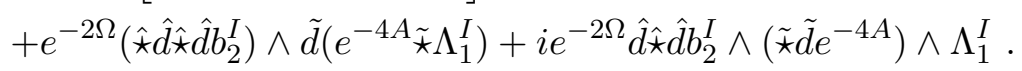

The first term (in square brackets) gives (3.29), the constraint determining $\Lambda_{1}^{I}$. The remainder of the terms give the dynamical EOM $\delta E_{8}$, but do not contribute to the 2-derivative quadratic action because they either have the wrong legs to wedge with $\delta A_{2}$, contribute only at higher derivative order, or both.

\section{C.1.3 Brane EOM}

Finally, the D3-brane EOM is

$$
\begin{aligned}
& E_{M I}=\nabla_{a}\left[\left(g_{M I N} \partial^{a} X^{N_{N}}+\frac{1}{6} \frac{\mu_{3}}{T_{3}} \epsilon^{a b c d} C_{M I N \perp P Q} \partial_{b} X^{\not N} \partial_{c} X^{\not P} \partial_{d} X^{Q}\right) \delta^{10}(x, X)\right]
\end{aligned}
$$

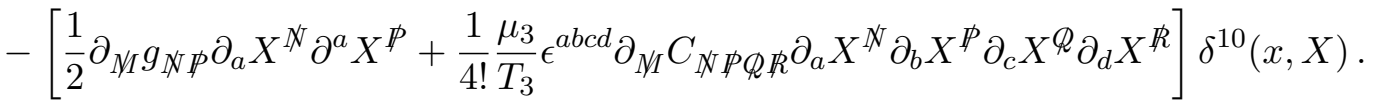

As noted in the main text, we have ignored terms proportional to the derivative of the delta function, as they vanish in the variation of the action upon integration by parts.

We evaluate these EOM in the static gauge using the 4-form background corresponding to $(3.19)$, ie

$$
C_{4}=e^{4 \Omega} e^{4 A} \hat{\epsilon}+e^{4 \Omega} e^{4 A_{\hat{\star}} \hat{d} B_{1}}+b_{2}^{I} \wedge \omega_{2}^{I} .
$$

Then the $M=\mu$ component of the D3-brane EOM becomes (again, dropping terms proportional to the derivative of the delta function)

$$
E_{\not \mu}=-\left[2 e^{-2 A-2 \Omega} \partial_{\not \mu}\left(e^{2 A+2 \Omega}\right)-e^{-4 A-4 \Omega} \partial_{\not \mu}\left(e^{4 A+4 \Omega}\right)\right] \delta^{10}(x, X)=0,
$$


which is a trivial constraint. The $M=\not h$ component gives a dynamical EOM

$$
E_{\not h}=e^{-4 A} e^{-2 \Omega} \tilde{g}_{\eta \eta n}\left(\hat{\partial}^{2} Y^{\not h}\right) \delta^{10}(x, X) .
$$

The second line of (2.14) vanishes identically, and the terms in the first line containing $g_{m \mu t}$ and $C_{\eta \mu \mu \psi \phi}$, both of which include the $B_{1}$ compensator, cancel each other.

\section{C.2 Magnetic equations of motion}

In this section, we will compute the 10D EOM for the gravity, 5-form flux, 3-form flux, and local sources for the magnetic form. The ansatz for all moduli is

$$
\begin{aligned}
& d s^{2}=e^{2 \Omega} e^{2 A} \hat{\eta}_{\mu \nu} d x^{\mu} d x^{\nu}+2 e^{2 \Omega} e^{2 A} \partial_{\mu} B_{m}(x, y) d x^{\mu} d y^{m}+e^{-2 A} \tilde{g}_{m n} d y^{m} d y^{n}, \\
& \tilde{F}_{5}=\tilde{\star} \tilde{d} e^{-4 A}-e^{2 \Omega} \hat{d}\left(\tilde{\star} \tilde{d} B_{1}^{Y}\right)+\hat{d} b_{0}^{I} \wedge\left(\tilde{\star} \omega_{2}^{I}+\tilde{d} K_{3}^{I}-\frac{i g_{s}}{2}\left(\Lambda_{1}^{I} \wedge \bar{G}_{3}^{(0)}-\bar{\Lambda}_{1}^{I} \wedge G_{3}^{(0)}\right)\right) \\
& +\left[e^{4 \Omega} \hat{\epsilon} \wedge \tilde{d} e^{4 A}-e^{4 \Omega_{\hat{\star}} \hat{d} \tilde{d}}\left(e^{4 A} B_{1}\right)+e^{2 \Omega_{\hat{\star}}} \hat{d} b_{0}^{I} \wedge \gamma_{2}^{I}\right], \\
& G_{3}=G_{3}^{(0)}+\hat{d} b_{0}^{I} \wedge \tilde{d} \Lambda_{1}^{I},
\end{aligned}
$$

plus $Y^{\not h}(x)=Y^{(0) \eta h}+\delta Y^{\eta h}(x)$. In the main text, we also consider the relation of $Y^{\eta^{h}}(x)$ to reference point $Y_{*}^{\underline{m}}$; they are connected by a geodesic which has outward-pointing tangents $\sigma^{\underline{m}}, \sigma^{\underline{h}}$ at the endpoints. The metric compensator and (redefined) 4 -form perturbation are

$$
\begin{aligned}
B_{1} & =-c(x) \tilde{d} K(y)+b_{0}^{I}(x) B_{1}^{I}(y)+B_{1}^{Y}(x, y), \\
\delta C_{4}^{\prime} & =b_{0}^{I}(x) \tilde{\star} \omega_{2}^{I}(y)-\hat{d} b_{0}^{I} K_{3}^{I}(y)-e^{2 \Omega} \tilde{\star} \tilde{d} B_{1}^{Y}(x, y) .
\end{aligned}
$$

As before, $\Omega(x), A(x, y)$ contain both background and first-order parts. Here, $\omega_{2}^{I}$ is harmonic, and there is an explicit compensator $K_{3}^{I}$ for the axions. The form $\gamma_{2}^{I}$ is shorthand for

$$
\gamma_{2}^{I} \equiv e^{4 A}\left[\omega_{2}^{I}+\tilde{\star}\left(\tilde{d} K_{3}^{I}-\frac{i g_{s}}{2}\left(\tilde{d} \Lambda_{1}^{I} \wedge \bar{G}_{3}^{(0)}-\text { c.c. }\right)\right)+e^{2 \Omega} \tilde{d} B_{1}^{I}\right] \equiv C^{I J} \omega_{2}^{J},
$$

which we will motivate from the constraints below; as we will also see that $\gamma_{2}^{I}$ must be harmonic, $C^{I J}$ is a change-of-basis matrix defined as in (3.38), which can depend on the background values of the moduli in general. Again, many of the following results are adapted from [26].

\section{C.2.1 Einstein equations}

The metric is the same in the magnetic formalism as the electric formalism, with the exception that the $B_{m}$ compensator takes a somewhat different form. However, the Einstein tensor is independent of the particular form of $B_{m}$, so the Einstein tensor is still given by equations (C.5), (C.6), (C.7).

The energy-momentum tensors are similar to the electric formalism but not quite identical. We find

$$
\begin{aligned}
& T_{\mu \nu}^{5}=-4 e^{2 \Omega} e^{4 A}\left(\partial^{\tilde{\ell}} A \partial_{\ell} A\right) \hat{\eta}_{\mu \nu} \\
& T_{\mu m}^{5}=-4 e^{2 \Omega} e^{4 A}\left(\partial^{\tilde{\ell}} A \partial_{\ell} A\right) \partial_{\mu} B_{m}+2 e^{2 \Omega} e^{4 A} \partial_{\mu}(\tilde{d} B)_{m n} \partial^{\tilde{n}} A-2 \partial_{\mu} b_{0}^{I} \gamma_{m n}^{I} \partial^{\tilde{n}} A, \\
& T_{m n}^{5}=4 \partial^{\tilde{\ell}} A \partial_{\ell} A \tilde{g}_{m n}-8 \partial_{m} A \partial_{n} A
\end{aligned}
$$


for the 5-form contribution based on [26]. Since the only compensator in $G_{3}$ is for the axions, its contribution to the energy-momentum tensor is identical to [26]:

$$
\begin{aligned}
T_{\mu \nu}^{3} & =-\frac{g_{s}}{4} e^{2 \Omega} e^{8 A}\left|G_{3}^{(0)}\right|^{\tilde{2}} \hat{\eta}_{\mu \nu}, \\
T_{\mu m}^{3} & =-\frac{g_{s}}{4}\left[-i e^{4 A} \partial_{\mu} b_{0}^{I} \tilde{\star}\left(\tilde{d} \Lambda_{1}^{I} \wedge \bar{G}_{3}^{(0)}-\text { c.c. }\right)_{m}+e^{2 \Omega} e^{8 A} \partial_{\mu} B_{m}\left|G_{3}^{(0)}\right|^{\tilde{2}}\right], \\
T_{m n}^{3} & =0 .
\end{aligned}
$$

In static gauge, the energy-momentum tensor for the D3-brane is still given by (C.14), (C.15), (C.16) since it is unaffected by the ansatz for the flux.

In the end, the Einstein equations through first order are

$$
\begin{aligned}
E_{\mu \nu}= & e^{2 \Omega} e^{4 A}\left[2\left(4 \partial^{\tilde{\ell}} A \partial_{\ell} A-\tilde{\nabla}^{2} A\right)+T_{3} e^{4 A} \tilde{\delta}^{6}(y, Y)+\frac{g_{s}}{4} e^{4 A}\left|G_{3}^{(0)}\right|^{\tilde{2}}+\cdots\right] \hat{\eta}_{\mu \nu} \\
& +\left(\partial_{\mu} \partial_{\nu}-\hat{\eta}_{\mu \nu} \hat{\partial}^{2}\right)\left(4 A-2 \Omega+e^{4 A} e^{2 \Omega} \tilde{\nabla}^{\tilde{\ell}} B_{\ell}\right), \\
E_{\mu m}= & e^{2 \Omega} e^{4 A} \partial_{\mu} B_{m}\left[2\left(4 \partial^{\ell} A \partial_{\ell} A-\tilde{\nabla}^{2} A\right)+\kappa_{10}^{2} T_{3} e^{4 A} \tilde{\delta}^{6}(y, Y)+\frac{g_{s}}{4} e^{4 A}\left|G_{3}^{(0)}\right|^{\tilde{2}}+\cdots\right] \\
& +2 \partial_{\mu} \partial_{m} A-8 \partial_{\mu} A \partial_{m} A+e^{4 A} e^{2 \Omega} \partial_{\mu} \tilde{\nabla}^{\tilde{\ell}} \tilde{\nabla}_{[m} B_{\ell]}+T_{3} e^{4 A} \tilde{g}_{m x} \partial_{\mu} Y^{\not h}(x) \tilde{\delta}^{6}(y, Y) \\
& +2 e^{4 A} \partial_{\mu} b_{0}^{I}\left[e^{-4 A} \gamma_{m n}^{I} \partial^{\tilde{n}} A+\frac{i g_{s}}{8} \tilde{\star}\left(\tilde{d} \Lambda_{1}^{I} \wedge \bar{G}_{3}^{(0)}-\text { c.c. }\right)\right], \\
E_{m n}= & \hat{\partial}^{2}\left(\tilde{\nabla}_{(m} B_{n)}+\partial_{(m} A B_{n)}-\tilde{\nabla}^{\tilde{\ell}} B_{\ell} \tilde{g}_{m n}-2 \tilde{g}_{m n} \tilde{\nabla}^{\tilde{\ell}} A B_{\ell}\right)+e^{-4 A} e^{-2 \Omega} \tilde{g}_{m n} \hat{\partial}^{2}(3 \Omega-2 A) .
\end{aligned}
$$

As before, (C.30) contains the instantaneous version of the Poisson equation determining the warp factor, which yields (3.23), along with the constraint (3.22) for $\tilde{\nabla}^{\tilde{n}} B_{n}$ (including (3.24) for $K$, the volume modulus compensator). The off-diagonal Einstein equation (C.31) also includes the Poisson equation for the warp factor, along with a Poisson equation (3.10) for $B_{m}^{Y}$ (which is satisfied by (3.11)), and the Poisson equation

$$
\tilde{\nabla}^{2} B_{m}^{I}=-e^{-2 \Omega} \gamma_{m n}^{I} \partial^{\tilde{n}} e^{-4 A}-\frac{i g_{s}}{2} \tilde{\star}\left(\tilde{d} \Lambda_{1}^{I} \wedge \bar{G}_{3}^{(0)}-\text { c.c. }\right)_{m} .
$$

The internal component (C.19) is once again a dynamical EOM only, and we can simplify it to the form (4.44) using (3.22).

\section{C.2.2 Form flux EOM}

Because the magnetic ansatz (4.39) for $\tilde{F}_{5}$ differs considerably from the electric case, the EOM for $\tilde{F}_{5}$ and $G_{3}$ also differ significantly from the electric formalism.

The first thing to note is that neither the 3-form or D3-brane source terms have the correct components to contribute to $E_{6}$ in the magnetic formalism; $E_{6}=d \star \tilde{F}_{5}$, as explained in section 2.3. Therefore, the EOM becomes

$$
E_{6}=-e^{4 \Omega} \hat{d} \hat{\star} \hat{d} \tilde{d}\left(e^{4 A} B_{1}\right)+e^{2 \Omega} \hat{d} \hat{\star} \hat{d} b_{0}^{I} \wedge \gamma_{2}^{I}+e^{2 \Omega} \hat{\star} \hat{d} b_{0}^{I} \wedge \tilde{d} \gamma_{2}^{I}
$$


The first two terms contribute to the dynamical EOM, while the last term is a constraint requiring that $\gamma_{2}^{I}$, defined in terms of the moduli and compensators as (4.42), be closed. Meanwhile, the Bianchi identity is now the constraint

$$
d \tilde{F}_{5}-\frac{i g_{s}}{2} G_{3} \wedge \bar{G}_{3}+2 \kappa_{10}^{2} T_{3} \int d^{4} \xi \sqrt{-\gamma} \star \epsilon_{\|} \delta^{10}(x, X(\xi))=0 .
$$

Written as in (4.39), $\tilde{F}_{5}$ automatically satisfies the Bianchi identity as long as $B_{1}^{Y}$ is given by (3.11). However, in terms of the shorthand variable $\gamma_{2}^{I}$, the magnetic components of $\tilde{F}_{5}$ are $\tilde{F}_{5}=\tilde{d} e^{-4 A}-e^{2 \Omega} \hat{d}\left(\tilde{\star} \tilde{d} B_{1}\right)+e^{2 \Omega} e^{-4 A} \hat{d} b_{0}^{I} \tilde{\star} \gamma_{2}^{I}$. Like the $(\mu m)$ component of the Einstein equation, the constraint from the Bianchi identity in these variables leads to (3.10) for $B_{1}^{Y}$ and (C.33) if and only if $\tilde{d} \tilde{\star} \gamma_{2}^{I}=0$, which implies that $\gamma_{2}^{I}$ is harmonic.

The 3 -form EOM is

$$
E_{8}=e^{2 \Omega} \hat{d} \hat{\star} \hat{d} b_{0}^{I} \wedge \tilde{\star} \tilde{d} \Lambda_{1}^{I}-e^{2 \Omega_{\hat{\star}}} \hat{d} b_{0}^{I} \wedge \tilde{d} \tilde{\star} \tilde{d} \Lambda_{1}^{I}+i e^{4 \Omega} e^{4 A} \hat{d} \hat{\star} \hat{d} B_{1} \wedge G_{3}^{(0)}-i e^{2 \Omega_{\hat{\star}}} \hat{d} b_{0}^{I} \wedge \gamma_{2}^{I} \wedge G_{3}^{(0)} .
$$

The first and third terms contribute to the dynamical EOM, while the second and fourth, when acted on by $\tilde{\star}$, give the Poisson equation (4.41) for $\Lambda_{1}^{I}$.

\section{C.2.3 Brane EOM}

As noted in the main text, the D3-brane action has no WZ term through second order in $\hat{d} Y$ in the magnetic formalism because $C_{4}$ has the wrong legs. However, as we discussed extensively, we should properly think of $\tilde{F}_{5}$ as depending explicitly on the brane position because of the nontrivial Bianchi identity, much as it depends on $A_{2}$ and $G_{3}$. As a result, the $\tilde{F}_{5}$ kinetic action contributes to the D3-brane EOM, which we derived in section 4.2.3. With some conjectures about higher-order terms in a formal expansion, the EOM in static gauge comes out to

$$
\begin{aligned}
& E_{\not h}=\left\{\nabla_{\mu}^{\gamma}\left(\gamma^{\mu \nu} g_{\not h \eta} \partial_{\nu} Y^{\not h}+\gamma^{\mu \nu} g_{\nu \not h}\right)-\frac{1}{2} \gamma^{\mu \nu}\left(\partial_{\not h} g_{\mu \nu}+2 \partial_{\not h} g_{\not h(\mu} \partial_{\nu)} Y^{\not h}+\partial_{\not h} g_{\not h p} \partial_{\mu} Y^{\not h} \partial_{\nu} Y^{\not p}\right)\right. \\
& \left.-\frac{1}{2}\left(\star_{\gamma} d \star \tilde{F}_{5}\right)_{n \eta \eta} \Lambda_{\underline{n}}^{n} \sigma^{\underline{n}}+\frac{\sqrt{-g}}{\sqrt{-\gamma} \sqrt{g_{\perp}}} \frac{1}{5 !}\left(\left(\epsilon_{\perp}\right)_{\eta n n p q r s} \tilde{F}^{n p q r s}+\left(\epsilon_{\perp}\right)_{\eta h \eta p q r s} \tilde{F}^{\mu p q r s} \partial_{\mu} Y^{\not h}\right)\right\} \\
& \times \delta^{10}(x, X) \text {. }
\end{aligned}
$$

We recall that $\gamma_{\mu \nu}$ is the (independent) worldvolume metric, $g_{\perp, m n}$ is an arbitrary metric on the $y^{m}$ coordinates, and $\sigma^{\underline{m}}$ is the tangent to a geodesic from the brane position $Y^{m p}$ to an arbitrary reference point $Y_{*}^{\underline{m}}$ at $Y_{*}^{\underline{m}}$. As usual, the EOM for $\gamma_{\mu \nu}$ sets it equal to the pullback of $g_{M N}$ to the brane worldvolume, but $\gamma_{\mu \nu}$ has no first-order fluctuation. For convenience, we take $g_{\perp, m n}=\tilde{g}_{m n}$.

In (4.31), the background terms involving $\partial_{\eta \downarrow} g_{\mu \nu}$ and $\left(\epsilon_{\perp} \cdot \tilde{F}_{5}\right)_{\not h}$ cancel each other; this is the no-force condition on the D3-brane in the magnetic formalism. A number of terms enter at second order, leaving a first-order dynamical EOM of

$$
\delta E_{\not h}=\left[e^{-2 \Omega} e^{-4 A} \tilde{g}_{\eta \eta \not h} \hat{\partial}^{2} \delta Y^{\not h}+\hat{\partial}^{2} B_{\not h}-\frac{1}{2} e^{-4 \Omega} e^{-4 A}\left(\hat{\star} \delta E_{6}\right)_{\eta \not h} \sigma^{\not h}\right] \delta^{10}(x, X) .
$$




\section{Kähler potential and kinetic action}

As is well-known, GKP compactifications have $\mathcal{N}=1$ SUGRA (possibly with spontaneously broken supersymmetry) as their $4 \mathrm{D}$ effective theory. As a result, the metric on moduli space must be Kähler, meaning that moduli space is complex and that the hermitean metric on moduli space is Kähler $G_{a \bar{b}}=\partial_{a} \partial_{\bar{b}} \mathcal{K}$. Here we consider the kinetic Lagrangian $\mathcal{L}=G_{a \bar{b}} \partial_{\mu} \phi^{a} \partial^{\mu} \bar{\phi}^{\bar{b}}$ for the Kähler potential

$$
\mathcal{K}=-3 \log [-i(\rho-\bar{\rho})-\gamma k(Z, \bar{Z})]
$$

which is appropriate for the case that $h^{1,1}=1$. In relation to the variables of the $10 \mathrm{D}$ SUGRA fields, $\rho=b+i(c+\gamma k(Z, \bar{Z}) / 2)$, and $k(Z, \bar{Z})$ is the Kähler potential of the underlying CY manifold.

The kinetic Lagrangian for these moduli takes the form

$$
\mathcal{L}=\partial_{\rho} \partial_{\bar{\rho}} \mathcal{K} \partial \rho \partial \bar{\rho}+\partial_{\rho} \partial_{\bar{Z}} \mathcal{K} \partial \rho \partial \bar{Z}+\partial_{\bar{\rho}} \partial_{Z} \mathcal{K} \partial \bar{\rho} \partial Z+\partial_{Z} \partial_{\bar{Z}} \mathcal{K} \partial Z \partial \bar{Z}
$$

Using (D.1), each term is:

$$
\begin{aligned}
\partial_{\rho} \partial_{\bar{\rho}} \mathcal{K} \partial \rho \partial \bar{\rho}= & \frac{3}{4 c^{2}}\left((\partial b)^{2}+(\partial c)^{2}+\gamma \partial c\left(\partial_{Z} k \partial Z+\partial_{\bar{Z}} k \partial \bar{Z}\right)\right. \\
& \left.+\frac{\gamma^{2}}{4}\left(\partial_{Z} k \partial Z+\partial_{\bar{Z}} k \partial \bar{Z}\right)^{2}\right) \\
\partial_{\rho} \partial_{\bar{Z}} \mathcal{K} \partial \rho \partial \bar{Z}= & i \frac{3 \gamma \partial_{\bar{Z}} k}{4 c^{2}}\left(\partial b+i \partial c+i \frac{\gamma}{2}\left(\partial_{Z} k \partial Z+\partial_{\bar{Z}} k \partial \bar{Z}\right)\right) \partial \bar{Z}, \\
\partial_{\bar{\rho}} \partial_{Z} \mathcal{K} \partial \bar{\rho} \partial Z= & -i \frac{3 \gamma \partial_{Z} k}{4 c^{2}}\left(\partial b-i \partial c-i \frac{\gamma}{2}\left(\partial_{\bar{Z}} k \partial \bar{Z}+\partial_{Z} k \partial Z\right)\right) \partial Z, \\
\partial_{Z} \partial_{\bar{Z}} \mathcal{K} \partial Z \partial \bar{Z}= & \frac{3 \gamma}{2 c}\left(\partial_{Z} \partial_{\bar{Z}} k+\frac{\gamma \partial_{Z} k \partial_{\bar{Z}} k}{2 c}\right) \partial Z \partial \bar{Z} .
\end{aligned}
$$

Adding everything together gives

$$
\begin{aligned}
\mathcal{L}= & \frac{3}{4 c^{2}}\left((\partial b)^{2}+(\partial c)^{2}\right)+\frac{3 \gamma}{2 c} \partial_{Z} \partial_{\bar{Z}} k \partial Z \partial \bar{Z}-\frac{3 i \gamma}{4 c^{2}} \partial b\left(\partial_{Z} k \partial Z-\partial_{\bar{Z}} k \partial \bar{Z}\right) \\
& -\frac{3 \gamma^{2}}{16 c^{2}}\left(\partial_{Z} k \partial_{Z} k \partial Z \partial Z-2 \partial_{Z} k \partial_{\bar{Z}} k \partial Z \partial \bar{Z}+\partial_{\bar{Z}} k \partial_{\bar{Z}} k \partial \bar{Z} \partial \bar{Z}\right) .
\end{aligned}
$$

Based on the form of (D.7), what we anticipate seeing in the quadratic action are separate quadratic terms for the scalar axion, volume modulus, and the D3-brane; a second-derivative of the internal Kähler potential; sets of derivatives (holomorphic, antiholomorphic, and mixed) acting on $k$; and a coupling between the axion and D3-brane moduli.

Open Access. This article is distributed under the terms of the Creative Commons Attribution License (CC-BY 4.0), which permits any use, distribution and reproduction in any medium, provided the original author(s) and source are credited. 


\section{References}

[1] M. Graña, T.W. Grimm, H. Jockers and J. Louis, Soft supersymmetry breaking in Calabi-Yau orientifolds with D-branes and fluxes, Nucl. Phys. B 690 (2004) 21 [hep-th/0312232] [INSPIRE].

[2] T.W. Grimm and J. Louis, The effective action of $N=1$ Calabi-Yau orientifolds, Nucl. Phys. B 699 (2004) 387 [hep-th/0403067] [INSPIRE].

[3] T.W. Grimm and J. Louis, The effective action of type IIA Calabi-Yau orientifolds, Nucl. Phys. B 718 (2005) 153 [hep-th/0412277] [INSPIRE].

[4] M. Graña, Flux compactifications in string theory: a comprehensive review, Phys. Rept. 423 (2006) 91 [hep-th/0509003] [INSPIRE].

[5] A. Maharana and E. Palti, Models of Particle Physics from Type IIB String Theory and F-theory: A Review, Int. J. Mod. Phys. A 28 (2013) 1330005 [arXiv:1212.0555] [InSPIRE].

[6] S. Kachru, R. Kallosh, A.D. Linde and S.P. Trivedi, de Sitter vacua in string theory, Phys. Rev. D 68 (2003) 046005 [hep-th/0301240] [INSPIRE].

[7] S. Kachru, R. Kallosh, A.D. Linde, J.M. Maldacena, L.P. McAllister and S.P. Trivedi, Towards inflation in string theory, JCAP 10 (2003) 013 [hep-th/0308055] [INSPIRE].

[8] C.P. Burgess, M. Cicoli and F. Quevedo, String Inflation After Planck 2013, JCAP 11 (2013) 003 [arXiv: 1306.3512] [INSPIRE].

[9] D. Baumann and L. McAllister, Inflation and String Theory, arXiv:1404.2601.

[10] V.F. Mukhanov, H.A. Feldman and R.H. Brandenberger, Theory of cosmological perturbations. Part 1. Classical perturbations. Part 2. Quantum theory of perturbations. Part 3. Extensions, Phys. Rept. 215 (1992) 203 [INSPIRE].

[11] K. Becker and M. Becker, M theory on eight manifolds, Nucl. Phys. B 477 (1996) 155 [hep-th/9605053] [INSPIRE].

[12] K. Dasgupta, G. Rajesh and S. Sethi, M theory, orientifolds and G-flux, JHEP 08 (1999) 023 [hep-th/9908088] [INSPIRE].

[13] B.R. Greene, K. Schalm and G. Shiu, Warped compactifications in $M$ and $F$ theory, Nucl. Phys. B 584 (2000) 480 [hep-th/0004103] [INSPIRE].

[14] S.B. Giddings, S. Kachru and J. Polchinski, Hierarchies from fluxes in string compactifications, Phys. Rev. D 66 (2002) 106006 [hep-th/0105097] [InSPIRE].

[15] O. DeWolfe and S.B. Giddings, Scales and hierarchies in warped compactifications and brane worlds, Phys. Rev. D 67 (2003) 066008 [hep-th/0208123] [INSPIRE].

[16] P. Koerber and L. Martucci, From ten to four and back again: How to generalize the geometry, JHEP 08 (2007) 059 [arXiv:0707.1038] [INSPIRE].

[17] L. Martucci, On moduli and effective theory of $N=1$ warped flux compactifications, JHEP 05 (2009) 027 [arXiv:0902.4031] [InSPIRE].

[18] L. Martucci, Warping the Kähler potential of F-theory/IIB flux compactifications, JHEP 03 (2015) 067 [arXiv:1411.2623] [INSPIRE].

[19] L. Martucci, Warped Kähler potentials and fluxes, arXiv:1610.02403 [INSPIRE].

[20] B. Underwood, A Breathing Mode for Warped Compactifications, Class. Quant. Grav. 28 (2011) 195013 [arXiv: 1009.4200] [INSPIRE]. 
[21] A.R. Frey and J. Polchinski, $N=3$ warped compactifications, Phys. Rev. D 65 (2002) 126009 [hep-th/0201029] [INSPIRE].

[22] S.B. Giddings and A. Maharana, Dynamics of warped compactifications and the shape of the warped landscape, Phys. Rev. D 73 (2006) 126003 [hep-th/0507158] [INSPIRE].

[23] M.R. Douglas and G. Torroba, Kinetic terms in warped compactifications, JHEP 05 (2009) 013 [arXiv: 0805.3700] [INSPIRE].

[24] G. Shiu, G. Torroba, B. Underwood and M.R. Douglas, Dynamics of Warped Flux Compactifications, JHEP 06 (2008) 024 [arXiv:0803.3068] [INSPIRE].

[25] A.R. Frey, G. Torroba, B. Underwood and M.R. Douglas, The Universal Kähler Modulus in Warped Compactifications, JHEP 01 (2009) 036 [arXiv: 0810.5768] [INSPIRE].

[26] A.R. Frey and J. Roberts, The Dimensional Reduction and Kähler Metric of Forms In Flux and Warping, JHEP 10 (2013) 021 [arXiv: 1308.0323] [INSPIRE].

[27] H.-Y. Chen, Y. Nakayama and G. Shiu, On D3-brane Dynamics at Strong Warping, Int. J. Mod. Phys. A 25 (2010) 2493 [arXiv:0905.4463] [InSPIRE].

[28] P.A.M. Dirac, The Theory of magnetic poles, Phys. Rev. 74 (1948) 817 [InSPIRE].

[29] B. Cownden and A.R. Frey, Dirac Branes, work in progress.

[30] A. Sen, Covariant Action for Type IIB Supergravity, JHEP 07 (2016) 017 [arXiv: 1511.08220] [INSPIRE].

[31] J.H. Schwarz, Covariant Field Equations of Chiral $N=2 D=10$ Supergravity, Nucl. Phys. B 226 (1983) 269 [INSPIRE].

[32] D. Baumann, A. Dymarsky, I.R. Klebanov and L. McAllister, Towards an Explicit Model of D-brane Inflation, JCAP 01 (2008) 024 [arXiv:0706.0360] [INSPIRE].

[33] S. Kachru, J. Pearson and H.L. Verlinde, Brane/flux annihilation and the string dual of a nonsupersymmetric field theory, JHEP 06 (2002) 021 [hep-th/0112197] [INSPIRE].

[34] J. Polchinski, String theory. Vol. 2: Superstring theory and beyond, Cambridge University Press, Cambridge U.K. (2007).

[35] E. Poisson, A. Pound and I. Vega, The Motion of point particles in curved spacetime, Living Rev. Rel. 14 (2011) 7 [arXiv: 1102.0529] [INSPIRE]. 